SIMPL-1:

SIMULATION IMPLEMENTING MACHINE PROGRAMMING LANGUAGE-VERSION 1

october 1968

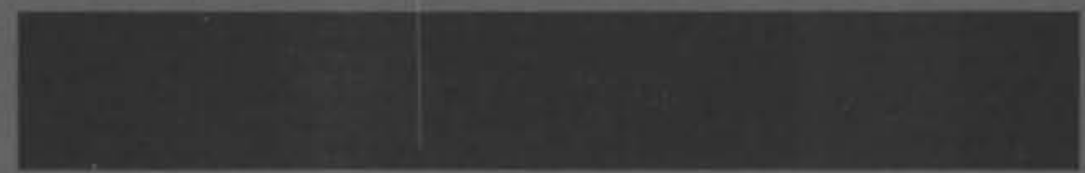

AEC RESEARCH \& DEVELOPMENT REPORT 


\section{LEGAL NOTICE}

This repont was prepaied at an occount of Government spansored work. Nelthes the United States nor the Commission, nor any pesan peting on bahalf of the Commistion:

A. Makes ony waikanty of represenfation, expressed of implied, with respect to the urcuracy, com. pleteness, or usefulnets of the information contained in this, teport, of that the use at any information, apporatus, method, or process diclosed in this report may not infringe privotely owned rights; or

8. Assurtes any fiabilities with respect to the use of, of for damoget resulting froth the use of ony informotion, opporatus, melthad or process divilosed in this report.

As used is the obove. "peison outing on behall of the Commision* Inctudes any employee br controctor of this Commistion, or emplayee of such contractor, to the extant that tuch emplayse or can. tractor of the Commisalan, or eimployee of wich cantractor prepornt, distamioates, ar provides accest to. ony information pussuant to his empleyntent or contract with the Canimisaion of fis employment with juch contracior.

\section{PACIFIC NORTHWEST LABORATORY}

ECHLANG, WASHENGTON

aperated by

BATTELLE MEMORLAL INSTITUTE

for the

UNITEO STATES ATOMIC ENERGY COMMISSION UNDER CONTRACT ATI45-1)-1830 


\author{
BNWL -878 \\ UC-32, Mathematics and Computers
}

\title{
SIMPL-1: SIMULATION IMPLEMENTING MACHINE PROGRAMMING LANGUAGE-VERSION 1
}

\author{
Edited and Assembled By \\ R。D. Benham \\ Control and Instruments Department \\ Systems and Electronics Division
}

\author{
Principal Contributors \\ C. R. Cole \\ P. J. Dionne \\ H. F. Foote \\ L. H. Gerhardstein \\ W. F. Lenzke \\ G. R. Taylor \\ G. A. Worth

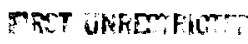

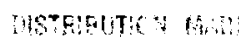 \\ Cit IfFis
}

October 1968

\author{
BATTELLE MEMORIAL INSTITUTE \\ PACIFIC NORTHWEST LABORATORY \\ RICHLAND, WASHINGTON
}


Printed in the United States of America Available from

Clearinghouse for Federal Scientific and Technical Information National Bureau of Standards, U.S. Department of Commerce Springfield, Virginia 22151

Price: Printed Copy $\$ 3.00$; Microfiche $\$ 0.65$ 


\section{SIMPL-1: SIMULATION IMPLEMENTING MACHINE \\ PROGRAMMING LANGUAGE-VERSION 1}

\section{PREFACE}

Two of the most difficult problems impeding the scientific revolution relate to education of computer users and control of inflationary computer costs. Many recognized authorities have publicly stated, "Computerized solutions are costing 100 times too much."

This report does not claim to completely solve both the educational and economics problems facing science and engineering. However, it does point to an area where cost savings of 100-to-1 and time savings of 500-to-1 can be realized. The techniques for realizing such savings are illustrated with tutorial examples of varying complexity from the main report through each appendix. 


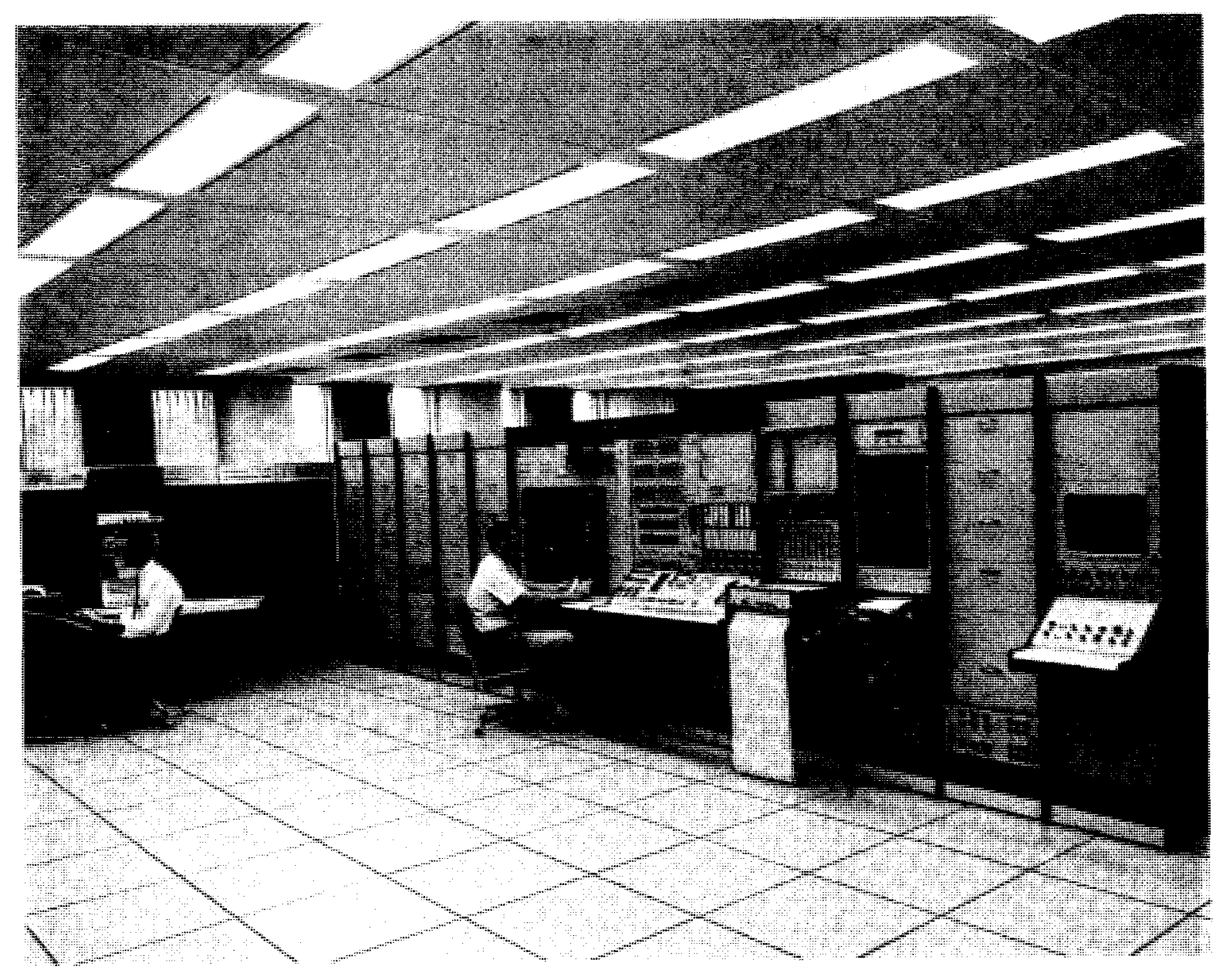

Hybrid Computer System 
SIMPL-1 is a hybrid operating software system for a fixed point PDP-7 digital computer and a Beckman 2133 analog computer. The programming method associated with SIMPL-1 belongs to the same family of continuous simulation language as MIDAS and PACTOLUS. The method is a dialect of the Digital Equipment Corporation's PDP-7 assembly language. When used on our most sophisticated nuclear plant simulation the hybrid processes information about 400 times faster than more conventional programming methods employing a UNIVAC 1108 digital computer equipped with floating point hardware.

When SIMPL-1 programming methods are used without including the analog computer as part of the system, the effective speed of the system is decreased. However, the SIMPL-1 programming method increases the effective computing speed by factors up to 1000 as compared to software floating point FORTRAN.

The digital computer replaces the following analog elements on our largest hybrid simulation:

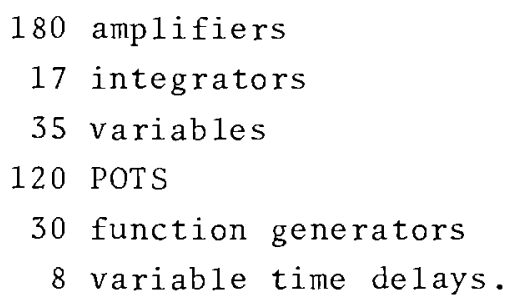

The computing frame time for this simulation is approximately $20 \mathrm{msec}$, which corresponds to processing speeds 10 times faster than real time. 


\section{TABLE OF CONTENTS}

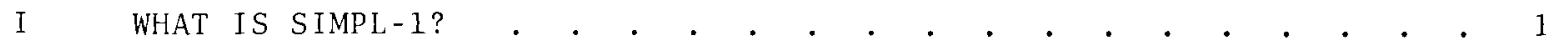

I I HOW DO SIMPL-1 AND FORTRAN COMPARE? . . . . . . . . . . . . . . . 2

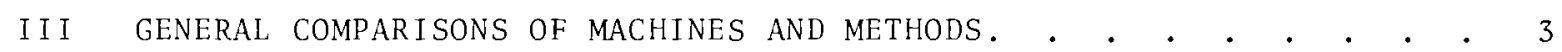

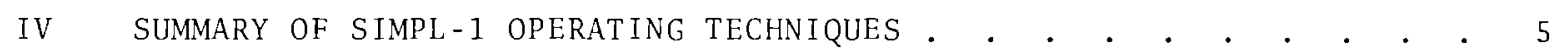

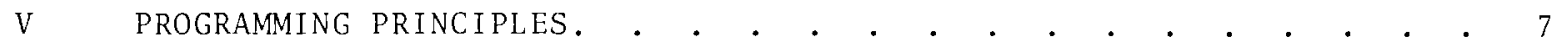

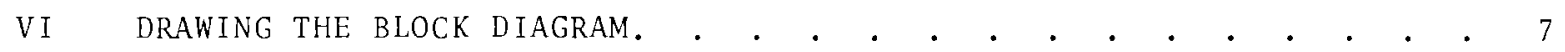

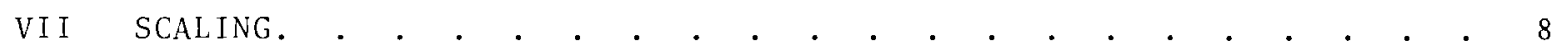

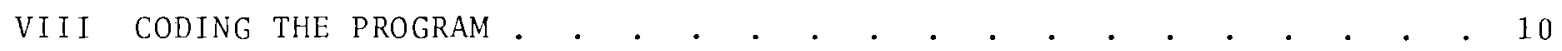

Method for Connecting Building Blocks. . . . . . . . . . . . 10

Main Program Sequence (Without A/D and D/A Conversion) . . . . . 11

$\mathrm{A} / \mathrm{D}$ and $\mathrm{D} / \mathrm{A}$ Conversion. . . . . . . . . . . . . . . . 12

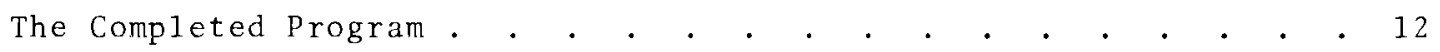

Summary Showing Necessary Elements of SIMPL-1 Programs • • . . $\quad 12$

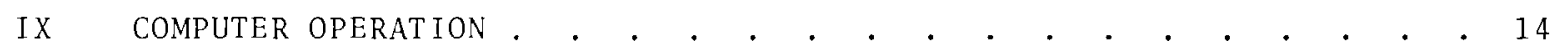

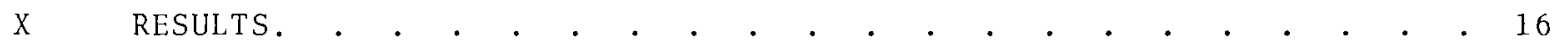

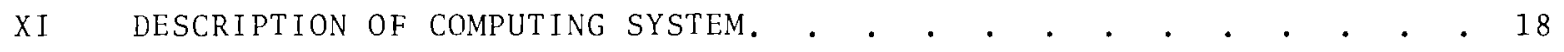

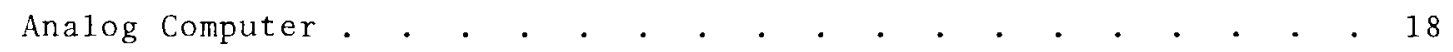

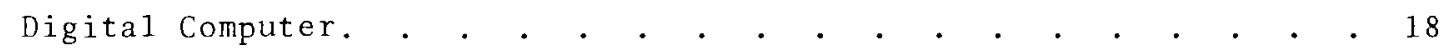

Central Processor Modification . . . . . . . . . . . . . . 19

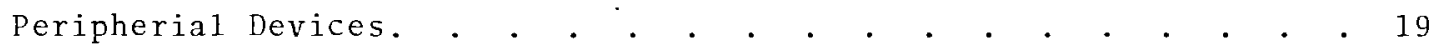

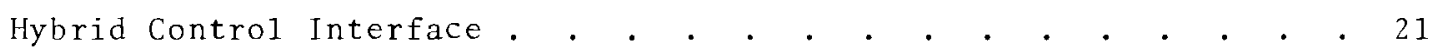

ACKNOWLEDGMENTS • • • • • • • • • • • • • • • • • • • • •

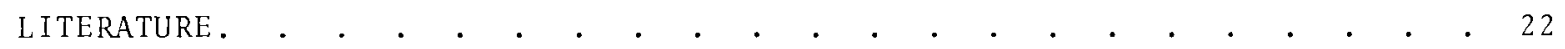

APPENDiX A, SOURCE LANGUAGE SYMBOLS . . . . . . . . . . . . . . . . . . . A- 1

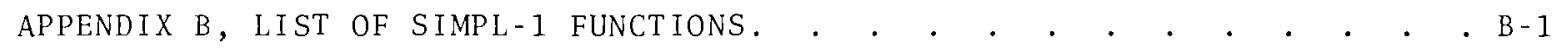

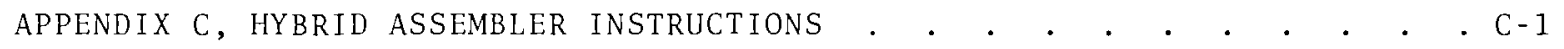

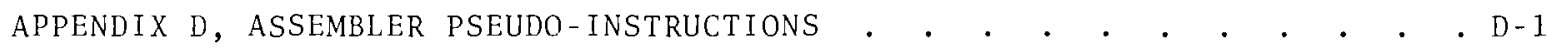

APPENDIX E, AUTOMATIC PRIORITY INTERRUPT INSTRUCTIONS AND EXAMPLE • . . E- .

APPENDIX F, SIMPL-1 SYSTEM PROGRAM INDEX. • . . . . • • . . . . . . . . F-1

APPENDIX G, SIMPL-1 SYSTEMS PROGRAM OPERATING INSTRUCTIONS. . . . . . . G-1

APPENDIX H, TYPICAL PROGRAMMING AND OPERATING ERRORS. . . . . . . . . . H- 1

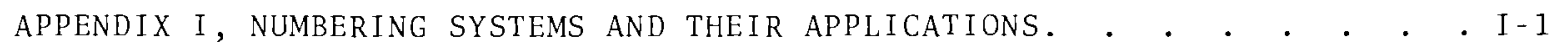

APPENDIX J, INDICATORS AND CONTROLS FOR DIGITAL COMPUTER AND INTERFACE. • J-1

APPENDIX K, EXAMPLE MULTIPLY AND DIVIDE PROGRAMS WITHOUT

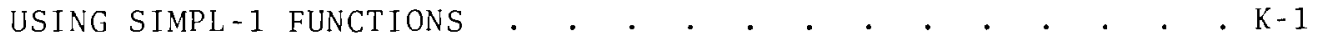

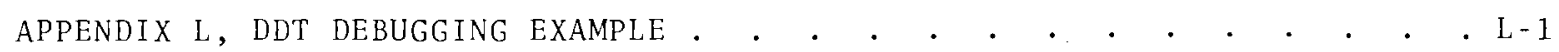

APPENDIX M, SIMPL-1 HYBRID SOLUTION FOR THE PILOT EJECTION STUDY. . . .. M- 1

APPENDIX N, SIMPL- 1 ALL DIGITAL SOLUTION FOR THE PILOT

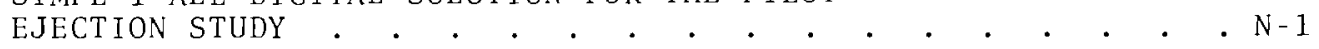

APPENDIX O, PROCESS CONTROL EXAMPLE ILLUSTRATING ASSEMBLY LANGUAGE

PROGRAMMING OF API CLOCK SERVICE ROUTINES . $\quad . \quad \cdot \quad \cdot \quad . \quad . \quad . \quad 0-1$

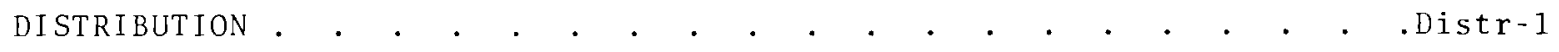


$-$ 


\section{SIMPL-1: SIMULATION IMPLEMENTING MACHINE PROGRAMMING LANGUAGE-VERSION 1}

\section{WHAT IS S SIMPL- I?}

SIMPL- 1 is a hybrid operating software system for a fixed point PDP-7 digital computer and a Beckman 2133 analog computer. It was developed by modifying and augmenting the (Digital Equipment Corporation furnished) basic PDP-7 software system. With the exception of the program library given in Appendix $B$, the 31 different programs that comprise the SIMPL-1 system are categorized (Appendix F) into the following functions: DECTAPE INPUT/OUTPUT, PAPER TAPE INPUT/OUTPUT, CARD INPUT/OUTPUT, EDITOR, ASSEMBLERS, DEBUGGING PROGRAMS, MEMORY DUMPS, and ANALOG SETUP and CHECK OUT.

The programming method associated with SIMPL-1 belongs to the family of continuous simulation languages such as MIDAS ${ }^{(5)}$ and PACTOLUS ${ }^{(8)}$. The acronym SIMPL-1 (Simulation Implementing Machine Programming LanguageVersion 1) was chosen because it is somewhat descriptive of the programming method. That is, it implies that the method is simple to learn and apply, and that a lower level language (i.e., machine language) is necessary. However, the acronym is deceiving because assembly language rather than machine language is used. The programming method used with the SIMPL-1 system is not new. Rather, it is a dialect of the Digital Equipment Corporation's PDP-7 assembly language with rules and symbols added for programming convenience $(: * 1+1 \$$ ) (See Appendix A). Five hardware instructions were also added to the PDP-7, and the teletype interface was modified (See Section XI).

In most hybrid systems the analog is considered a peripheral device to the digital. However, the SIMPL-1 programming methods allow consideration of the digital as peripheral to the analog. Nevertheless, SIMPL-1 programming methods can be used without including the analog computer as part of the system. When used this way, the result is that problem solution times are decreased by factors of 10 to 1000 as compared to software floating point FORTRAN methods, (i.e., greater time decreases are realized as problem complexity increases).

If the SIMPL-l concept is implemented on a fixed point digital computer, the size of the computer is reduced by a factor of two and this should reduce computer cost [according to an indirect application of Grosch's Law (21)] by a factor of four. Furthermore, it opens an area where cost savings of $100-$ to- 1 can be realized.

In the following sections SIMPL-1 and FORTRAN are compared in general. The "Pilot Ejection Study" is then used as a basis to compare the use of SIMPL-1 with other languages on various machines. Programming principles, practices and computer operation are illustrated in the main body of the 
report. Finally, the appendices contain detailed programming and operating instructions as well as more detailed examples.

Further work on implementing simulation methods with assembly language is continuing in areas such as (1) MACRO assembly language, (2) magnetic tape, and (3) drum interacting systems. These variations will be noted by different version numbers (i.e., SIMPL-2, etc.).

\section{HOW DO SIMPL-1 AND FORTRAN COMPARE?}

Most scientific programming for large digital computers is through FORTRAN. The main advantages of FORTRAN are that it is easy to use because it accepts statements which resemble algebraic mathematical formulas, and that the programmer need not concern himself with the manual operation of the machine.

For small to medium size scientific problems, FORTRAN does an adequate job. However, as problems become more complex, memory requirements and computer running time become excessive. Some scientific problems require over $65 \mathrm{~K}$ fast memory locations and from $10 \mathrm{~min}$ to $10 \mathrm{hr}$ of running time to completely produce the desired answers.

When this happens, it becomes necessary to re-evaluate the problemsolving method. In fact, analog and recent hybrid computers have been used to overcome the economic penalties associated with FORTRAN and large expensive digital computers.

Most commercial and privately built hybrid computers use an altered FORTRAN package. This, of course, retains the basic equation-oriented advantages of FORTRAN. Long computer running times are decreased by solving the "high frequency" problems on the analog computer. In the hybrid environment the slowest processor is the digital computer (because the digital is a serial processor and an analog is a parallel processor). Since speed and memory are critical, changes in philosophy that decrease memory requirements and increase processing speed are desirable. (of course, it is also desirable to maintain simple programming methods.)

In this respect it is useful to point out the disadvantages of FORTRAN compilers. First, the transformation of FORTRAN statements to machine language (called compiling) is very inefficient (approximately 33\% for fixedpoint computers). That is, the compiler produces three times more instructions than necessary to do the job, which in effect creates two problems. First, more core memory is required to hold the machine language (compiled) program, and second, more computer time is required to process the program.

High level interpreter programs, such as MIDAS, are even more wasteful of memory and processing time. For example, MIMAC ${ }^{(11)}$ requires $25 \mathrm{~K}$ core locations to hold the interpretive program. However, simulator programs are easier to program than FORTRAN. 
The SIMPL-1 method retains much of the programming simplicity of interpretive languages (like MIDAS) and significantly reduces the memory and computer running requirements to solve a problem (by factors of 5 to 500 ).* A SIMPL-1 program is prepared by interconnecting mathematical building blocks with assembly language.

The relocatable feature of the SIMPL-1 assembler allows an assembled main program to call previously assembled hybrid subroutines through a 1 inking loader. (Absolute hybrid programs are also possible.) By using the features of Hybrid DDT (Digital Debug Tape), (24) modifications to an assembled program can be made on-line without the need for reassembling.

The disadvantages of SIMPL-1 as implemented on the EASE 2133 analog computer (150 + amplifiers) and PDP- 7 digital computer ( $8 \mathrm{~K}$ of 18 bit words) are: are:

(1) The necessity to scale problem variables to overcome the fixed point characteristics of the digital computer. (In general, this is not a severe limitation because scaling is required for the fixed point analog computer.)

(2) The necessity of programmer interaction with the computers.

Because of an unfamiliarity with scaling and machine operating procedures, most FORTRAN-oriented scientists and engineers could regard these as intolerable limitations. However, the economics of solving complex problems have shown that significant cost and time savings (up to 100 to 1 over modern large digital FORTRAN methods) can be realized through hybrid simulation. This should be adequate justification for learning and applying new hybrid techniques to large scientific simulation problems.

\section{I . GENERAL COMPARISONS OF MACHINES AND METHODS}

Very little published work is available on comparing machines and methods for solving scientific problems. It is therefore difficult to select a comparison criterion acceptable to all scientific computer users.

The Pilot Ejection Study used as an example in the MIDAS and MIMIC programming guides is being used by R. D. Brennan (1) of IBM as an industry-wide "bench mark" problem. Others, such as the MONSANTO $(2,3)$ and PHYSBE ${ }^{(4)}$ bench mark problems, require much more computer capability than the Pilot Ejection Study. However, they are not as widely used.

Since the Pilot Ejection Study was the easiest to program and most widely known bench mark problem, it was used to evaluate the relative efficiencies shown in Table 1 for the SIMPL-1 method. (The programs are shown in Appendices $M$ and N.)

* Programs like PACTOLUS(8) and DES-1(7) have been developed for on-line digital simulation. PACTOLUS is at least an order of magnitude slower than DES-1 and SIMPL-1. 


\begin{tabular}{|c|c|c|}
\hline \multicolumn{3}{|c|}{$\begin{array}{l}\text { TABLE 1. Comparison of Speed and Efficiency } \\
\text { for the Pilot Ejection Study }\end{array}$} \\
\hline $\begin{array}{l}\text { Method of } \\
\text { Solving Problem }\end{array}$ & $\begin{array}{l}\text { Minimum Computer Time Required } \\
\text { to Give Equivalent Accuracy, sec }\end{array}$ & $\begin{array}{l}\text { Relative } \\
\text { Efficiency }\end{array}$ \\
\hline $\begin{array}{l}\text { FORTRAN } \\
\text { PDP - } 7 \\
\text { UNIVAC } 1108 \\
\text { MIMIC } \\
\text { IBM } 7090 \\
\text { UNIVAC } 1108 \\
\text { SIMPL-1 (a11 digital) } \\
\text { SIMPL- } 1 \text { (hybrid) } \\
\text { AD/4 analog computer }\end{array}$ & $\begin{array}{l}7 \\
1.4 \\
45 \\
2(a) \\
0.0615 \\
0.043 \\
0.00043\end{array}$ & $\begin{array}{c}1 \\
5 \\
0.155 \\
3.5 \\
110 \\
160 \\
16000\end{array}$ \\
\hline $\begin{array}{l}\text { (a) Neglects } 26 \text { sec } \\
\text { of I/0 time). F } \\
\text { of DTMIN (minimu } \\
\text { of } 0.0001 \text { requir } \\
\text { more seconds tha }\end{array}$ & $\begin{array}{l}\text { input/output time (the IBM } 7090 \text { re } \\
\text { ive parameter runs were made to de } \\
\text { m computing interval) on running t } \\
\text { es one more second than DTMIN of } 0 \\
n \text { DTMIN of } 0.01 \text { sec. }\end{array}$ & $\begin{array}{l}\text { quired } 95 \text { sec } \\
\text { termine effect } \\
\text { ime. A DTMIN } \\
.001 \text { and two }\end{array}$ \\
\hline
\end{tabular}

For this relatively trivial problem SIMPL-1 is from 110 to 160 times more efficient than FORTRAN as implemented on the fixed point PDP-7 computer.

In comparing digital computer speeds it is also necessary to compare accuracies. The PDP-7 FORTRAN computing time* 1 isted in Table 1 utilized a rectangular integration scheme with a $0.01 \mathrm{sec}$ step size. Since the problem on $1 y$ requires $0.43 \mathrm{sec}$ of real-time to run, 43 time steps were taken. The maximum error in any variable was $1.8 \%$. Parameter studies were then made to determine running time as a function of step size and accuracy. To obtain the same accuracy as real-time SIMPL-1 (both hybrid and digital) and the predictor-corrector integration methods used by MIMIC, a step size of 0.0013 sec and a computer time of $52 \mathrm{sec}$ are required. The time required to process $0.43 \mathrm{sec}$ of real-time with FORTRAN on the fixed point PDP-7 computer is:

$$
T=\frac{0.0767}{(\text { Step Size }) 0.98}
$$

When FORTRAN was used on a UNIVAC 1108 (with floating point hardware) the problem required about $6 \mathrm{sec}$ to compile and from 1.4 to $3 \mathrm{sec}$ (depending on step size) to execute.

In ten times real-time, the hybrid method produced 41 updates, which is roughly equivalent to the 43 steps produced by FORTRAN with 0.01 sec time steps. Twenty percent more hybrid speed could have been realized by individually programming analog-to-digital and digital-to-analog conversion. In ten

* It took over 15 min to compile 
times real-time the al1 digital SIMPL-1 method produced 29 time steps and was in error by $7.5 \%$. By ratio, 44 time steps could be accommodated in about 7 times real-time to produce answers with a maximum error of $1.8 \%$, Most values were within $0.5 \%$, but in real-time, the overal1 accuracy is within $0.2 \%$.

The FORTRAN memory requirements were not determined. However, the PDP-7 assembled FORTRAN compilation generated 3.3 times the number of instructions required by the hybrid SIMPL-1 program and 1.5 times the number required by the SIMPL-1 all digital program. The hybrid SIMPL-1 problem required $9.3 \%$, and the SIMPL-1 all digital problem required $23.3 \%$ of the digital computers 8192 word memory. If the output variables are directly plotted (on a scope, $X-Y$ plotter, or strip chart recorder), the memory requirements of the all digital problem are reduced by $7.6 \%$

To the novice, the detailed analysis of computing times given above may appear to be an unnecessary exercise. For a problem like the Pilot Ejection Study there is little gained in speeding up the problem solutions (even above the $52 \mathrm{sec}$ required by FORTRAN when using an 0.001 time step).

From the above speed comparisons the relative speed advantages of SIMPL-1 are demonstrated. This speed advantage is realized even for nontrivial problems which require $10 \mathrm{~min}$ to $10 \mathrm{hr}$ of large computer processing times. SIMPL-1 offers speeds advantages up to 500 over al1 digital FORTRAN methods (implemented on machines with floating point hardware).

To the experienced hybrid programmer it may appear that an attempt was made to compare "apples and oranges" by comparing software floating point with hardware floating point methods. Also, no attempt was made to estimate the necessary planning and educational costs for implementing the SIMPL-1 concept on a larger scale (i.e., company-wide basis). Criticism of this nature is indicative of the need for additional $R$ \& $D$ on computer utilization.

Since such amazing speeds can be realized through hybrid techniques, there is ample justification for wider use of hybrid problem solving methods, especia11y when large multimiliion dollar digital computers are made obsolete every 3 or 4 years by merely increasing the hardware speeds by factors of 2 to 10 .

\section{SUMMARY OF SIMPL-I OPERATING TECHNIQUES}

Figure 1 summarizes the steps required to solve a hybrid computer program. It is generally recommended that programs first be punched on cards for editing convenience and more effective computer utilization. However, the computer's 


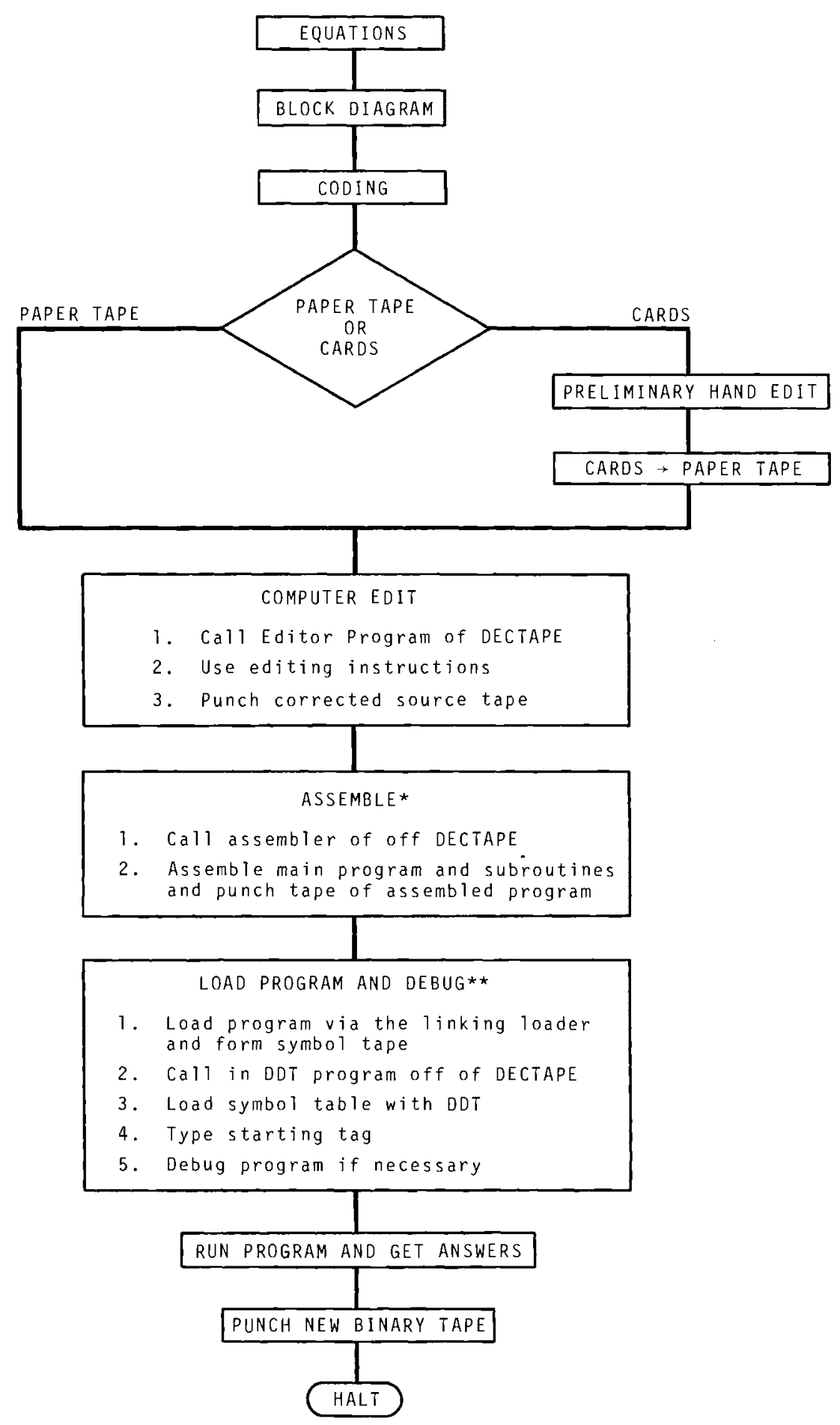

* See Appendix $G$, page 5

* See Appendix G, page 6 
editor program is usually required, especially for nonstandard key punch characters, such as a backward slash (\).

The typical computer operating instructions shown in the blocks describing the EDITOR, ASSEMBLER, and DDT are for illustrations only. Conditions of programs in-core will necessitate variations from the given operating instructions. Finally, as a programmer becomes more proficient, he will discover and master specialized techniques for increasing his effectiveness.

\section{PROGRAMMING PRINCIPLES}

A SIMPL-1 program (1ike an analog computer) is composed of numerous computing blocks with inputs and outputs. The building blocks (or operational elements) are listed in Appendix B. Each element has the following characteristics:

(1) A symbol for use in block diagrams. This can nerely be a box with the mathematical symbol included.

(2) A calling sequence. Generally one of the inputs must be loaded in the accumulator before the subroutine is called with a JMS instruction. (Other descriptive information is included in the remarks column of Appendix B.)

(3) An output in terms of the inputs. The output is generally left in the accumulator following the last calling sequence instruction.

\section{DRAWING THE BLOCK DIAGRAM}

The equations to be solved determine the method of ordering the building blocks. For example, consider the system described by the following differential equations.

$$
\begin{aligned}
& \frac{d V}{d t}+K V^{2}+g \sin \theta=0 \\
& \frac{d \theta}{d t}+g \frac{\cos \theta}{V}=0
\end{aligned}
$$

The block diagram (see page 10) can be drawn by utilizing the principle of repeated integration.

(1) Solve equations for highest order derivatives

$$
\begin{aligned}
& \frac{d V}{d t}=-K V^{2}-g \sin \theta \\
& \frac{d \theta}{d t}=-g \frac{\cos \theta}{V}
\end{aligned}
$$


(2) Assume that the highest order derivative for each equation is available as the output of an operational element and integrate successively until each of the lower derivatives and the functions themselves are available. (Note analog integrators invert the sign.)

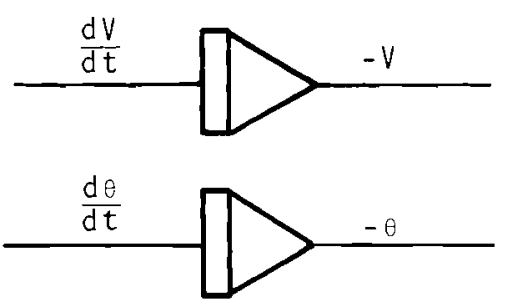

(3) Note that the values for forming the highest order derivatives are now available as the output of operational elements.

(4) Form as the output of computing elements the highest derivatives by using additional computing elements as necessary.

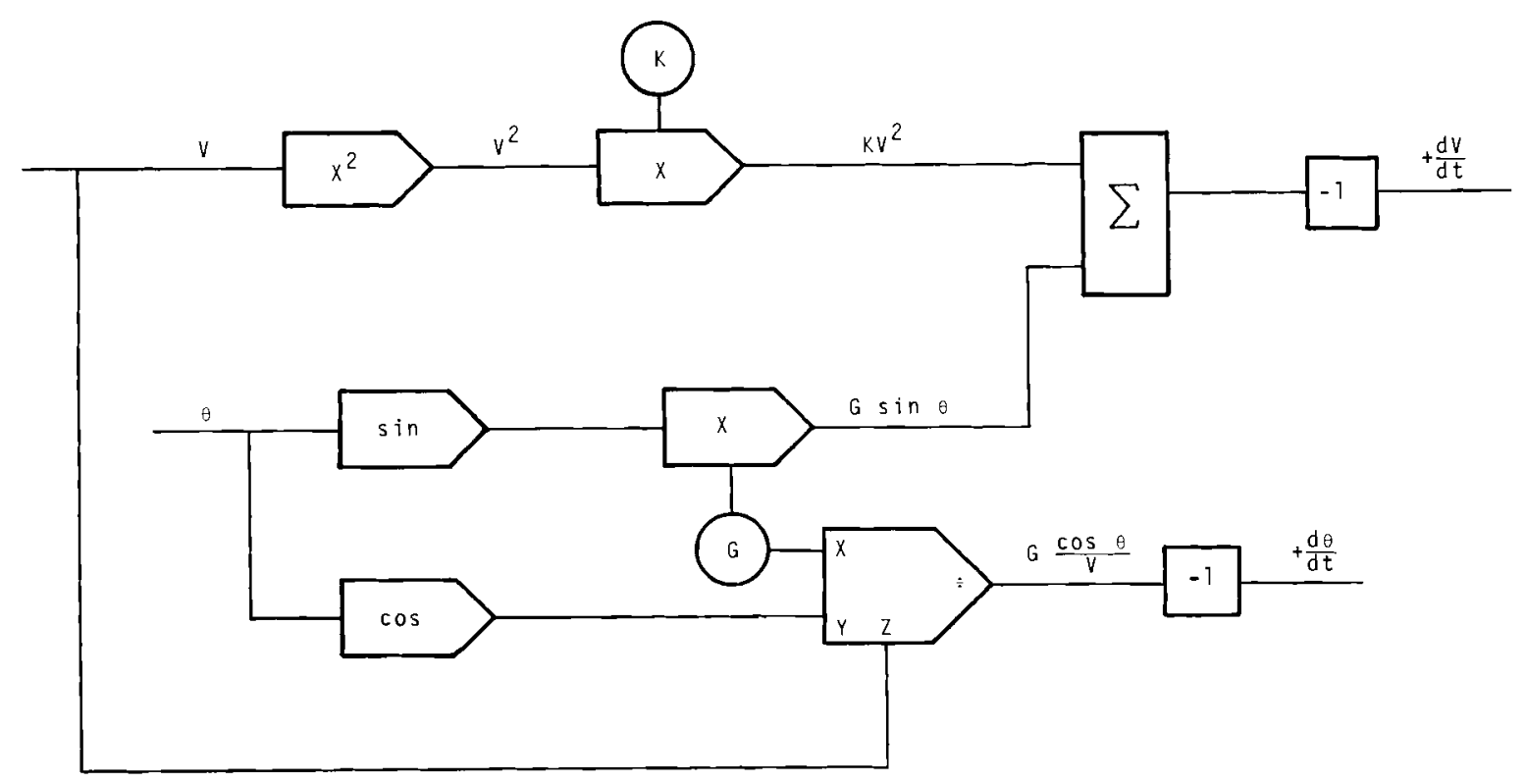

The final block diagram is formed by connecting the units in (2) and (4) above together with digital-to-analog and analog-to-digital conversion trunks after allowance is made for sign reversal of the analog components.

VII. SCALING

Since both the analog and digital computers used in the hybrid system are fixed point machines it is necessary to amplitude scale the equations. (Time scaling may also be necessary, which usually involves changing the gains on all integrators by the same factor.) All analog numbers range between \pm 100 , and all variables transmitted to and from the digital computer must range between \pm 99.99 . However, once a number is in the digital computer, it can range between \pm 1599.99 . 
Analog computing variables should be scaled for operating in a range above $10 \mathrm{~V}$. Digital variables, on the other hand, give better results if their magnitudes are above $50 \mathrm{~V}$. If warranted, digital programs like MIDAS (5) and $\operatorname{APACHE}^{(6)}$ can be used to obtain preliminary scaling information.

The derivative calculations for $\mathrm{dV} / \mathrm{dt}$ and $\mathrm{d} \theta / \mathrm{dt}$ will illustrate the scaling technique.

$$
\begin{aligned}
& \frac{d V}{d t}=-K V^{2}-g \sin \theta \\
& \frac{d \theta}{d t}=-g \frac{\cos \theta}{V}
\end{aligned}
$$

where

$$
\begin{aligned}
K & =1.699 \times 10^{-3} \\
V(0) & \simeq 900 \\
\theta(0) & \simeq 0.043 \text { (radians) }
\end{aligned}
$$

It is clear that $K$ and $\theta$ are too small and $V$ is too large. Since $V$ and $\theta$ are analog variables, scaling factors of 0.1 and 1000 seem reasonable.

That is

$$
\begin{aligned}
0.1 \mathrm{~V} & =90 \\
1000 \theta & =43
\end{aligned}
$$

Since $K$ is entirely a digital variable, a scale factor of $10^{5}$ is reasonable, or

$$
10^{5} \mathrm{~K}=169.9
$$

The scaled block diagrams for the derivative calculations are:

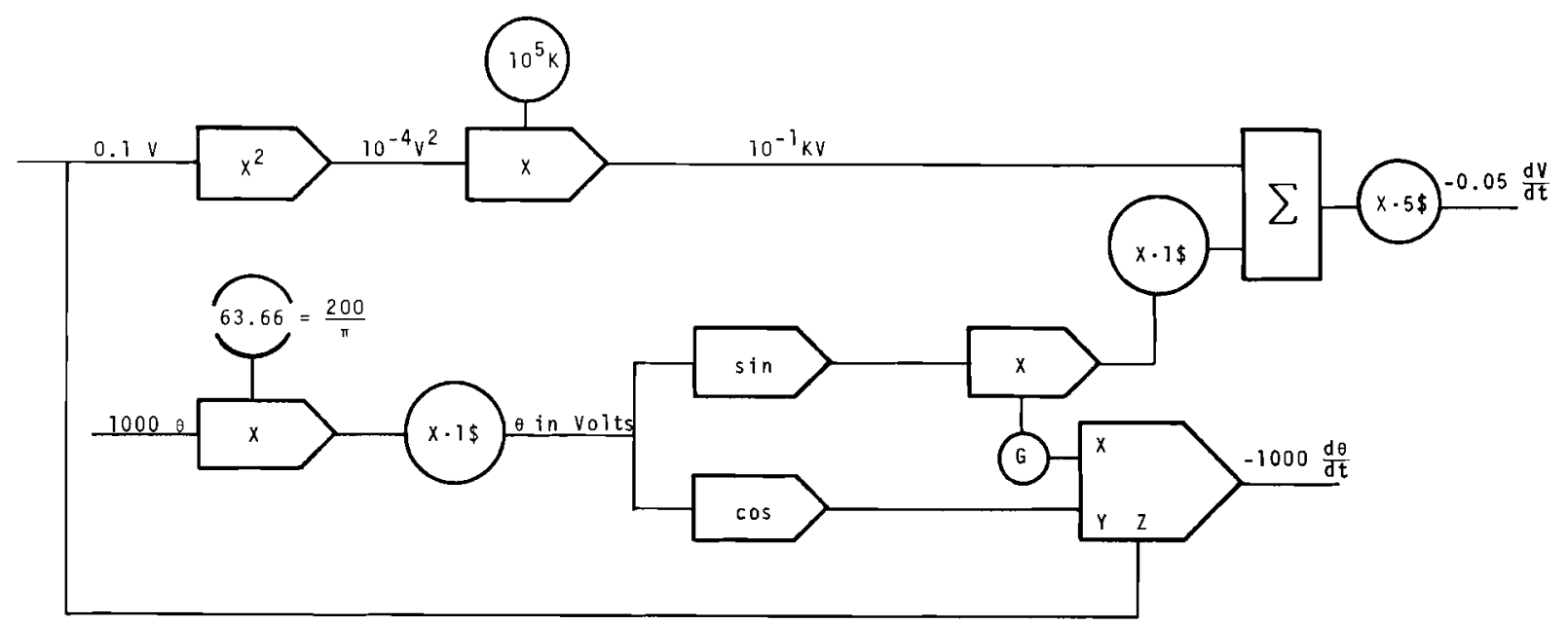

The scale factors written above take into account the division by 100 of SIMPL-1 multiply routines. A scale factor of 0.05 was chosen for $\frac{\mathrm{dV}}{\mathrm{d} t}$ because $0.05 \frac{\mathrm{dV}}{\mathrm{dt}} \simeq 61.0$. Since a scale factor of 0.1 was chosen for $V$ on the analog 
computer, the gain on the analog integrator must be 2 . The scale factor for $\theta$ was chosen to be $1000\left(1000 \frac{\mathrm{d} \theta}{\mathrm{dt}} \simeq 43.0\right)$.

The completed scaled block diagram for this system of equations is:

$0.1 \vee$

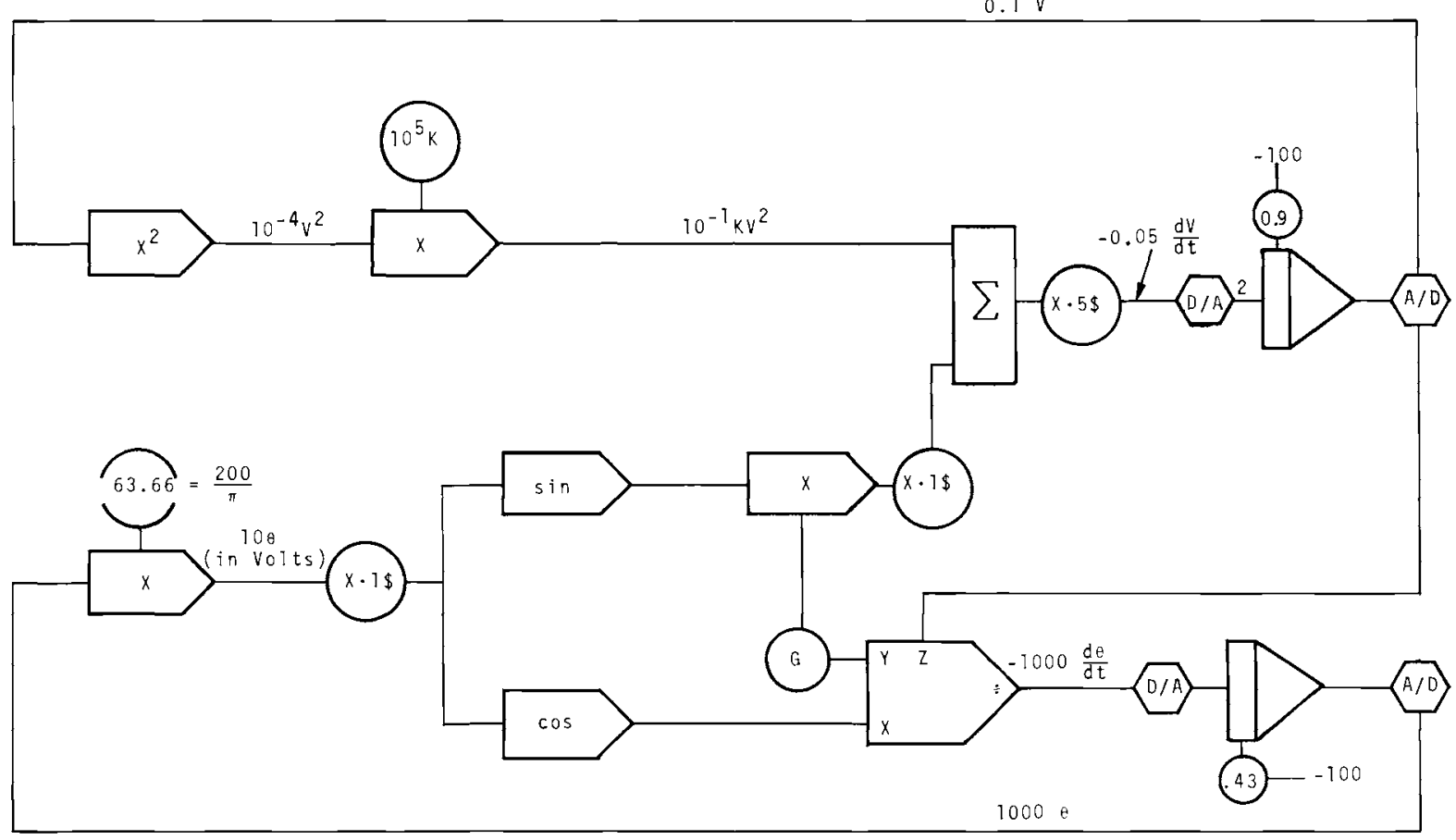

VIII. CODING THE PROGRAM

Once the scaled block diagram is completed the problem is ready for coding. (See Appendix $A$ and Appendix $B$ for explanation of SIMPL-1 and assembly language coding.) Like MIDAS programming (or analog computer programming) coding is accomplished by interconnecting the computing elements. This procedure is explained by: (1) showing how individual blocks are connected, (2) showing the program sequence for the main program loop, (3) showing the analog-todigital and digital-to-analog conversion program sequence, (4) showing the completed program, and (5) summarizing the necessary elements of every SIMPL-1 program.

\section{METHOD FOR CONNECTING BUILDING BLOCKS}

The term $10^{-1} \mathrm{KV}^{2}$ shown in the block diagram is generated with the following coding sequence:
LAC V
JMS HSQ\$
JMS HMPY $2 \$$
LAC K
DAC SAVE1\#

The first instruction (LAC V) loads the accumulator with the value of $V$. The second instruction directs the value of $V$ left in the accumulator to a 
squaring subroutine (HSQ $\$$ ). The answer $\left(10^{-4} \mathrm{~V}^{2}\right)$ from this subroutine is left in the accumulator for multiplication by the constant $K$ with the following sequence:

JMS HMPY $2 \$$

LAC K

A cursory comparison of this sequence with the calling sequence given in Appendix B shows that a LAC instruction (preceding the JMS HMPY 2 ) is missing. However, it is not necessary because the value to be multiplied $\left(10^{-4} \mathrm{~V}^{2}\right)$ is already in the accumulator. After the multiplication, it is necessary to store the value of $10^{-1} \mathrm{KV}^{2}$ in location SAVEl because all inputs to a subroutine must be defined before it is used. The outputs of integrators are defined through the initial conditions.

MAIN PROGRAM SEQUENCE (Without $A / D$ and $D / A$ Conversion)

The method of interconnecting subroutines is repeated, as shown below, until both $-0.05 \frac{\mathrm{dV}}{\mathrm{d} t}$ and $-1000 \frac{\mathrm{d} \theta}{\mathrm{d} t}$ are evaluated.

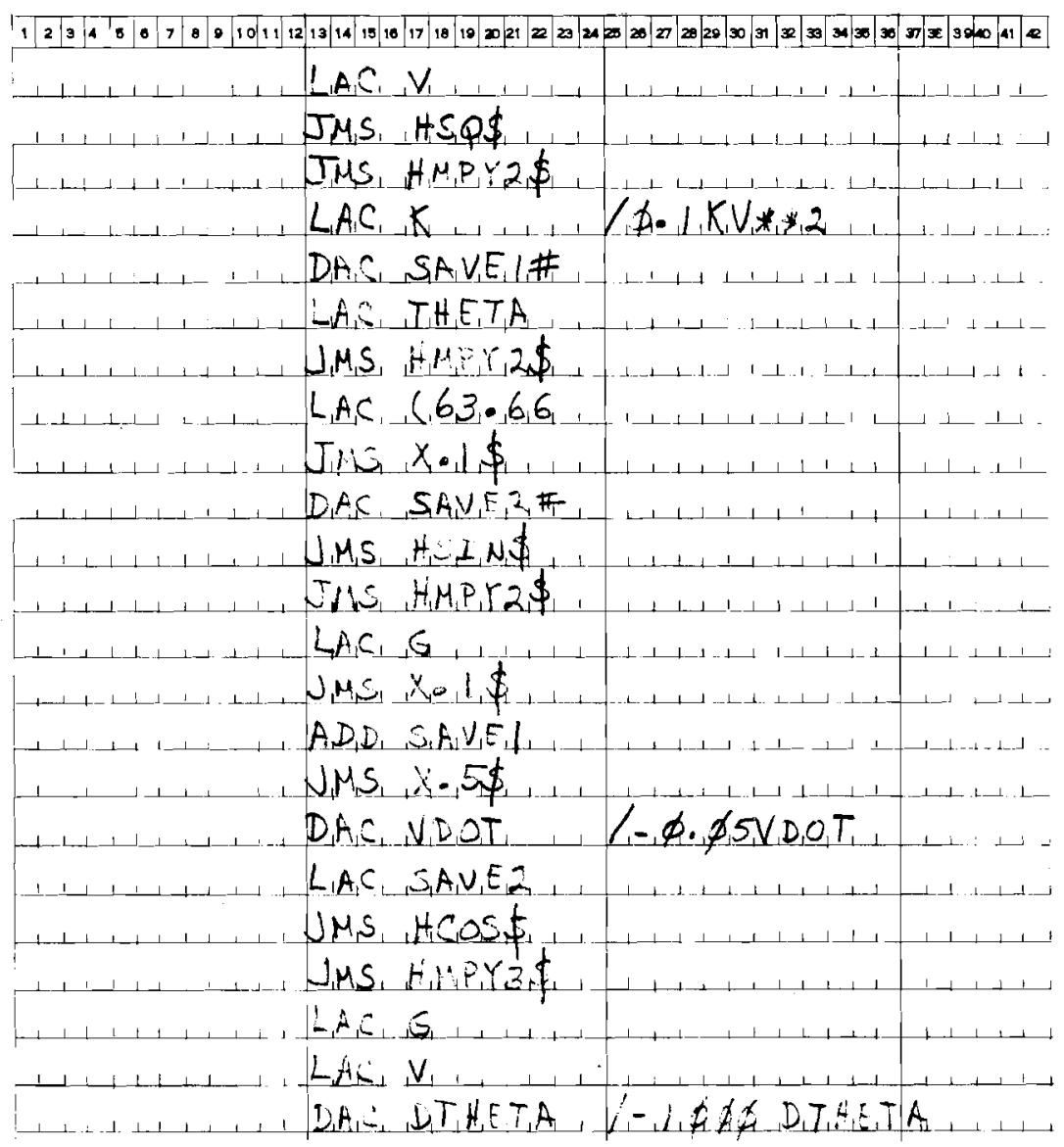




\section{A/D AND D/A CONVERSION}

The analog-to-digital transfer of $V$ and $\theta$ is accomplished with the following sequence:

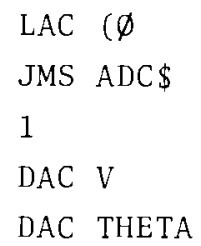

The digital-to-analog transfer for $-0.05 \frac{\mathrm{d} V}{\mathrm{dt}}$ and $-1000 \frac{\mathrm{d} \theta}{\mathrm{d} t}$ is accomplished by:

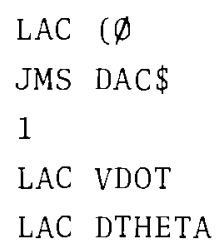

\section{THE COMPLETED PROGRAM}

The program coding for this example can be completed as shown in Figure 2 by adding a title, inserting program constants, initializing the computer, performing a computing termination check, and adding JMP BGN, and a PAUSE statement at the end of the LISTING.

\section{SUMMARY SHOWING NECESSARY ELEMENTS OF SIMPL- 1 PROGRAMS}

Every program should have the following sections:

(1) A title

(2) A program constants section which includes
(a) Storage of program constants (such as $G$ and $K$ )
(b) Function generator storage (if required)
(c) Time delay table storage (if required)
(d) Instructions for automatic priority interrupt channe1s
(e) Teletype header messages

(3) Computer initialization for
(a) Modified instruction set (MIST)
(b) Clearing software flags
(c) Setting up teletype subroutines
(d) etc.

(4) CALCULATION of analog computer initial conditions (if required)

(5) Main program 1oop
(a) Analog to digital conversion
(b) Interconnecting of mathematical building blocks
(c) Digital-to-analog transfer
(d) End of compute test (if required)
(e) Jump to starting address of main computing loop 


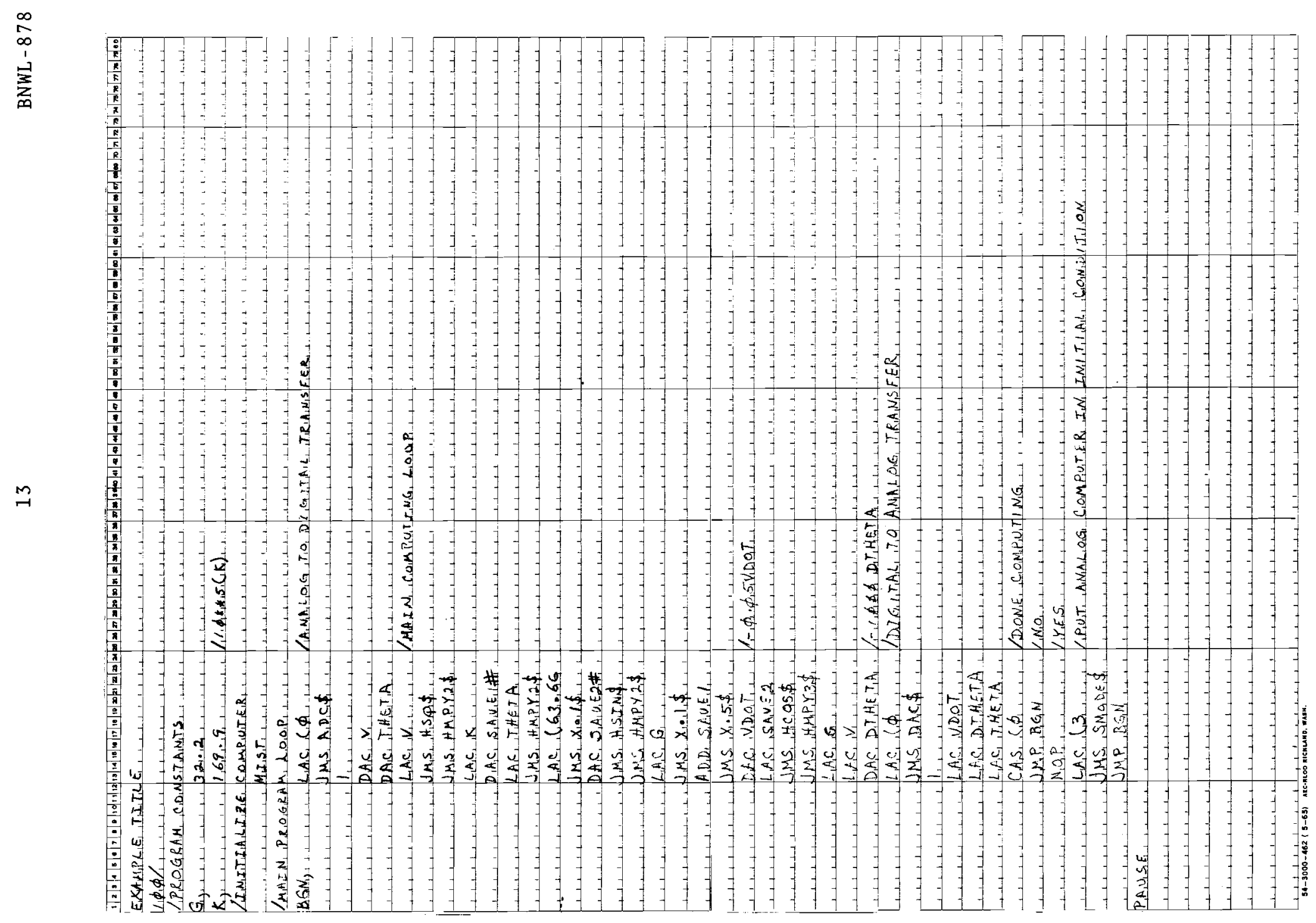

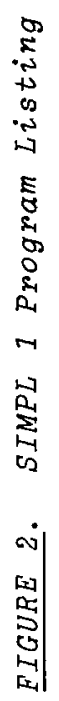


(6) PAUSE

By comparing the summary given above with the listing in Figure 2 , it is obvious that:

(1) The title is: EXAMPLE TITLE

(2) The program constants are $G$ and $K$ (no other constants are necessary)

(3) The only computer initialization required is to select the modified instruction set with the instruction MIST

(4) Calculation of analog initial conditions are not required

(5) The starting address of the main programming loop is BGN. The main computing loop contains all essential elements which include changing the analog computer mode to initial conditions when computing is terminated.

(6) It has a PAUSE statement

\section{COMPUTER OPERATION}

For convenience, the program was first keypunched on cards for hand editing. (Two or three errors were found and corrected.) A paper tape of the program was then obtained by using the PUNCH DECK program (see SIMPL-1 System Program Index, Appendix F). After calling the EDITOR into core memory from DECTAPE, the paper tape source program was read in for final editing and 1 isting. The first block in Figure 3 shows the editor's program listing. The A and comment "append the following to the editor" is included to show what the listing would look like if the program were typed directly into the EDITOR. The $N$ typed at the end of the program is a punch and paging command to the EDITOR.

Normal assembler operating instructions were then followed to obtain a binary (actually called funny format) assembled tape. Since \# symbols were not used in the program for defining DTHETA, THETA, $V$, and VDOT, an error message was printed. The assembler automatically corrected the error and proceeded with the assembly. However, if other errors had been made, additional diagnostics would have been printed. Following the error diagnostics is an alphabetic and numberic listing of tags and subroutines. (Note SIMPL-1 library routines are listed without address specification.) Addresses for library routines are assigned by the linking loader as indicated in the next block. A symbol table for running the program under control of DDT is also punched at this time. A minus sign (-) typed out with the DDT symbol table indicates a missing subroutine.

The symbol table tape was read in by typing TABLE\$. After that the teletype responded with the octal numbers 743 and 13503. (The space between 743 and 13503 is unused core.) 


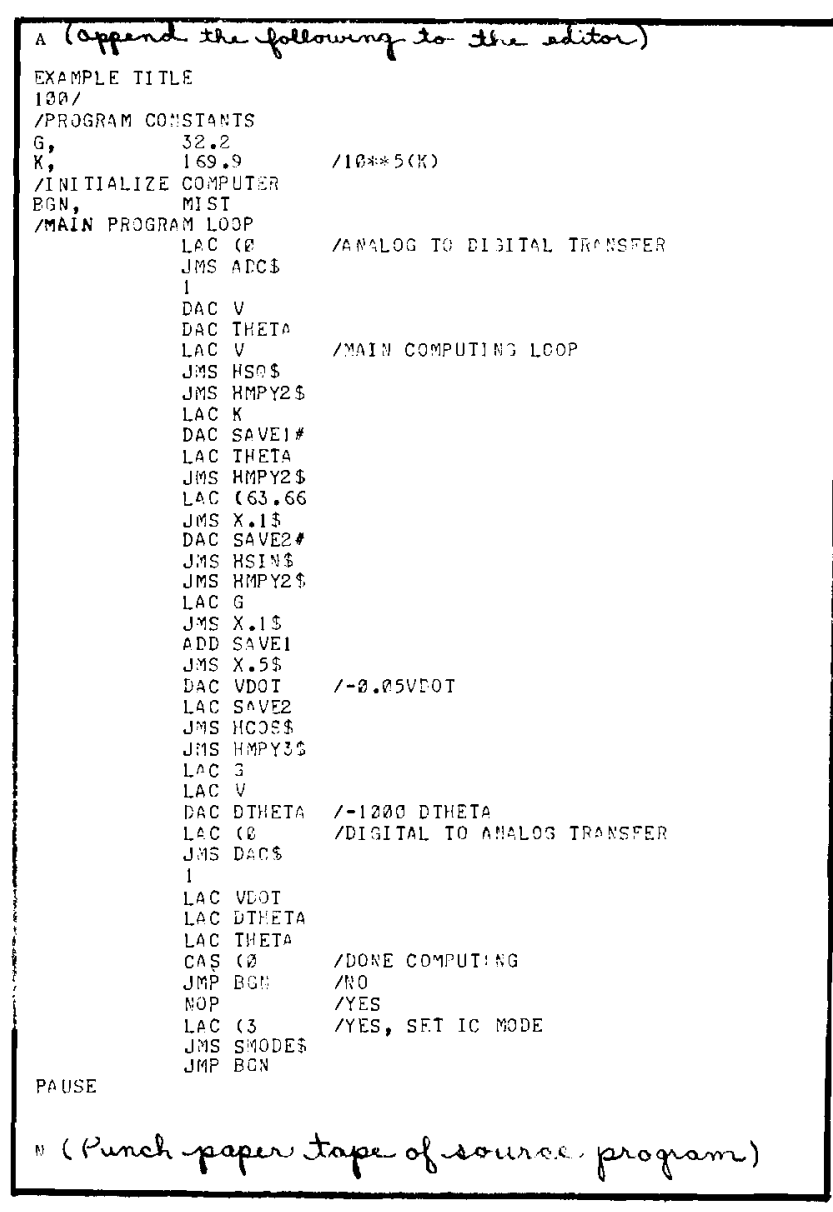

(normal assembles operating inatructione
were followed)

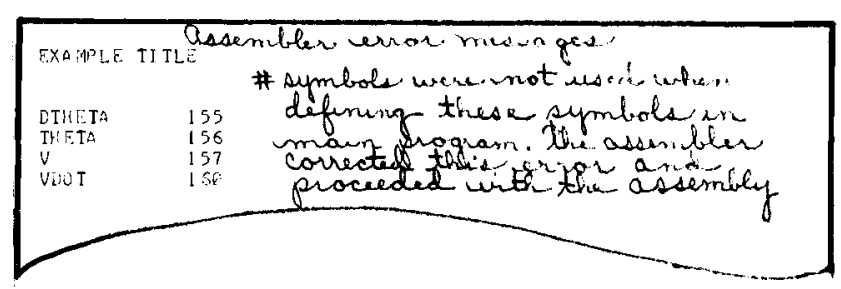

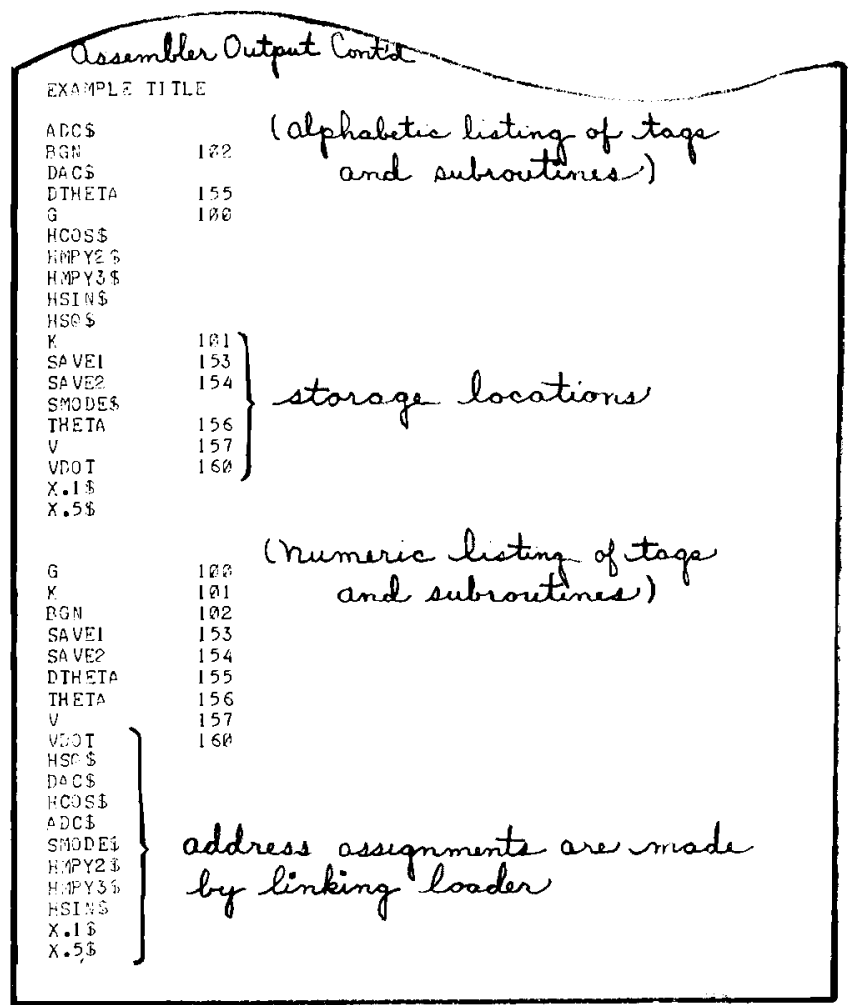

normal instructions fors loaden. relocatable prongra.... through hinking loader a a d running prograw. under continl of DDT were followe.

\begin{tabular}{|c|c|c|}
\hline 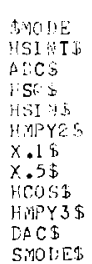 & $\begin{array}{l}742 \\
439 \\
531 \\
265 \\
356 \\
157 \\
256 \\
247 \\
312 \\
220 \\
651 \\
475\end{array}$ & $\begin{array}{l}\text { linkeng loader } \\
\text { a minus sign(-) typod ont } \\
\text { wita listing indicates a } \\
\text { mising }\end{array}$ \\
\hline
\end{tabular}
TAELES Called DDT and read in symbol tope
next open addreses 13503

EG:!

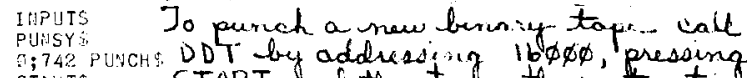
START, and the ri typing the inatructionl 
The next instruction typed was the starting program TAG' (BGN'). Since the program ran, no debugging with DDT was necessary. (Appendix L gives an example of how to debug with DDT and Appendix H lists typical operating and programming errors.)

The last block of Figure 3 shows the instruction sequence for obtaining a single assembled funny format tape. This is an especially useful feature of DDT because funny format patches to the assembled program can be made without reassembling the entire program. The disadvantage is that a new typed source program 1 isting is not available.

Of secondary importance is the ease in reloading the program. The single tape is loaded by calling DDT, typing DEBUG\$, and the starting TAG' (BGN'). If a tape had not been punched, the library would have to be reloaded through the linking loader and the DDT symbol tape reloaded.

\section{RESULTS}

Figure 4 is a strip chart recording of $V$ and THETA versus TIME. For comparison, the same problem was simulated using FORTRAN. Figure 5 shows the FORTRAN statements, the compiled assembly language, and three strip chart recordings.

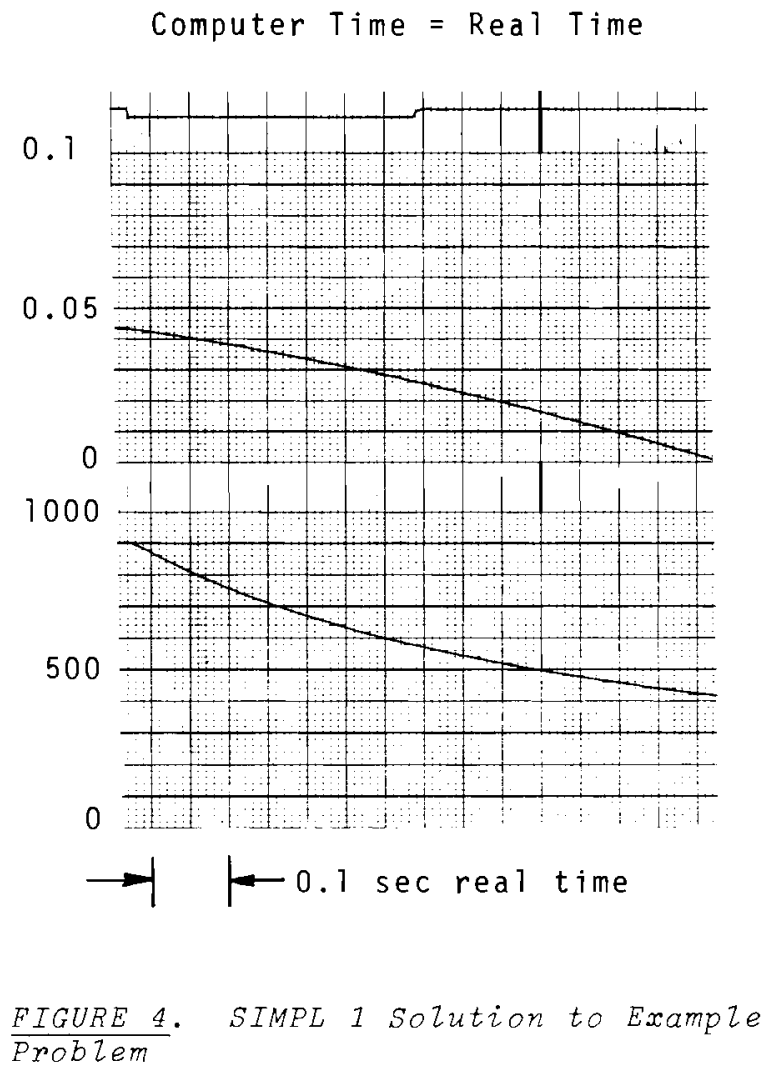




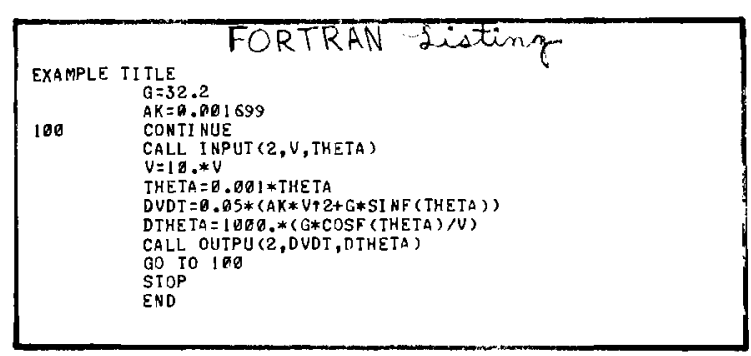

\begin{tabular}{|c|c|}
\hline \multicolumn{2}{|c|}{ EXAMPLE TI ILE FORTRAN Ossemblad Listing } \\
\hline DECIMA & $\begin{array}{l}\text { FIODEC } \\
\text { EXTERNAL } .101, .102, .103, .104, .105, .106, .107, .109, .109 \\
\text { EXTERNAL .1057A,.10DEC }\end{array}$ \\
\hline & $\begin{array}{l}\text { CALST } \\
\text { EXIERNAL EARITH } \\
\text { JMS EARI IH } \\
\text { EFM } \\
\text { LAC .ADA } \\
\text { DAC \$G. }\end{array}$ \\
\hline & $\begin{array}{l}\text { LAC } \text { SAAB } \\
\text { DAC } \$ \text { SAX. }\end{array}$ \\
\hline \multirow[t]{7}{*}{.198} & \\
\hline & EXTERNAL INPUT. \\
\hline & $\begin{array}{ll}\text { CAL I INPUT. } \\
\text { ARX } & (2 \\
A R F & \$ V . \\
\text { ARF } & \text { \$THETA. }\end{array}$ \\
\hline & EFM \\
\hline & $\begin{array}{l}\text { LAC } \dot{V}^{A A C} \\
\text { FMP }\end{array}$ \\
\hline & DAC \$V. \\
\hline & $\begin{array}{l}\text { LAC } \text { FAD } \\
\text { FMP } \\
\text { DACETA } \\
\text { STHETA }\end{array}$ \\
\hline
\end{tabular}

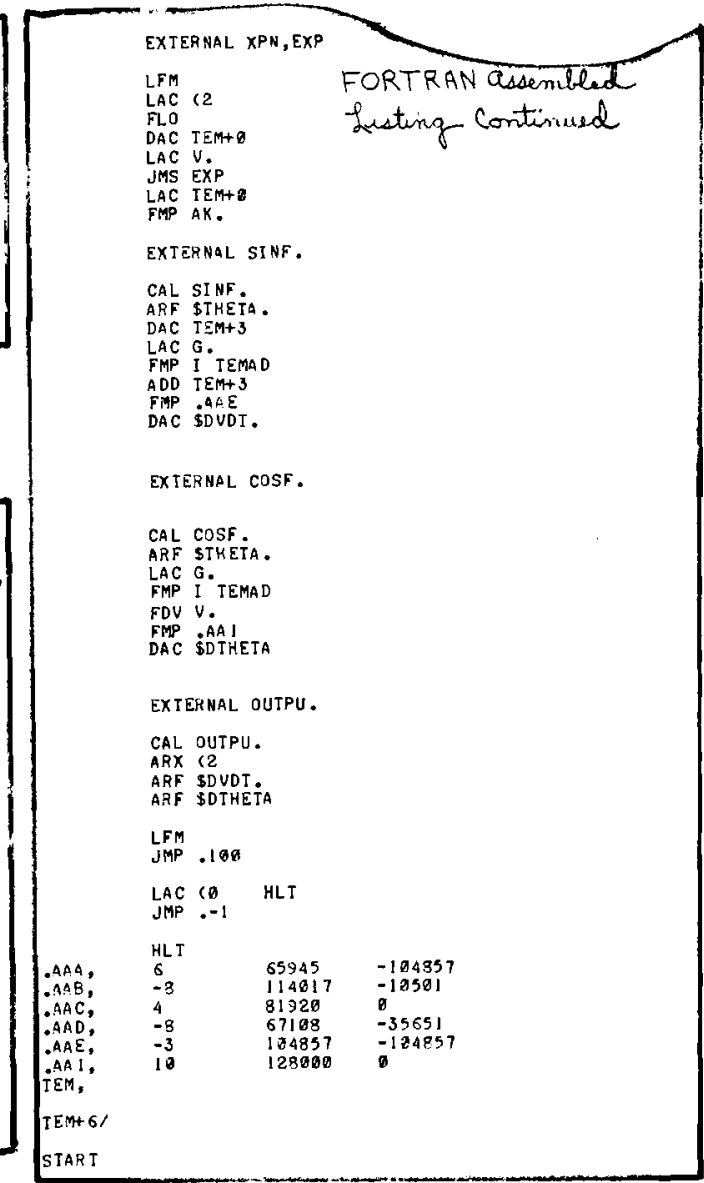

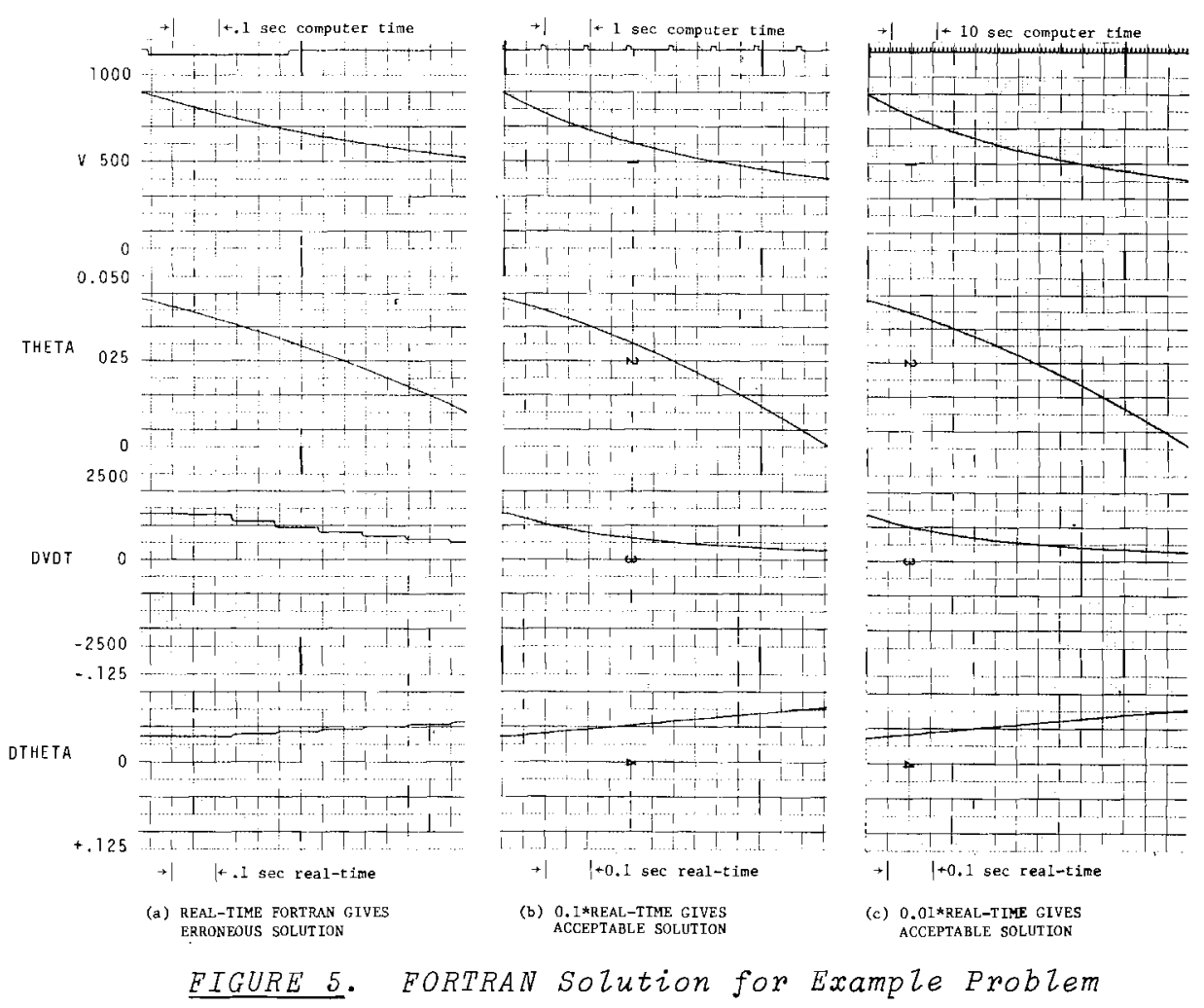


The first strip chart shows the results obtained by solving the problem in real-time (Analog Integrators). Since the FORTRAN frame time was only 0.1 sec the solution did not converge. The second figure shows the result for 0.1 sec real-time. The solution converged, but it required 10 times as much time as the SIMPL-1 program, and the stair-step for DVDT is noticeable. When solving the problem in 0.01 times real-time, the solution converges without stairstepping of DVDT. The conclusions are that the FORTRAN on a fixed point machine is slow. For this particular example, it is 10 times slower than the SIMPL-1 method.

Additional examples of varying complexity are explained in Appendices $L$ through 0 .

\section{DESCRIPTION OF COMPUTING SYSTEM}

The PNL Hybrid Computing System (see References 22 through 35) consists of a Beckman Mode1 2133 analog computer, a Digital Equipment Corporation PDP-7 general purpose digital computer, Adage Inc. data conversion equipment, and the associated control interface. The system provides complete hybrid capability for both data and control.

\section{ANALOG COMPUTER}

The Beckman Mode 12133 analog computer contains 156 amplifiers ( 72 are integrators), 32 multipliers, and 16 function generators. It has 240 servoset pots that utilize the DO/IT system. This computer contains two iterative mode controllers (IDACAS). The Mode 12133 may be coupled to a Beckman Model 1132 analog computer for added hardware capability to solve large problems. Peripheral devices for the Model 2133 analog computer include an X-Y plotter, a Brush eight-channel strip-chart recorder, a cathode-ray tube display, a Friden flexowriter, a Tektronix 564 storage oscilloscope, and a digital logic system. (32)

The digital logic system extends the capability of the analog computer by adding up-down counters, preset counters, buffer and shift registers, gates, analog comparators, and analog switches. The digital logic provides program interrupt signals that are dependent on the value (voltage) of an analog variable. It also provides analog computer "flags" that will initiate, upon flag searching, a digital computer program branch.

\section{DIGITAL COMPUTER}

The PDP-7 is a binary, fixed point digital computer containing an 8192 word random access core memory, hardware multiply/divide, two clocks, and 16 levels of automatic priority interrupt. The 18 bit word allows the user to directly address 8192 core locations while executing a memory reference instruction. The computer has a basic read/restore cycle time of $1.75 \mu \mathrm{sec}$, and an average divide time of $11 \mathrm{usec}$. 
It is difficult to precisely size the digital computer in terms of analog computing blocks. However, the digital computer replaces the following analog elements on our largest hybrid simulation:

180 amplifiers

17 integrators

35 variable product multipliers

120 POTS

30 function generators

8 variable time delays (which could not be done by all analog means)

\section{CENTRAL PROCESSOR MODIFICATIONS}

The central processor has been modified to perform added arithmetic functions. (29) Changes include the addition of the instructions, Compare and Three-way Branch (CAS), One's Complement Subtract (SUB), and Increment the Accumulator (IAC). These three instructions are referred to as the modified instruction set. Their use is controlled by two IOT instructions: MIST and MICL (modified instruction "set," and modified instructions "clear").

Two clocks have been installed in the central processor. The first is crystal controlled with a frequency of $5 \mathrm{kHz}$, and the second operates from line frequency of $60 \mathrm{~Hz}$. Both clocks operate through the Automatic Priority Interrupt as interval times.

\section{PERIPHERAL DEVICES}

The PDP-7 has associated with it peripheral devices that allow input and output of user programs and data. A simplified block diagram of the system is shown in Figure 6.

A mode1 KSR 35 Teletypewriter is interfaced to the computer and operates in simplex or duplex mode and off-1ine.* Standard PDP-7 programs using the KSR 35 have been modified to use automatic vertical and horizontal tabulation and form feed.

A 300 character per second paper tape reader and 63 line per second paper tape punch provide high speed paper tape input/output with the PDP-7. These units use fan-fold paper tape having eight channels of data processed in either alphanumeric or binary modes.

A dual Dectape, Type 555, is coupled to the digital computer. Each reel of tape contains three million bits of information, and data is transferred at the rate of 90,000 bits per second.

* A 300 zine per minute zine printer wizl be incorporated into the system by January of 1969 . 


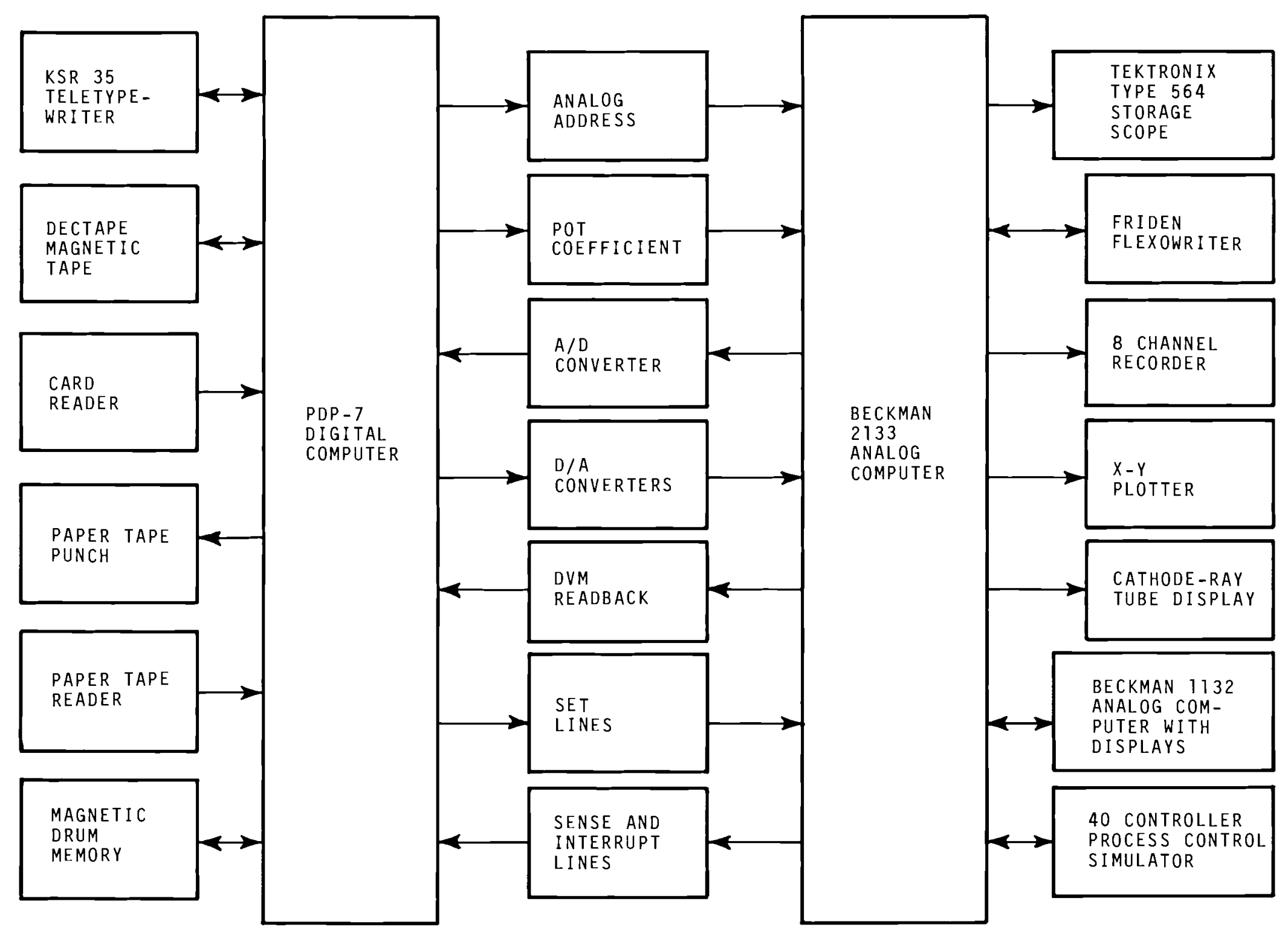


A 25,000 word drum memory (having an average access time of $20 \mathrm{msec}$, and a transfer rate of 50,000 words per second) is used for bulk storage of user programs. The drum transfers blocks of data to and from core memory via the Data Break facility of the PDP-7.

Source program card decks and data cards may be input to the PDP-7 through an IBM 713 card reader. It reads cards under program control at the rate of 150 cards per minute. Cards are used when off-1ine program preparation or online program modification is desired.

HYBRID CONTROL INTERFACE

The data interface includes a 24-channel high-speed multiplexer, an analog-to-digital converter, and 24 digital-to-analog converters. In addition, the interface allows the digital computer to perform the following control functions: (1) set analog computer modes, (2) sense analog computer mode status, (3) address variables in the analog computer, (4) read the value of a variable into core memory using the digital voltmeter, and (5) set analog computer potentiometers.

\section{ACKNOWLEDGMENTS}

The work reported in this document is the result of a team effort by the Process Simulation and Analysis Section. The efforts of Mr. L. H. Gerhardstein in conceiving, designing, and developing the major portion of the software system is of paramount importance. Second to Mr. Gerhardstein's efforts, are those of Mr. W. F. Lenzke who developed the POT Editor, List Deck, Punch Deck, Card Read, and assisted in the development of several other systems programs. Mr. Lenzke also provided support to Mr. M. D. Erickson and Mr. K. M. Busness in designing and fabricating the computer hardware for the card reader, magnetic drum, and hybrid control interface.

Mr. C. R. Cole developed four SIMPL-1 subroutines, solved the illustrative example problems, and contributed valuable ideas on organizing the appendix. Mr. H. F. Foote developed one of the SIMPL-1 subroutines and Mr. P. J. Dionne provided the MIMIC computer running times for comparison with SIMPL-1. Mr. G. R. Taylor developed the analog computer maintenance routines and the $\mathrm{AD} / 4$ analog simulation of the Pilot Ejection Study for comparative purposes. Finally, Mr. G. A. Worth supplied the discussion on assembly language methods for multiplication and division. 


\section{LITERATURE}

\section{BENCH MARK PROBLEMS}

1. Brennan, R. "Digital Simulation," presented at National Simulation Councils Meeting, AtZantic City, New Jersey. May 3, 1968.

2. Giese, C. "Determination of Best Kinetic Coefficients of a Dynamic Chemical Process by on Line Digital Simulation," Simulation, vol. 8, no. 3, pp. 141-145. March 1967.

3. Nesbit, R. A., and R. D. Engel. "An Example Program for the Determination of Chemical Rate Coefficients from Experimental Data," Simulation, vol. 8, no. 3, pp. 133-137. March 1967.

4. McLeod, J. "PHYSBE--A Physiological Simulation Bench Mark Experiment," Simulation, vol. 6, no. 7, pp. 324-329. December 1966.

\section{AMPLITUDE SCALING AIDS}

5. Harnett, R. T., F. S. Sansom, and L. M. Warshawsky. "MIDAS--An AnaZog Approach to Digital Computation," Simulation, vol. 3, no. 3, pp. $16-43$. september 1964.

6. Borgin, G. H. "APACHE--Some Encouraging Experiences," Simulation, vol. 6, no. 1, p. 16. January 1966.

\section{SOME DIGITAL ANALOG SIMULATORS}

7. Levine, L. "The DES-1, A New Digital Computer for Solving Differential Equations," Simulation, vol. 4, no. 4, p. 264. Apriz 1965.

8. Brennan, R. D. "PACTOLUS--A Simulator Language Which Makes a Digital Computer Feel Like an Analog Computer (SortaKinda)," Simulation, vol. 3, no. 2, pp. 13-19. August 1964.

9. Sansom, F.J., and H. E. Petersen. Mimic Programming Manual, SEG-TR-6731. Research and Technology Division Systems Engineering Group, WrightPatterson $A F B$, Ohio. July 1967.

10. Strauss, J. C. "The SCi Continuous System Simulation Language (CSSL)," Simulation, vol. 9, no. 6, pp. 281-303. December 1967.

11. MIMAC-A Digital System for Continuous Simulation, MeDonnell Automation

\section{RECOMMENDED ANALOG COMPUTER REFERENCES}

12. Strong, J. D. and G. Hannauer. "A Practical Approach to Analog Computers," Instruments \& Control Systems, vol. 35, pp. 60-71. August 1962.

13. Parisot, P. E. "Analog Computers," Chemical Engineering, p. 137. September 1959 .

14. Johnson, C. L. Analog Computer Techniques, Second Edition, McGraw-Hizl, New York, 1963

15. Truitt, T. D., and A. E. Rogers. Basics of Analog Computers, Rider, Inc., New York. 1960.

16. Rogers, A. E. and T. W. Connolzy. Analog Computation in Engineering Design, McGraw-Hizl. 1960. 


\section{RECOMMENDED DIGITAL COMPUTER REFERENCES}

17. Benrey, R. Understanding Digital Computers, Rider, Inc., New York. 1967.

18. Murphy, J. A. Basics of Digital Computers, vol. 1, 2, 3, Rider, Inc., New York. 1958 .

19. MeCracken, D. D. Digital Computer Programming, John Wiley \& Sons, Inc., New York. 1963.

20. Stein, M. L., and W. D. Munro. Computer Programming, Academic Press, New York. 1964.

21. Knight, K. E. "Changes in Computer Performance," Datamation, vol. 12, no. 9. September 1966.

\section{LITERATURE APPLICABLE TO SIMPL-1}

22. Instructions--2100 Series operator's Manual, Beckman Instruments, Inc., Berkeley, California. August 1964 .

23. PDP-7--Users Handbook, F-75, Digital Equipment Corporation, Maynard, Massachusetts. 1965 .

24. "PDP-7--Symbolic Tape Editor," Digital-7-1-S, Digital Equipment Corporation, Maynard, Massachusetts. 1965.

25. "PDP-7--DDT Debugging System Programming Manual," Digital-7-4-S, Digital Equipment Corporation, Maynard, Massachusetts. $19 \overline{65}$.

26. "PDP-7--FORTRAN II," Digital-7-2-S, Digital Equipment Corporation, Maynard, Massachusetts. 1965 .

27. "PDP-7--DECSYS-7," Digital-7-5-S, Digital Equipment Corporation, Maynard, Massachusetts. $196 \overline{5}$.

28. Erickson, M. D. The Pacific Northwest Laboratory Hybrid Computer System, BNWL-538. Pacific Northwest Laboratory, Richland, Washington. January 1968.

29. Draper, W. D. A Modified Instruction Set for the FFTF Instrumentation Prototype, BNWL-451. Pacific Northwest Laboratory, Richland, Washington. August 196 ?.

30. Busness, K. M. Hybrid Computer-Card Reader Interface, BNWL-CC-1671. Pacific Northwest Laboratory, Richland, Washington. June 3, 1968.

31. Busness, K. M. A Magnetic Drum Interface for the PNL Hybrid Computer Facility, BNWL-CT-1651. Pacific Northwest Laboratory, Richland, Washington. May 30, 1968 .

32. Digital Logic Handbook, pp. 12-15, Digital Equipment Corporation, Maynard, Massachusetts. 1967 .

33. Gerhardstein, L. H. Unpublished Data. Pacific Northwest Laboratory, Richland, Washington. To be published 1968. (Froposed title: SIMPL-1 Systems Technical Manual)

34. Tayzor, G. R. Unpublished Data. Pacific Northwest Laboratory, Richland, Washington. (Proposed title: Analog Diagnostic Program)

35. Lenzke, W. F. Unpublished Data. Pacific Northwest Laboratory, Richland, Washington. (Proposed title: POT Editor) 


\section{APPENDICES}




\section{NAMES}

The Table below shows that names (or tags), reference storage locations where constants, variables, instructions or other names are stored.

\begin{tabular}{|c|c|c|c|}
\hline \multirow[b]{2}{*}{ TYPE OF NAME } & \multicolumn{3}{|c|}{ PROGRAM LISTING } \\
\hline & TAG & STORED VALUE & \begin{tabular}{|c|} 
COMMENTS \\
\end{tabular} \\
\hline Constant* & PIE, & 3.14 & $\begin{array}{l}\text { The constant } \pi \text { is } \\
/ \text { stored (in octal form) } \\
/ \text { in storage location PIE }\end{array}$ \\
\hline Variable & $\mathrm{T}$, & $\emptyset$ & $\begin{array}{l}\text { /A variable such as } \\
\text { / temperature is stored } \\
\text { /in location called } \mathrm{T}\end{array}$ \\
\hline Instruction & & JMS JOE & $\begin{array}{l}\text { /The instruction JMS JOE is } \\
\text { / stored in location WHERE }\end{array}$ \\
\hline Name & $X Y$, & TBL1. & $\begin{array}{l}\text { /Location } \mathrm{XY} \text { is where the } \\
\text { /name TBLl is stored }\end{array}$ \\
\hline
\end{tabular}

*Names can also stand for constants, For example:

$$
\text { PIE }=3.14 \text {. }
$$

Whenever PIE is used in the program the assembler replaces it with the octal equivalent of 3.14

** Storage locations for named variables containing a \# or $>$ are automatically assigned by the assembler.

Symbolic names (or address tags) may use up to six letters and digits. Characters following the sixth symbol of a name are ignored (eg. the symbolic name CONVERT is interpreted as CONVER). The first character of a name must be:

$$
\begin{aligned}
& \text { 1. Letter (A through Z) } \\
& \text { 2. Period (.) } \\
& \text { 3. Dollar slgn (\$) }
\end{aligned}
$$

The remaining characters can be a letter, period, dollar sign or a number ( thru 9). The dollar sign and perlod also have special assembler functions. The period is used as a voltage indicator when preceeded by a digit or as a current address indicator when preceeded by a space and followed by a space, $t \in b$, carriage return, + , or - sign. The dollar sign is normally reserved for user and library subroutine communication. (Pseudo instructions can be used for address tags or variable names.)

Examples:

$$
\begin{aligned}
& \text { \$GL1 } \\
& \text { TEMP } \\
& \text { POWER1 } \\
& \text { SPHEAT }
\end{aligned}
$$$$
.60
$$

NUMBERS

A number is any grouping of six (6) or less digits excluding voltage (.) or decimal (") indicators. For example:

$\begin{array}{cl}100^{\prime} & \text { (decimal integer) } \\ 1599.99 & \text { (voltage) } \\ 354617 & \text { (octal integer) } \\ 0.52 & \text { (voltage) } \\ .52 & \text { (a name not a number because a digit } \\ & \text { to the left of decimal point was } \\ & \text { omitted) }\end{array}$

CONTROL

The arithmetic and program control characters are summarized below:

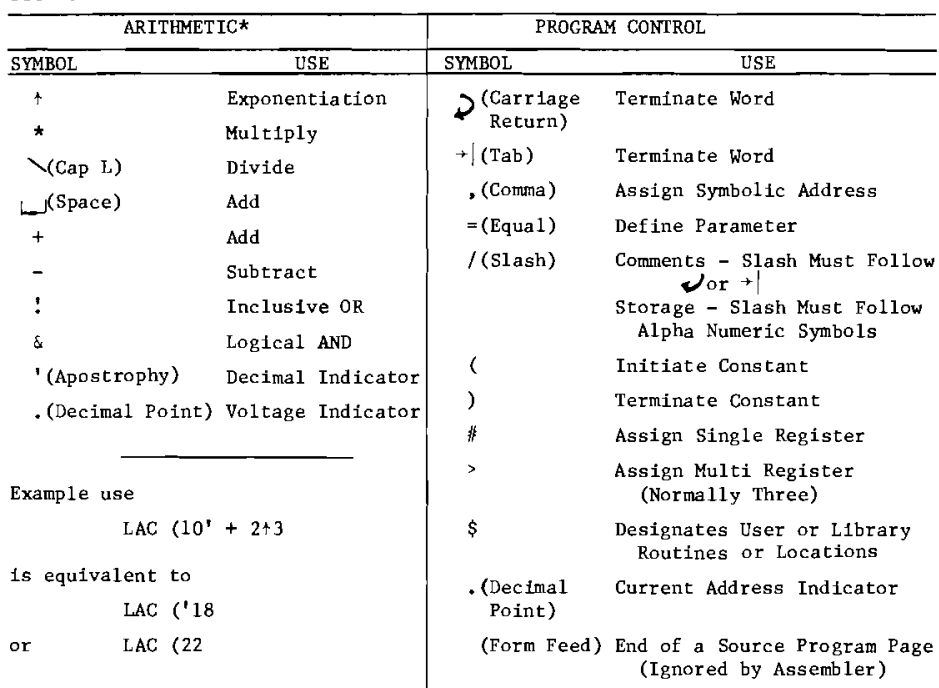

*or a detailed explanation of most of the arithmetic and control functions refer to the PDP-7 symbolic assembler programuing manual. Differences between the SIMPLI instructions and DEC's instructions reflect changes to the standard sof tware system.

The example shown as a footnote to this arithmetic section shows how literal constants may be represented. Care must be exercised in using these symbols to insure producing the right numbers. For example:

$$
10.0 \uparrow 3 \neq 1000.0 \text { volts }
$$

here 1s why

$$
\text { The decimal equivalent of } \begin{aligned}
10.0 \text { volts } & =\left(\frac{2^{13}}{100}\right) 10^{\prime} \\
& =C^{\star} 10^{\prime}
\end{aligned}
$$

Therefore

$$
10.0 \uparrow 3=\left(C^{\star} 10^{\prime}\right)^{3}=\left(\frac{2^{13}}{100}\right)^{3}\left(10^{\prime}\right)^{3}=\left(\frac{2^{13}}{100}\right)^{2}(1000 .)
$$

However, the result shown in the accumulator would be garbage because of accumulator overflow. The largest number permitted is 1599.99. If 1000.0 (volts were desired, the following notation could be used.

$$
\begin{aligned}
1.0 * 10^{\prime}+3 & =\left(C^{\star} 1\right) \star 10^{\prime} \uparrow 3 \\
& =C \times 1000=1000 .
\end{aligned}
$$

If the voltage 40.0 were desired the following sequence could be used:

$$
1000 \backslash \backslash 25=(C)(1000) \backslash 25=(C)(40)=40 .
$$

If the voltage 43 were desired the following sequence could be used: $1000 . \backslash^{\prime} 25+3 .=43$.

$$
\text { because } \quad \frac{(\mathrm{C})\left(1000^{\prime}\right)}{25^{\prime}}+(\mathrm{C})\left(3^{\prime}\right)=\mathrm{C}\left(40^{\prime}\right)+\mathrm{C}\left(3^{\prime}\right)=43 \text {. }
$$

In order to var1fy if an arithmetic assembler operation will produce the desired number a numerfcal unit analysis similar to that given above should be performed.

Assembler arithmetic operations cannot be used for multiplying problem varfables. They can be used for multiplying constants. (Variable multiplication is accomplished with SIMPLl library routines like ERPY $2 \$$. ) 


\section{APPENDIX B}

\section{LIST OF SIMPL-1 FUNCTIONS}

\begin{tabular}{lll} 
FUNCTION & $\begin{array}{l}\text { SYMOLIC } \\
\text { NAME }\end{array}$ & CALLING \\
& SEQUENCE & REMARKS \\
\hline
\end{tabular}

1. ARITHMETIC FUNCTIONS

\begin{tabular}{|c|c|c|c|}
\hline ADDITION & $\mathrm{ADD}$ & $\begin{array}{l}\text { LAC X } \\
\text { ADD } Y\end{array}$ & $\begin{array}{l}\text { 1. } \mathrm{TIME}=3.5 \mu \mathrm{sec} \\
\text { 2. } \mathrm{AC}=\mathrm{X}+\mathrm{Y} \\
\text { 3. See Hybrid Instruction Set }\end{array}$ \\
\hline SUBTRACTION & SUB & $\begin{array}{ll}\text { LAC } & X \\
\text { SUB } & Y\end{array}$ & $\begin{array}{l}\text { 1. } \text { TIME }=3.5 \mu \mathrm{sec} \\
\text { 2. } A C=X-Y \\
\text { 3. See Hybrid Instruction Set } \\
\text { 4. MIST must preceed sub-instruction }\end{array}$ \\
\hline NEGATION & CMA & CMA & 1. See Hybrid Instruction Set \\
\hline MULTIPLY & HMPY1\$ & $\begin{array}{l}\text { LAC X } \\
\text { JMS HMPY1\$ } \\
\text { Y } \\
\cdots\end{array}$ & $\begin{array}{l}\text { 1. } \operatorname{TIME}=33-38 \mu \mathrm{sec} \\
\text { 2. } \mathrm{AC}=\frac{\mathrm{X} * \mathrm{Y}}{100} \\
\text { 3. } \mathrm{Y}<100\end{array}$ \\
\hline MULTIPLY & HMPY $2 \$$ & $\begin{array}{l}\text { LAC X } \\
\text { JMS HMPY } 2 \$ \\
\text { LAC Y } \\
\cdots \cdots\end{array}$ & $\begin{array}{l}\text { 1. } \mathrm{TIME}=35-40 \mu \mathrm{sec} \\
\text { 2. } \mathrm{AC}=\frac{\mathrm{X} * \mathrm{Y}}{100}\end{array}$ \\
\hline MULTIPLY & HMPY3\$ & $\begin{array}{l}\text { LAC X } \\
\text { JMS HMPY } 3 \\
\text { LAC Y } \\
\text { LAC Z } \\
\ldots \ldots\end{array}$ & $\begin{array}{l}\text { 1. } \mathrm{TIME}=59-67 \mu \mathrm{sec} \\
\text { 2. } \mathrm{AC}=\frac{X^{\star} \mathrm{Y}}{2}\end{array}$ \\
\hline DIVIDE & HDIV\$ & $\begin{array}{l}\text { LAC X } \\
\text { JMS HDIV } \$ \\
\text { LAC Y } \\
\cdots \cdots\end{array}$ & $\begin{array}{l}\text { 1. } \text { TIME }=47 \mu \mathrm{sec} \\
\text { 2. } \mathrm{AC}=100 \frac{\mathrm{X}}{\mathrm{Y}}\end{array}$ \\
\hline MULTIPLY BY 10 & $\mathrm{X} \emptyset \emptyset \$$ & $\begin{array}{l}\text { LAC } \mathrm{X} \\
\text { JMS XIØS } \\
\cdots\end{array}$ & $\begin{array}{l}\text { 1. } \mathrm{TIME}=19-23 \mu \mathrm{sec} \\
\text { 2. } \mathrm{AC}=10 * \mathrm{X}\end{array}$ \\
\hline MULTIPLY BY 5 & $x 5 \$$ & $\begin{array}{l}\text { LAC } \mathrm{X} \\
\text { JMS } \times 5 \$ \\
\cdots \cdots\end{array}$ & $\begin{array}{l}\text { 1. } \mathrm{TIME}=20-23 \mu \mathrm{sec} \\
\text { 2. } \mathrm{AC}=5 * \mathrm{X}\end{array}$ \\
\hline MULTIPLY BY 2 & $\mathrm{X} 2 \$$ & $\begin{array}{l}\text { LAC } \mathrm{X} \\
\text { JMS } \mathrm{X} 2 \$ \\
\cdots\end{array}$ & $\begin{array}{l}\text { 1. } \mathrm{TLME}=20 \mu \mathrm{sec} \\
\text { 2. } \mathrm{AC}=2 * \mathrm{X}\end{array}$ \\
\hline MULTIPLY BY 0.5 & $\mathrm{X} .5 \$$ & $\begin{array}{l}\text { LAC } X \\
\text { JMS X.5\$ } \\
\cdots\end{array}$ & $\begin{array}{l}\text { 1. } \mathrm{TIME}=12 \mu \mathrm{sec} \\
\text { 2. } \mathrm{AC}=0.5 * \mathrm{X}\end{array}$ \\
\hline MULTIPLY BY 0.2 & $\mathrm{x} .2 \$$ & $\begin{array}{l}\text { LAC X } \\
\text { JMS X.5\$ } \\
\ldots \ldots\end{array}$ & $\begin{array}{l}\text { 1. } \text { TIME }=9-13 \mu \mathrm{sec} \\
\text { 2. } \mathrm{AC}=0.5 * \mathrm{X}\end{array}$ \\
\hline MULTIPLY BY 0.1 & $\mathrm{x.1 \$}$ & $\begin{array}{l}\text { LAC } \mathrm{X} \\
\text { JMS } \mathrm{X} \cdot 1 \$ \\
\ldots \ldots\end{array}$ & $\begin{array}{l}\text { 1. } \mathrm{TIME}=9-13 \mu \mathrm{sec} \\
\text { 2. } \mathrm{AC}=0.1 * \mathrm{X}\end{array}$ \\
\hline
\end{tabular}




\section{LIST OF SIMPL-1 FUNCTIONS (contd)}

\begin{tabular}{|c|c|c|c|}
\hline FUNCTION & $\begin{array}{l}\text { SYMBOLIC } \\
\text { NAME } \\
\end{array}$ & $\begin{array}{l}\text { CALLING } \\
\text { SEQUENCE } \\
\end{array}$ & REMARKS \\
\hline \multicolumn{4}{|l|}{ 2. TRANSCENDENTALS } \\
\hline SQUARE & HSQS & $\begin{array}{l}\text { LAC } \mathrm{X} \\
\text { JMS HSQS } \\
\cdots \cdots\end{array}$ & 1. $\begin{aligned} & \text { TIME }=28 \mu \mathrm{sec} \\
& \text { 2. } \begin{aligned} \text { AC } & =\frac{x^{2}}{100}, x<399.99 \\
& =1599.99, x \geq 399.99\end{aligned}\end{aligned}$ \\
\hline CUBE & HCUBES & $\begin{array}{l}\text { LAC X } \\
\text { JMS HCUBES } \\
\cdots \cdots\end{array}$ & $\begin{array}{l}\text { 1. } \text { TIME }=45 \mu \mathrm{sec} \\
\text { 2. } \begin{aligned} \mathrm{AC} & =\frac{\mathrm{x}^{3}}{1000}, \mathrm{X}<251.97 \\
& =1599.99, \mathrm{X} \geq 251.97\end{aligned}\end{array}$ \\
\hline SQUARE ROOT & HSQRT\$ & $\begin{array}{l}\text { LAC X } \\
\text { JMS HSQRT } \\
\ldots \ldots\end{array}$ & $\begin{array}{l}\text { 1. } \text { TIME }=90 \mu \mathrm{sec} \\
\text { 2. } \mathrm{AC}=10 \sqrt{\mathrm{X}}, \mathrm{X}>0\end{array}$ \\
\hline COMMON LOG & HLOG\$ & $\begin{array}{l}\text { LAC X } \\
\text { JMS HLOG } \$ \\
\ldots \ldots\end{array}$ & $\begin{array}{l}\text { 1. } \mathrm{TIME}=100 \mu \mathrm{sec} \\
\text { 2. } \mathrm{AC}=100 \operatorname{LOG}_{\mathrm{e}} \mathrm{X}, \mathrm{X} \geq 0\end{array}$ \\
\hline $\begin{array}{l}\text { EXPONENT } \\
\text { (BASE 10) }\end{array}$ & HEXP\$ & $\begin{array}{l}\text { LAC X } \\
\text { JMS HEXPS } \\
\ldots \ldots\end{array}$ & 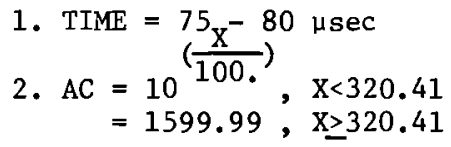 \\
\hline COSINE & $\mathrm{HCOS} \$$ & $\begin{array}{l}\text { LAC X } \\
\text { JMS HCOS } \$ \\
\ldots \ldots\end{array}$ & $\begin{array}{l}\text { 1. } \text { TIME }=70-90 \mu \mathrm{sec} \\
\text { 2. } \mathrm{AC}=100 \cos \left(\frac{\pi}{2} * \frac{\mathrm{X}}{100}\right) \\
\left(100 .=90^{\circ}\right)\end{array}$ \\
\hline SINE & HSIN\$ & $\begin{array}{l}\text { LAC X } \\
\text { JMS HSINS } \\
\ldots \ldots\end{array}$ & $\begin{array}{l}\text { 1. } \text { TIME }=77-97 \mu \mathrm{sec} \\
\text { 2. } \mathrm{AC}=100 \operatorname{SIN}\left(\frac{\pi}{2} * \frac{\mathrm{X}}{100}\right)\end{array}$ \\
\hline QUANTIZER & QUANS & $\begin{array}{l}\text { LAC X } \\
\text { JMS QUAN\$ } \\
\text { A } \\
\ldots\end{array}$ & $\begin{array}{l}\text { 1. } \operatorname{TIME}=45 \mu \mathrm{sec} \\
\text { 2. } \mathrm{AC}=\mathrm{A} * \cap\left\{\frac{\mathrm{X}}{\mathrm{A}}\right\}\end{array}$ \\
\hline LIMITER (+ and -) & LIMIT\$ & $\begin{array}{l}\text { LAC } \mathrm{X} \\
\text { JMS LIMIT\$ } \\
\text { A } \\
\text { B }\end{array}$ & 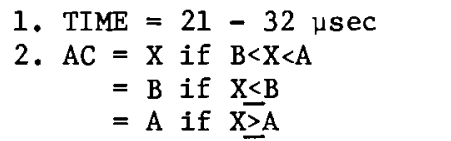 \\
\hline
\end{tabular}

3. LOGICAL FUNCTIONS (See PDP-7 Instruction Set)

\begin{tabular}{|c|c|c|c|}
\hline AND & AND & $\begin{array}{l}\text { LAC X } \\
\text { AND } Y\end{array}$ & $\begin{array}{l}\text { 1. } \mathrm{TIME}=3.5 \mu \mathrm{sec} \\
\text { 2. } \mathrm{AC}=\mathrm{A} \Omega \mathrm{B}\end{array}$ \\
\hline EXCLUSIVE OR & XOR & $\begin{array}{l}\text { LAC X } \\
\text { XOR Y }\end{array}$ & $\begin{array}{l}\text { 1. } \text { TIME }=3.5 \mu \mathrm{sec} \\
\text { 2. } \mathrm{AC}+\left(\mathrm{A} \Omega \mathrm{B}^{\prime}\right) \mho\left(\mathrm{A}^{\prime} \Omega \mathrm{B}\right)\end{array}$ \\
\hline COMPARE & CAS & $\begin{array}{l}\text { LAC X } \\
\text { CAS Y }\end{array}$ & $\begin{array}{l}\text { 1. } \text { TIME }=3.5 \text { usec } \\
\text { 2. MIST Instruction Must Preceed CAS } \\
\text { 3. if } A C>Y \text { no skip } \\
\begin{aligned} A C & =Y \text { skip } 1 \text { instruction } \\
A C & <Y \text { skip } 2 \text { instructions }\end{aligned}\end{array}$ \\
\hline
\end{tabular}




\section{LIST OF SIMPL-1 FUNCTIONS (contd)}

$\begin{array}{lll}\text { FUNCTION } & \begin{array}{c}\text { SYMBOLIC } \\ \text { NAME }\end{array} & \text { CALLING } \\ \text { SEOUENCE }\end{array}$

REMARKS

4. FUNCTION GENERATORS

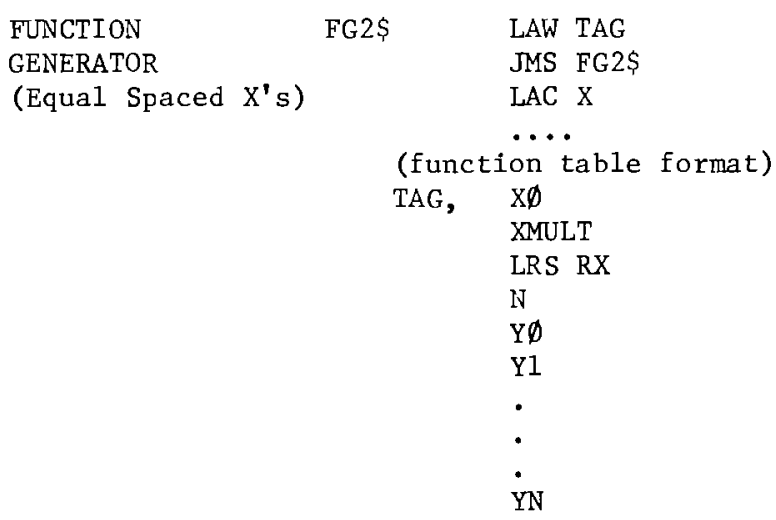

1. $\mathrm{TIME}=93-104 \mu \mathrm{sec}$

2. $A C=F(X)$

3. Equal spaced points

4. Linear interpolation between points

(function table format) 5. $\mathrm{L}=1$ if $\mathrm{X}$ is outside of function table

6. $X \emptyset=$ First $X$ coordinate

$\mathrm{N}=$ No. of segments in function

$\mathrm{Y} \emptyset=$ First $\mathrm{Y}$ coordinate

7. Obtain XMULT(a voltage) and "RX" from table below

$$
\text { XMULT }=A \frac{(\text { Table Value })}{\Delta X}
$$

Table for calculating values for "MULTIPLIER" and 'SHIFT COUNT" for FG2\$ \& FG3\$

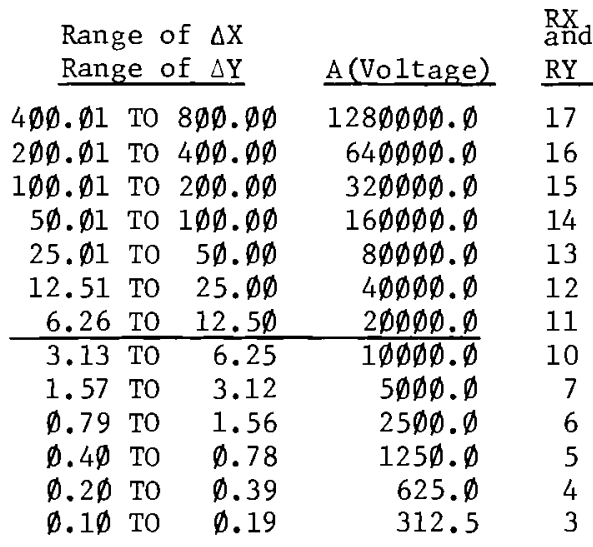

Example for FG2\$:

If $\Delta \mathrm{X}$ were chose to be 10 volts, the value of "RX" would be 11 and

$$
\text { XMULT }=\frac{20000}{10} \cdot=2000 \text {. }
$$

Example for FG3\$:

If $\Delta \mathrm{X}$ were chose to be 30 volts and $\Delta \mathrm{Y} 1$ volt then from the table

$$
\begin{aligned}
\mathrm{RX} & =13 \\
\mathrm{XMULT} & =\frac{40000}{30} \cdot=1333.33 \\
\mathrm{RY} & =6 \\
\text { YMULT } & =\frac{2500}{1 .}=2500 .
\end{aligned}
$$


LIST OF SIMPL-1 FUNCTIONS (contd)

\section{FUNCTION \\ FUNCTION GENERATOR \\ OF TWO}

VARIABLES
CALLING

SEOUENCE

\begin{tabular}{cl}
$\begin{array}{c}\text { SYMBOLIC } \\
\text { NAME }\end{array}$ & CALLING \\
\cline { 1 - 1 } FG3\$ & SEOUENCE \\
& LAW TABLE \\
& JMS FG3\$ \\
& LAC X \\
& LAC Y \\
& $\ldots$
\end{tabular}

(function table format) TABLE, $\quad X \emptyset$

XMULT

LRS RX

$\mathrm{N}$

$\mathrm{Y} \emptyset$

YMULT

LRS RY

$\mathrm{M}$

$Z \emptyset \emptyset$

$\mathrm{Z1 \emptyset}$

-

Zø1

.

$\cdot$

ZN1

•

$\cdot$

$\cdot$

Zंग

Z1M

-

$\cdot$

ZNM

FUNCTION

GENERATOR

(Arbitrary Spaced $X^{\prime} s$ )
FG1\$

G1\$

LAC $\mathrm{X}$ JMS FG1\$

TAG

$$
\text { .... }
$$

(function table format) TAG,
$\mathrm{N}$

$\mathrm{X} 1$

$\mathrm{X} 2$

$\dot{\bullet}$

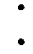

$\mathrm{XN}$

$\mathrm{Y} 1$

Y2

•

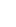

YN
REMARKS

1. TIME $=272-3 \emptyset 4 \mu \mathrm{sec}$

2. $A C=F(X, Y)$

3. $L=1$ if $X$ or $Y$ outside of function table

4. First order interpolation between equally spaced points ( $\Delta \mathrm{X}$ and $\Delta \mathrm{Y}$ can be different)

5. See table for determining

$\mathrm{RX}$

$$
\text { XMULT }=\frac{A_{x}}{X}
$$

RY

$$
\text { YMULT }=\frac{A}{Y}
$$

6. $N=$ Number of $X$ segments

$=1$-number of $X$ points

$M=$ Number of $Y$ segments

$=1$-number of $\mathrm{Y}$ points

7. TABLE FORMAT

\begin{tabular}{c|c|c|c|c} 
& $\mathrm{Y}_{0}$ & $\mathrm{Y}_{1}$ & $\cdot \cdot \cdot \cdot$ & $\mathrm{Y}_{\mathrm{M}}$ \\
\hline $\mathrm{X}_{0}$ & $\mathrm{z}_{00}$ & $\mathrm{z}_{01}$ & $\cdot \cdot \cdot \cdot$ & $\mathrm{z}_{\mathrm{OM}}$ \\
\hline $\mathrm{X}_{1}$ & $\mathrm{z}_{10}$ & $\mathrm{z}_{11}$ & $\cdot \cdot \cdot \cdot$ & $\mathrm{Z}_{1 \mathrm{M}}$ \\
\hline$\cdot$ & $\cdot$ & $\cdot$ & $\cdot$ & \\
$\cdot$ & $\cdot$ & $\cdot$ & $\cdot$ & \\
$\cdot$ & $\cdot$ & $\cdot$ & $\cdot$ & \\
\hline $\mathrm{X}_{\mathrm{N}}$ & $\mathrm{Z}_{\mathrm{N} 0}$ & $\mathrm{Z}_{\mathrm{N} 1}$ & & $\mathrm{Z}_{\mathrm{NM}}$
\end{tabular}




\section{LIST OF SIMPL-1 FUNCTIONS (contd)}

\begin{tabular}{|c|c|c|c|}
\hline FUNCTION & $\begin{array}{l}\text { SYMBOLIC } \\
\text { NAME } \\
\end{array}$ & $\begin{array}{l}\text { CALLING } \\
\text { SEQUENCE } \\
\end{array}$ & REMARKS \\
\hline $\begin{array}{l}\text { FUNCTION } \\
\text { GENERATOR }\end{array}$ & $\begin{array}{l}\text { (fur } \\
\text { TAG }\end{array}$ & 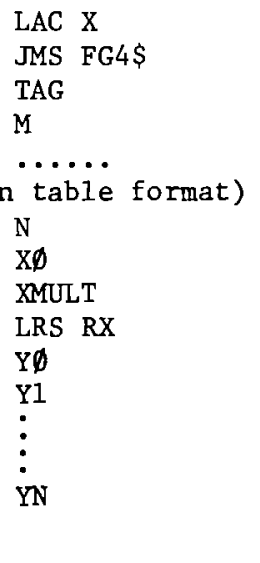 & $\begin{array}{l}\text { 1. TIME }=250+100 * M \\
\text { 2. } A C=F(X) \\
\text { 3. Equal spaced points } \\
\text { 4. Value of } M \text { determines order of interpolation. } \\
M \text { may have values from } 1 \text { to } 5 \\
\text { 5. } X \emptyset=\text { first } X \text { coordinate } \\
\mathrm{N}=\text { No. of segments in function } \\
Y \emptyset=\text { First } Y \text { coordinate } \\
\text { 6. Obtain XMULT and RX from same table used } \\
\text { for FG2\$ } \\
\qquad X M U L T=\frac{A(\text { from table) }}{\Delta X} \\
\text { 7. Can be used for cross plotting } \\
\text { (For straight line interpolation use } \\
\text { FG1\$ or FG2\$). }\end{array}$ \\
\hline
\end{tabular}

\section{TIME DEPENDENT FUNCTIONS}

\section{CALCULATE CNP\$ \\ NUMBER OF \\ CLOCK PULSES}

ASYNCHRONOUS OPERATION

(For problems that do not use a clock service subroutine)
41) ISZ \$CL1
501 ISZ \$CL2
:
JMS CNP\$
$\emptyset$
...
1. TIME $=47-67 \mu \mathrm{sec}$
2. Clock and API must be turned on as required in the program
3. Can be used with RINT\$
4. $A C=N_{p}$ (number of clock pulses)
5. The location following JMS CNP\$(i.e. 0 ) holds the last clock value.

SYNCHRONOUS OPERATION

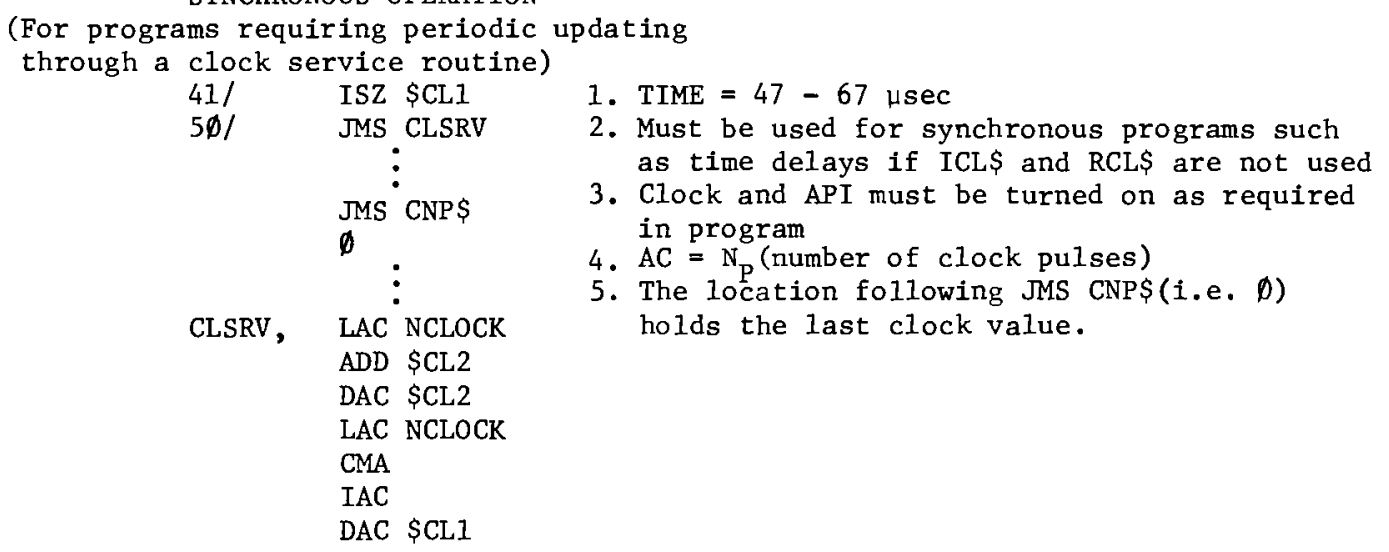




\section{LIST OF SIMPL -1 FUNCTIONS (contd)}

FUNCTION

INITIALIZE

REAL-TIME

CLOCK
RELEASE CLOCK API CHANNEL

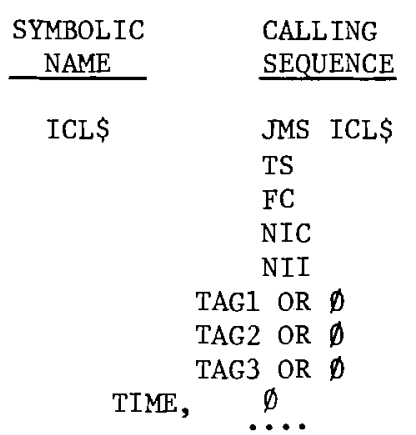

\section{REMARKS}

1. TTME $=135+$ TIME IN TAG1, etc.

2. To be used in program initialization to

a. Set the clock frequency location \$FC

b. Set the time scale location \$TS

c. Initialize the clock API hardware

d. Compute clock counters for compute and IC modes

e. Establish user program locations for performing synchronized time dependent operations (time-delay, etc.)

f. Establish a location (TIME) in the users program that will contain the time (voltage scaling) since compute mode was entered. (The digital computer mode is entered by storing the number 4 in location MODES or by using JMS AMODE\$ to check if analog computer is in compute.) The time scale (TS) is taken into account in computing this value.

g. Save all hardware registers (AC, link, etc.) at interrupt time

3. The number of computing frames is given by:

Time (voltage scaling) Loop time/(computing fraction)

where the computing fraction is:

$\frac{\frac{10^{6}}{(\mathrm{NIC})(\mathrm{TS})}-\left(135+\mathrm{TIME} \text { in TAG1, tc. }-\frac{(\mathrm{FC})(3.5)}{(\mathrm{NIC})(\mathrm{TS})}\right.}{\frac{10^{6}}{(\mathrm{NIC})(\mathrm{TS})}}$

4. The digital computer should calculate at leat 10-20 values of each variable per cycle of its highest frequency.

5. NOMENCLATURE

$\mathrm{TS}=$ Time scale (any number $>1$ )

$\mathrm{FC}=$ Clock frequency $\left(60^{\circ}\right.$ or $\left.5 \emptyset \emptyset \emptyset^{\prime}\right)$

NIC $=$ Number of iterations $/ \mathrm{sec}$ in compute mode

NII = Number of iterations/sec in IC mode

TAG1 = TAG in user's program requiring interrupt service during compute mode ( $\emptyset$ for no action)

TAG2 = TAG in user's program requiring interrupt service during IC mode ( $\emptyset$ for no action)

TAG3 = TAG in user's program requiring interrupt service during HOLD mode ( $\emptyset$ for no action)

TIME = LOCATION CONTAINING TIME (in sec of voltage scaling) since entering compute modes

1. TIME $=$

2. When using the subroutine ICL\$, the programmer can specify a location where control should be transferred for time dependent operations. (See TAG1, TAG2, or TAG3, in the ICL\$ 1isting). The last instruction of the time dependent subroutine must be a JMP RCL\$ instruction 


\section{LIST OF SIMPL-1 FUNCTIONS (contd)}

\section{FUNCTION}

TIME DELAY
SYMBOLIC NAME

TD1\$
CALLING

SEQUENCE

LAW TAG

JMS TD1Ş

$\emptyset$

$\emptyset$

LAC W
XX
$\emptyset$
$\emptyset$
$\emptyset$
DAC.-2
LAC YIN
HLT
$\ldots .$.
table format)
TAU
LAC (NI
N
WMAX

TAG+N+4/

RECTANGULAR INTEGRATION
RINTS

LAW .

JMP RINTS

LAC \$MODE

LAC NP

LAC Y $\emptyset$

LAC $\mathrm{X}$

LLSS $K$ or LRSS $K$

ADD Y

$\emptyset$

DAC Y

:

NOP

JMP . +1

\section{REMARKS}

1. TIME $=240+10.5 * \mathrm{~N}$ (I.C.)

$$
=160 \text { to } 176 \text { (Compute) }
$$

2 . The program must be synchronized by the realtime clock

3. The storage location \$MODE must contain the computer mode (see note 2 given with AMODE\$).

a. The IC mode must be entered at least once to initialize the delay.

b. In the HOLD mode the delay is frozen.

c. In compute the delay works properly.

4. NOMENCLATURE

$\mathrm{NI}=$ Number of iterations per sec (problem time)

TAU = Minimum time delay (for maximum flow)

$\mathrm{N}=$ Number of segments in the delay function

$\mathrm{W}=\mathrm{Flow}$ variable

WMAX = Maximum flow (corresponds to TAU)

YIN = Input variable (temperature)

RMAX = Maximum rate of change YIN

$\lambda=$ Approximate time constant if YIN is a decaying function

$\omega=$ Approximate frequency if YIN is a sinusodial function.

$\tau_{\mathrm{MAX}}=$ Maximum time delay

ERROR = Error in function

5. DEFINING EQUATIONS

$$
\begin{aligned}
\mathrm{N} & =\left(\mathrm{R}_{\text {MAX }}\right) \frac{\tau_{\text {MAX }}}{\text { ERROR }} \\
N_{I} & =\frac{R_{\text {MAX }}}{\text { ERROR }} * \frac{W_{\text {MIN }}}{\text { WMAX }}+2
\end{aligned}
$$

( $\mathrm{N}_{\mathrm{I}}$ must be an integer multiple of $1,2,4,5$ )

$\mathrm{R}_{\text {MAX }}=\frac{\text { YIN }_{\text {MAX }}}{\lambda}$ (first order decay)

$=$ WYIN $_{(\text {MAX }}$ (second order)

6. Program will place analog computer in IC MODE and HLT digital computer if the following inequality is violated

$$
1 \geq \frac{\mathrm{N} I * \mathrm{TAU}}{\mathrm{N}}<16
$$

7. $A C=Y I N(t-\tau)$

1. $\operatorname{TIME}=57+71 * \mathrm{~N} \mu \mathrm{sec}$

where

$$
\mathrm{N}=\text { Number of integrations nested in calling }
$$
sequence

2. The mode variable must be provided according to the standards of \$MODE

3. LLSS $\mathrm{K}$ or LLRS $\mathrm{K}$ produces an integrator gain of $(2 * *(+K))$ or $(2 * *(-K))$ respectively

4. For synchronous operation

$$
\mathrm{NP}=\text { Number of clock pulses since last }
$$
calculation (see CNP\$ or ICL\$ and RCL\$)

5. $Y$ = Initial condition

$$
\mathrm{X}=\text { Input variable }
$$

$$
\left(\frac{d Y}{d t}\right)
$$

$\mathrm{Y}=$ Output variable

6. $\mathrm{AC}=\int X \mathrm{X} t+\mathrm{Y} \emptyset$

7. Any number of integrations can be nested in 


\section{LIST OF SIMPL-1 FUNCTIONS (contd)}

\begin{tabular}{|c|c|c|c|}
\hline FUNCTION & $\begin{array}{l}\text { SYMBOLIC } \\
\text { NAME }\end{array}$ & $\begin{array}{l}\text { CALLING } \\
\text { SEQUENCE } \\
\end{array}$ & REMARKS \\
\hline \multicolumn{4}{|c|}{ 6. A/D AND D/A CONVERSION \& CONTROL } \\
\hline $\begin{array}{l}\text { ANALOG TO } \\
\text { DIGITAL } \\
\text { BLOCK } \\
\text { TRANSFER }\end{array}$ & ADC\$ & $\begin{array}{l}\text { LAC } \quad \text { N } \\
\text { JMS ADC } \$ \\
\text { M } \\
\text { DAC } \quad X \\
\text { DAC Y } \\
\text { NOP } \\
\vdots \\
\text { DAC } \\
\cdots \\
\cdots\end{array}$ & $\begin{array}{l}\text { 1. TIME }=45+19.25 * \mathrm{~A}+17.5 * \mathrm{~B} \\
\text { where } \\
\mathrm{A}=\text { Number of used channels } \\
\mathrm{B}=\text { Number of unused channels in the } \\
\text { selected block } \\
\text { overload servicing time } \\
\text { used channels }=60 \mathrm{\mu sec} \\
\text { unused channels }=35 \mathrm{\mu sec} \\
\text { 2. If any channels are overloaded and the A/D over- } \\
\text { load API channel is on the channels will be } \\
\text { automatically serviced. The value is set to } \\
\pm 99.99 \text {. Upon return to the calling program } \\
\text { AC }=\text { No. of overload channels } \\
\quad \mathrm{N}=\text { First channel of block } \\
\mathrm{M}=\text { Last channel of block } \\
\text { 4. A NOP in the calling sequence can be used to } \\
\text { skip a channel. In the calling sequence given } \\
\text { above channel N } 2 \text { is not used }\end{array}$ \\
\hline $\begin{array}{l}\text { DIGITAL TO } \\
\text { ANALOG BLOCK } \\
\text { TRANSFER }\end{array}$ & DAC $\$$ & $\begin{array}{l}\text { LAC } \quad(\mathrm{N} \\
\text { JMS DAC } \$ \\
\text { M } \\
\text { LAC X } \\
\text { LAC Y } \\
\text { SKP } \\
\vdots \\
\text { LAC }(-25 . \emptyset \emptyset \\
\text { LAC Z } \\
\ldots \ldots\end{array}$ & $\begin{array}{l}\text { 1. TIME }=44+32 * \mathrm{~A}+19 * \mathrm{~B} \\
\text { where } \\
\mathrm{A}=\text { Number of used channels } \\
\mathrm{B}=\text { Number of unused channels in the } \\
\text { selected block } \\
\text { 2. If any channel is given a value greater than } \\
99.99 \text { ( } 17777 \text { oCTAL) an error counter is incre- } \\
\text { mented and that } \mathrm{D} \text { to A channel is set to } \pm 99.99 \text {. } \\
\text { Upon return to the calling program } \\
\mathrm{AC}=\text { No. of overload channels } \\
\text { 3. } \mathrm{N}=\text { First channel of block } \\
\mathrm{M}=\text { Last channel of block } \\
\text { 4. A SKP in the calling sequence can be used to } \\
\text { skip a channel. In the calling sequence given } \\
\text { channel N+2 is skipped. Channel M-1 is used to } \\
\text { transfer }-25 \text { volts to analog computer. }\end{array}$ \\
\hline $\begin{array}{l}\text { DETERMINE } \\
\text { ANALOG } \\
\text { COMPUTER } \\
\text { MODE }\end{array}$ & AMODE $\$$ & $\begin{array}{l}\text { JMS AMODE } \\
\ldots \ldots\end{array}$ & $\begin{array}{l}\text { 1. TIME }=18-28 \text { ssec } \\
\text { 2. The AC at exit and the memory location \$MODE } \\
\text { are set to one of the following values } \\
0 \text { if POT set } \\
1 \text { if Hold } \\
2 \text { if Standby } \\
3 \text { if I.C. } \\
4 \text { if compute } \\
\text { 3. AMODE should be used with all mode sensitive } \\
\text { subroutines (integrator, time-delay, etc.) if } \\
\text { the digital mode is controlled by the analog } \\
\text { mode } \\
\text { 4. After JMS AMODE\$ has been used, the program may } \\
\text { use LAC \$MODE }\end{array}$ \\
\hline $\begin{array}{l}\text { SET ANALOG } \\
\text { MODE }\end{array}$ & SMODE\$ & $\begin{array}{l}\text { LAC X } \\
\text { JMS SMODES } \\
\ldots \ldots\end{array}$ & $\begin{array}{l}\text { 1. TIME }=45 \mu \mathrm{sec} \\
\text { 2. } \mathrm{X}=\text { desired mode of compute (comment 2-AMODE\$) } \\
\text { 3. This routine also sets the memory location \$MODE } \\
\text { 4. This routine is useful for controlling the } \\
\text { analog computer rep-op-rate } \\
\text { 5. If } X \text { is not } \emptyset \text { through } 4 \text { the computer mode is not } \\
\text { changed, the link is set and \$MODE is not set to } \\
\text { new value }\end{array}$ \\
\hline
\end{tabular}




\section{LIST OF SIMPL-1 FUNCTIONS (contd)}

\begin{tabular}{lll} 
FUNCTION & $\begin{array}{c}\text { SYMBOLIC } \\
\text { NAME }\end{array}$ & $\begin{array}{l}\text { CALLING } \\
\text { SEQUENCE }\end{array}$ \\
\hline
\end{tabular}

REMARKS

\section{NUMBER CONVERSION}

INTEGER BCD TO BINARY CONVERSION

VOLTAGE BCD TO VOLTAGE BINARY CONVERSION

INTEGER BINARY

TO $\mathrm{BCD}(1248)$

CONVERSION

BINARY VOLTAGE

TO BCD (1248

CONVERSION
IDBC\$

LAC $X$ JMS IDBCS

-...

1. TIME $=16-20 \mu \mathrm{sec}$

2. Converts four digit unsigned BCD digits (1248) to binary intigers

3. Example use: POT settings (sent through the interface) from the analog computer are in integer $\mathrm{BCD}$. If it were desired to make a digital computer calculation on this POT setting, the BCD number must first be converted to binary

4. $X=B C D$ NUMBER

5. $A C=$ Binary equivalent of $B C D$ NUMBER

VDBC\$ LAC $\mathrm{X}$

JMS VDBC\$ 2. Converts interface $B C D$ voltage to voltage

-.. binary

3. BIT $\emptyset$ is used for sign ( 1 = negative)

4. BIT 1 is for decimal point

If $B I T 1=1$ the number is $\mathrm{xxx} \cdot \mathrm{x}$

If BIT $1=0$ the number is $x x \cdot x x$

5. Example use:

Amplifier voltages read into digital computer from digital voltmeter are in voltage $B C D$. This routine converts the BCD number to a digital voltage for processing with normal hybrid routines

6. $A C=$ BINARY VOLTAGE

IBDC\$ LAC X 1. TIME - $90 \mu \mathrm{sec}$

JMS IBDC\$ 2. Input binary number must be positive and less .....(error) than 1000

.... (Okay) 3. If there 10 an input error the return point follows JMS IBDCS on the listing. The binary integer is not converted $\mathrm{AC}=$ Binary integer. If there is no input error the return point is the second instruction following JMS IBDC\$ $\mathrm{AC}=\mathrm{BCD}$ NUMBER

4. Example of use:

Once a POT setting is calculated by the digital computer its value must be converted to $B C D$ for transfer through the interface to the analog servo set POT system

VBDC\$

LAC $\mathrm{X}$

1. TIME $=90 \mu \mathrm{sec}$

JMS VBDC\$ ..... (error) ..... (Okay)

2. Input (binary) voltage must be positive and less than 100.00 volts

3. If there is an input error the return point follows JMS VBDCS on the listing. The voltage is not converted $A C=$ BINARY VOLTAGE

If there is no input error the return point is the second instruction following JMS VBDC\$ $\mathrm{AC}=\mathrm{BDC}$ NUMBER 


\section{LIST OF SIMPL-1 FUNCTIONS (contd)}

\begin{tabular}{lll} 
FUNCTION & $\begin{array}{c}\text { SYMBOLIC } \\
\text { NAME }\end{array}$ & CALLING \\
\hline
\end{tabular}

REMARKS

\section{TELETYPE OUTPUT LIBRARY}

A. INITIALIZATION

a. API IATYP\$

b. NOW INTERRUPT ITYP\$

B. ACCUMULATOR PRINTING
a. OCTAL
1. Signed
TOCTS\$
2. Unsigned TOCT\$
b. DECIMAL
1. Signed TDECS\$
2. Unsigned TDEC\$
c. VOLTAGE TVOLT\$
d. BINARY CODED
DECIMAL (BCD)
1. BCD Voltage TBCDV\$

2. BCD INTEGER TBCDP\$ (РOT)

3. BCD INTEGER TBCDI\$

e. ASCII CHARACTERS

1. API Typing ATYP\$ JMS ATYP\$

2. Non-Interrupt TYP\$ JMS TYP\$

3. TRIM T3T\$ JMS T3T\$

LAW TBUF

LMQ

LAW TBUFE

JMS ITYPS

JMS TOCT\$
JMS IATYP\$

JMS TOCTS\$

Left adjusted and leading zeros are suppressed

Left adjusted and leading zeros are suppressed

JMS TDECS\$ Left adjusted and leading zeros are suppressed JMS TDECS Left adjusted and leading zeros are suppressed JMS TVOLT\$

JMS TBCDV\$

JMS TBCDP\$

JMS TBCDI\$

1. Input is interface $B C D$ integer

2. All four digits are typed

1. Input is four digits of $1,2,4,8 \mathrm{BCD}$

2. Left zeros are suppressed

1. ASCII character in the $A C$ is stored in the buffer and is typed when the teleprinter is ready. If the buffer is full, the program will loop until there is an open location

1. Has an automatic time out of one second so hardware initialization is not necessary

1. Words are coded by the hybrid assembler PSEUDO - Instruction "TRIM"

2. Each character has six bits 


\section{LIST OF SIMPL-1 FUNCTIONS (contd)}

\section{FUNCTION}

SYMBOLIC

CALLING

NAME

SEQUENCE

REMARKS

C. SPACING PROGRAMS
a. Carriage return
TCR\$
JMS TCR\$
and line feed
b. Teletype space
c. Form feed
TSP\$
TFRMS
JMS TSPS
d. Horizontal
TTAB\$
JMS TFRMS
tabulation
JMS TTAB\$
D. MESSAGE PRINT
TMSG\$
LAW TAG
JMS TMSG\$

E. DEVICE TRANSFER

TTYP\$ LAW TYPS DAC TTYP\$

1. Every message must have a tag before the PSEUDO instruction TEXT. (See explanation of TEXT given in Appendix IV on pseudo instructions.)

2. Messages can be placed

a. At the end of the main program

b. At the end of subroutines

c. As a separate subroutine

1. Any of the teletype output programs (TMSG\$, TVOLT\$, etc.) can use other output devices by setting the location of the output subroutine into the location TTYP\$ (1.e.) LAW PUNCH DAC TTYP\$

Would result in a punched paper tape output

2. JMS I TTYP\$ can be used instead of JMS ATYP\$ for printing

9. SPECIAL NAMES \& STORAGE LOCATIONS

CLOCK FREQUENCY \$FC

TIME SCALE

\$TS

CLOCK

$\$ C L 1$

CLOCK

MODE STORAGE

DVM CHANNEL SELECTOR

$\$ C L 2$
\$MODE

\begin{tabular}{lrl} 
\$MXDVM & 1. Contains instruct \\
(SPECIAL NAMES USING AUTO INDEX REGISTER) \\
\hline
\end{tabular}

1. Used by time dependent programs

2. Normally $5 \emptyset \emptyset \emptyset^{\prime}$ or $\left(1161 \emptyset_{8}\right)$ but can be changed by user

1. Used by time dependent programs

2. Normally 1 but can be any number greater than 1 (see comments for ICL\$)
FGI\$

\$INTI

\$TDI
1. Name associated with auto index register 17 and is used by function generator programs

2. Name associated with auto index register 16 and is used by the integration subroutine RINT\$

1. Name associated with auto index register 15 and is used by the time delay program TDI\$

(Auto index registers 10 thru 14 are available to the user)

\section{(SPECIAL STORAGE LOCATIONS)}

Memory locations 40 thru 57 are reserved as return points for API interrupts (see Appendix VI). Some hybrid programs have been clobbered by spurious API pulses when these locations have been used in the regular program. Therefore, its atvisable to put HALTS in all unused API channel locations. 
LIST OF SIMPL-1 FUNCTIONS (contd)

\section{FUNCTION}

10. CARD READER

READ A CARD

\section{SYMBOLIC CALLING}

NAME SEQUENCE

CARD\$

CONVCA\$ $=10$

CARDB\$ $=11$

JMS CARD\$

JMS CNVCD\$

or JMS CONVT\$
1. Must select an auto index register for CARDB\$ (and CNVCA\$ if CNVCD\$ is used for conversion from IBM code to ASCII).

2. The calling sequence given will read a card and convert the IBM characters to ASCII. If IBM characters are desired use:

$$
\begin{aligned}
& \text { CARDB } \$=10 \\
& \text { JMS CARD\$ }
\end{aligned}
$$

3. The information contained in the card buffer (CBUF\$ thru CBUF\$ + 110) will be lost unless it is transferred out before another card is read.

4. If a CAF is used in the main program, the card reader must be made ready after the program has been started.

5. Subroutine CARD\$ disables the API. It is enabled before returning to the main program.

6. CNVTC $\$$ converts the contents of the card buffer to ASCII, character for character in the same buffer. If a character cannot be located in the conversion table a backward slash is put in its place.

7. CNVCD\$ performs al1 operations of CNVTC $\$$ and converts two or more spaces on a card with a carriage return and line feed. (A blank card is interpeted as a carriage return and a line feed.

\section{SPECIAL KEYPUNCH CHARACTERS :}

Conflicting characters from one keypunch to another, as well as "invisible" characters, have made several special codes necessary.

\#⿰ $\quad 3$ over 4 punch

! 1 over 4 punch

"FORM" 1 over 2 over 4 punch

or "END OF PAGE"

The keys on some machines are imprinted with a character other than that which is desired.

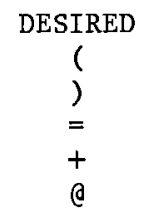

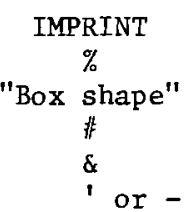

(not the regular minus) 


\section{LIST OF SIMPL-1 FUNCTIONS (contd)}

$\begin{array}{lll}\text { FUNCTION } & \begin{array}{c}\text { SYMBOLIC } \\ \text { NAME }\end{array} & \text { CALLING } \\ \text { SEQUENCE }\end{array}$

REMARKS

11. SET POTS AND READ ANALOG COMPONENTS UNDER CONTROL OF USERS PROGRAM

SET POTS ON THE

ANALOG WITHIN

USERS PROGRAM
TO READ ANALOG COMPONENTS AND STORE THE READINGS IN A TABLE AND PRINT THE READINGS IF DESIRED. (This can be accomplished within the users program)
PSET\$

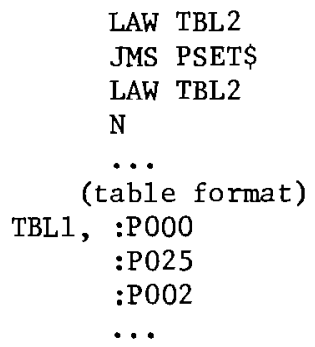

TBL 2, 9.99

10.00

6.23

....
1. TBL1 contains the interface codes for the pot addresses. TBL2 contains the corresponding pot settings. $\mathrm{N}$ is the number of pots to be set.

2. The program requires the use of one multiplexer line. The line normally selected is MX23 and the SEL hole on the analog must be patched to MX23. If a different MX channe1 is desired then it must be patched to the SEL hole and the following sequence must preceed the use of PSET\$

\section{LAC (MXN DAC \$MXDVM}

where $\mathrm{N}$ is the number of the selected channel.

3. This program requires that the teletype be initialized and uses library routines TMSG\$, TTYP\$, VDBC\$, IDBC\$.

4. Pots can also be set by using the POT COEFFICIENT EDITOR described in Appendix VII, but this method must be used external to the users program.

1. TIME $=$ usec per component (when printout is not requested.)

2. TBL1 contains a list of analog component addresses and TBL2 is a table where the readings are stored. $\mathrm{N}$ is the number of components to be read.

3. The program will list the components and their readings if the link is set when the program is called or it will simply read and store the values if the link is cleared.

4. This program requires the use of a multiplexer line and it must be patched to the SEL hole. The channel normally selected for use is channel 23 , but any channel $N$ may be selected if it is patched to the SEL hole and the following sequence preceeds the use of READ\$

5. This program requires that the teletype be initialized and uses the library routines TTYP\$, TMSG\$, TCR\$, TTAB\$, and TVOLT\$.

6. The READ POTS program and POT EDITOR program described in Appendix VII can also be used for reading pots, but these programs must be executed external to the users program. 
APPENDIX C

HYBRID ASSEMBLER INSTRUCTIONS

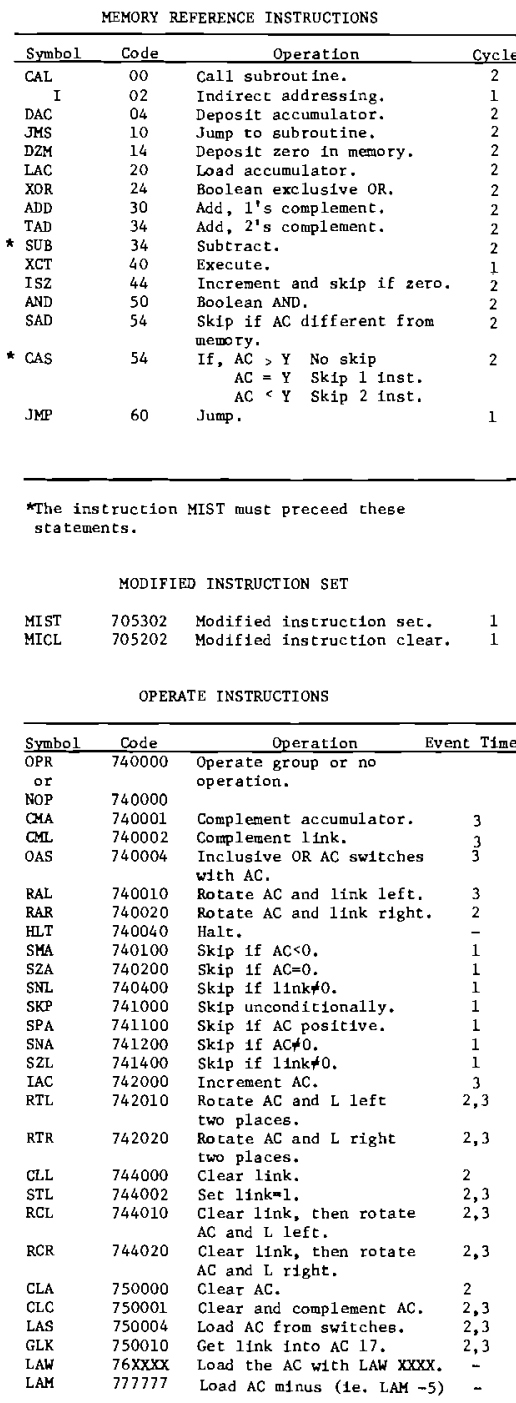

\begin{tabular}{|c|c|c|c|}
\hline & & & \\
\hline $\begin{array}{l}\frac{\text { Symbol }}{\text { EAE }} \\
\text { LRS } \\
\text { LRSS }\end{array}$ & $\begin{array}{l}\text { Code } \\
640000 \\
640550 \\
665000\end{array}$ & $\begin{array}{l}\text { Operation } \\
\text { No operat1on. } \\
\text { Long right sh1ft. } \\
\text { Long right shift, }\end{array}$ & $\begin{array}{l}\frac{T \text { Ime }(\mathrm{sec})}{1.75} \\
1.6+0.1 \mathrm{n} \\
1.6+0.1 \mathrm{n}\end{array}$ \\
\hline $\begin{array}{l}\text { LLS } \\
\text { LLSS }\end{array}$ & $\begin{array}{l}640600 \\
660600\end{array}$ & $\begin{array}{l}\text { s1gned. } \\
\text { Long left shift. } \\
\text { Long left } \mathrm{shHft} \text {, }\end{array}$ & $\begin{array}{l}1.6+0.1 \pi \\
1.6+0.1 n\end{array}$ \\
\hline AIS & $\begin{array}{l}640700 \\
660700\end{array}$ & $\begin{array}{l}\text { signed. } \\
\text { Accunulator left shift. } \\
\text { Accurulator left shift, }\end{array}$ & $\begin{array}{l}.1 .6+0.1 \mathrm{n} \\
, 1.6+0.1 \mathrm{n}\end{array}$ \\
\hline $\begin{array}{l}\text { NORY } \\
\text { OAMO }\end{array}$ & $\begin{array}{l}640444 \\
642000\end{array}$ & $\begin{array}{l}\text { Ellnged. } \\
\text { No trmalize, unsigned. } \\
\text { OR MQ+AC, Results ing in }\end{array}$ & $\begin{array}{l}2.4+0.35 n+0.2 n \\
1.75\end{array}$ \\
\hline $\begin{array}{l}\text { NoRMS } \\
\text { MUL } \\
\text { MULS } \\
\text { DIV } \\
\text { DIV }\end{array}$ & $\begin{array}{l}660444 \\
653122 \\
657122 \\
6412323 \\
644323\end{array}$ & 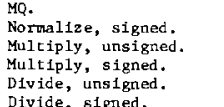 & $\begin{array}{l}1.6+0.1 \mathrm{n} \\
1.6+0.1 \mathrm{n} \\
2.4+0.1 \mathrm{n}+0.25 \mathrm{~m} \\
2.4+0.1 \mathrm{n}+0.25 \mathrm{~m}\end{array}$ \\
\hline
\end{tabular}

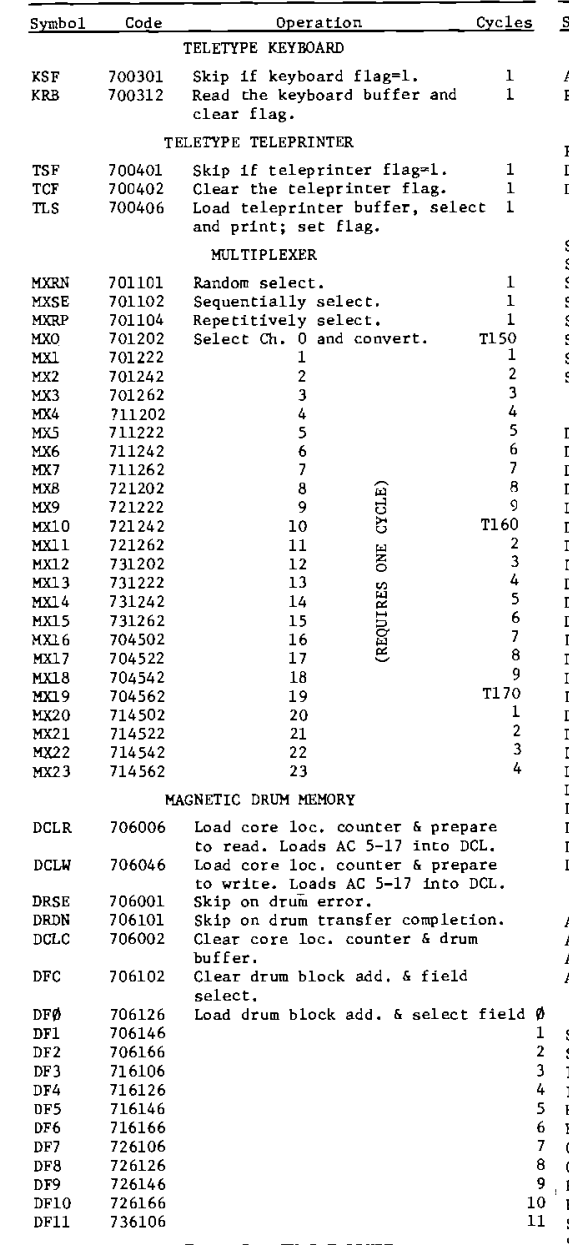

\begin{tabular}{lccc}
\hline Symbo1 & Code & Operation & Cyc1es \\
& DIGITAL VOLT METER &
\end{tabular}

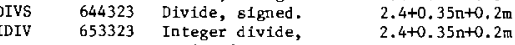

$\begin{array}{llll}\text { IDTVS } & 657323 & \begin{array}{l}\text { unsigned. } \\ \text { Inceger divide, signed. }\end{array} & 2.4+0.35 n+0.2 \mathrm{~m} \\ \text { FRDIV } & 650323 & \text { Fraction divide, } & 2.4+0.35 n+0.2 \mathrm{~m}\end{array}$

FRDIVS $654323 \quad \begin{aligned} & \text { unsigned. } \\ & \text { Fraction divide, }\end{aligned} \quad 2.4+0.35 n+0.2 \mathrm{~m}$

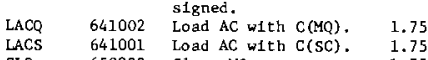

$\begin{array}{llll}\text { LACS } & 641001 & \text { Lad AC w1th C(SC). } & 1.75 \\ \text { CLO } & 650000 & \text { Clear Mo. } \\ \text { ABS } & 644000 & \text { Take absolute value } & 1.75 \\ & & 1.75\end{array}$

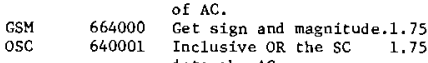

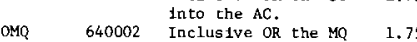

\begin{tabular}{llll} 
CMQ & 640004 & $\begin{array}{l}\text { Into the AC. } \\
\text { Conplemenc che MQ. }\end{array}$ & 1.75 \\
LMQ & 652000 & Load MQ with CAC). & 1.75 \\
\hline
\end{tabular}

$n=$ shlft count
$m=$ one bits $1 \mathrm{n}$ multiplier

INPUT/OUTPUT TRANSFER INSTRUCTIONS

$\underset{\text { Symbol Code }}{\text { Operatton }}$

Interrupt off. Disable the PIC.
Interrupt on. Enable the PIC.

PERFORATED TAPE READER

$\begin{array}{lll}\text { RSF } & 700101 & \text { SkL1p if reader flag=1. } \\ \text { RCF } & 700102 & \text { Inclustve OR reader buffer }\end{array}$

RRB $700112 \begin{aligned} & \text { Into AC and clear flag. } \\ & \text { Read reader buffer and c1 ear }\end{aligned}$

$\begin{array}{lll}\text { RSA } & 700104 & \begin{array}{l}\text { reader } \\ \text { Reader in alphenumer ic. } \\ \text { RSB }\end{array} \quad 700144 \\ \text { Reader in binary. }\end{array}$

PERFORATED tAPE PUNCH

$\begin{array}{lll}\text { PSF } & 700201 & \text { Skip 1f punch flag }=1 \\ \text { PCF } & 70202 & \text { Clear punch flag. } \\ \text { PSA or } & 700204 & \text { Punch alphenumeric. } \\ & & \end{array}$

$\begin{array}{lll}\text { PLS } & 700206 & \\ \text { PSB } & 700244 & \text { Punch binary. }\end{array}$

DECtape SYSTEM

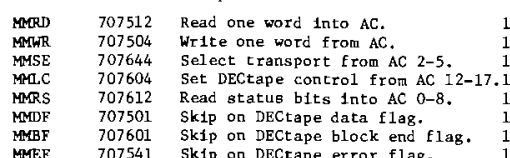
CAC 705501 Clear al1 channels.

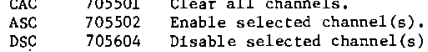
$\begin{array}{lll} & & \text { I/O EQUIPMENT } \\ \text { IORS } 700314 & \text { Input/output read status. } \\ \text { Skip } 1 \mathrm{f} \text { KSR } 33 \text { is used. }\end{array}$

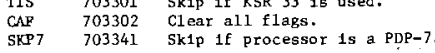

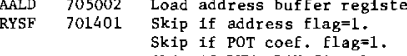

Skip if DVM RDBK flagel.
Poad POT coef, buf fer reg1s

DVMR 701404 DVM read and load buffer reg.

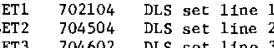

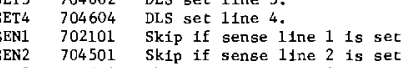

$\begin{array}{lll}\text { SEN3 } & 704601 & \text { Skip if senbe line } 3 \text { is set. } \\ \text { SEN4 } & 70 \times 001 & \text { Skip if sense 11ne } 41 \mathrm{~s} \text { sec. }\end{array}$

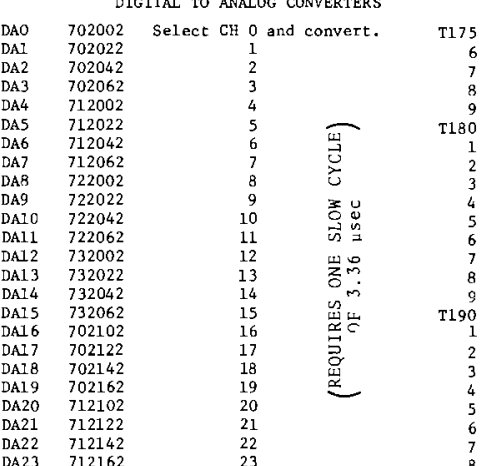

$\begin{array}{lll}\text { DA221 } & 712122 & 22 \\ \text { DA223 } & 712142 & 22 \\ \text { DA12162 } & 23\end{array}$

aNaLOG to digital CONVERTER

$\begin{array}{lll}\text { ADOV } & 701201 & \text { Sk1p 1f overload. } \\ \text { ADSF } & 701301 & \text { Skip if A } A \text { D flag set. }\end{array}$

ADCV 701304 Convert.

ANALOG COMPUTER MODE SET AND SENSE

SBSF 702001 Skip if standby f1ag is set.

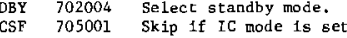

$\begin{array}{lll}\text { HDSF } & 705004 & \text { Select IC mode. } \\ \text { SkIP if hold mode 1s set. }\end{array}$

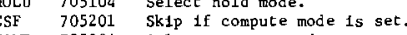

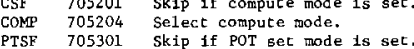

Skip if Por set mode $1 \mathrm{~s}$
Select POT set mode.

$\begin{array}{lll}\text { SERF } & 701501 & \text { Sk1p If servo flag is } \\ \text { SERV } & 701504 & \text { POT servo operate. }\end{array}$

$\begin{array}{llll}\text { CRSD } & 706401 & \text { Sk1p if card reader 1s done. } \\ \text { CRSF } & 706501 & \text { Skip If card reader is ready. }\end{array}$

$\begin{array}{llll}\text { CRR } & 706504 & \begin{array}{l}\text { Read cards. } \\ \text { CBSF }\end{array} 706601 & \text { Sk1p if card reader buffer }\end{array}$

CRBS $706602 \quad \begin{aligned} & \text { 1s loaded. } \\ & \text { Shift card reader buffer into }\end{aligned}$

CRBC 706604 AC one shlft.

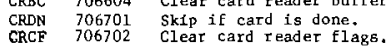


APPENDIX D

\section{RADIX CONTROL}

DECIMAL A11 numbers not imbedded in symbols can be interpreted as decinal or octal respectively The intial mote is A appost rophy (') wili be considered to be antaining an be a decimal conor DECIMAL. (' $\left.1 \emptyset \emptyset=1 \emptyset \emptyset_{10}=144_{8}\right)$.

VOLTS

A series of digits containing a decimal point (.) is interpreted as a voltage as long as the decimal point is preceeded by a digit $(25.00=40008)$.

RATIO A series of digits contalning a decimal point is interpreted as a ratio if the pseudo instruction RATIO is
given before the number, under RATIO Control $\emptyset .25=4 \emptyset \emptyset_{8}$. If the pseudo fnstruction vOLTS is used the assembler (decimal point) mode is returned to voltage.

A series of characters preceded by a colon (:) is converted from a $8,4,2,1 \mathrm{BCD}$ sequence to its octal equivalent. Examples of the two forms are:

1. BCD POT Coefficient $(: 3805=\emptyset 340058)$

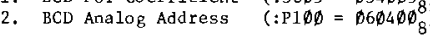

\section{TABLE FORMATION}

SHIFT

Causes the word preceding SHIFT to be rotated left nine binary positions and masked with 777000 , leaving the engt half blank. Sill is useful in forming double entry tables, tables whth one value stored in the leftbits.

\section{SYMBOL TABLE CONTROL}

EXPUNGE Removes all symbols (except pseudo-instructions) from the Assembler's symbol table.

FIX

Resets the symbol table so that all currently defined symbols are part of the permanent symbol table. (This instruction overrides the AcS optton to restore at assembly time.)

\section{VARIABLE CONTROL}

VARIABLES Places all currently defined variables at addresses beginning with the address indicated by the location counter before processing of the program continues.

BAR N

Allows $N$ registers for each variable containing the character $?$ (see page 10).

\section{MULTIPLICATION CONTROL}

NORMUL

This is the normal multiplication node. It causes the answer from a " $\star$ " multiplication to be taken from the the 36 bit product.)

MODMUL

This causes the answer from a " $"$ "multiplication to be cant bits of the 36 bit product.)

\section{PUNCH CONTROL}

PUNDEF SYMB1, Punches definitions of the listed symbols in Assembler SYMB2, SYMB3 format.

SYMBOLS

Punches ensuing symbols in Assembler format.

NOSYMBOLS

Stops the punching of Assembler symbols and restores the mode set by ACS concerning DDT symbols.

PUNCH A Punches the value of the expression A in binary at this time.

(See pages 25-26 for a more detailed explanation)

\section{END OF PROGRAM}

NOINPUT

Suppresses purching of the loader--punches a JMP 17600 , the starting address of the FF Loader.

StART A Upon loading, causes the program to start at register A.

PAUSE A Upon loading, causes the computer to halt. When CONTINU is pressed, the program will start at A. (See page 17.)

* Page numbers refer to the PDP-7 Symbolic Assembler Programining Manual (DigItal-7-3-S), Digital Equipment Corporation (1965)
LINKAGE CONTROL

INTERNAL This indicates that the symbolic name is a tag internal to the Sub-program example:

INTERNAL CONV
CONV,

INTERNAL is used to establish address definition for the linking

In a sub-program, a tag containing a dollar sign ( $\$$ ) may be used to eliminate the INTERNAL pseudo-instruction.

$$
\text { Examp1e: } \quad \$ C O N V \text { LAC } X
$$

EXTERNAL This indicates that the symbolic name or names following are external to the main program or sub-program being assembled. in question are mentioned in the symbolic listing.

Example:

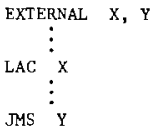

The EXTERNAL pseuod-instruction completes the comnunication linkage necessary for the loader to load user sub-programs and library sub-routines.

Any external tag containing a dollar sign ( $\$$ ) need not be mentioned in an EXTERNAL pseudo-instruction.

$$
\text { Example: JMP } \$ \text { CONV }
$$

LIBFRM This is used following a sub-program to Indicate that the progran is to be punched in a special library format. All the librar library The following the instructions for preparing a library tape. LIBFPM pseudo-instruction all the tags must have been referred to by an INTEnNa pseudo

Example:

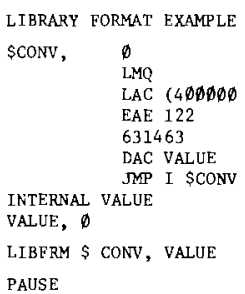

In this example, a library format entry for \$CON and VALUE wil be punched at the front of the 11brary tape. If either \$CONV or must have been used), the linking loader would load the entire sub-program in the example into memory.

\section{TEXT HANDLING}

The following pseudo-instructions to the Hybrid Assembler can be used to generate character and text codes in a program:

CHAR

This instruction causes the ASC II code of the next ron-space character to be taken as a numeric value (LAF CHAR = LAW 260)

This instruction causes the characters following the "space" after TRIM and preceding a termination character (carriage return or tab) to be placed in memory as ASC II trim codes.

$\begin{array}{rlc}\text { TRIM CODE } & \text { ASC II CODE } & \frac{\text { CHARACTER }}{\text { none }} \\ 1 \text { to } 32 & 3 \emptyset 1 \text { to } 332 & \text { A to } 2 \\ 33 \text { to } 37 & 333 \text { to } 337 & \text { [, , e etc. } \\ 40 \text { to } 77 & 24 \emptyset \text { to } 277 & \text { space, } \because, " \text {, etc. }\end{array}$

Example: HEART, TRIM HAVE A HEART:

is identical to

HEART, $10 \$ 126$

54001

401005

$410 \emptyset \emptyset 0$

A subroutine, such as, T3T $\$$ in the teletype output library can be used to print a ASC II trim coded word.

The pseudo-instruction "TEXT" causes text trim codes to be placed into memory. The text starts after the space, tab, or carrlage
return following TEXT and continues until a (a) symbol is Any ASC II character except TEXT coded message.

Example:

DOG, TEXT MY DOG HAS FLEAS, (a) will place the text trim codes for "MY DOG HAS FLEA" into nemory starting at symbolic address DOG.

A subroutine, such as, TMSG\$ in the teletype output library should 


\title{
ASSEMBLER PSEUDO-INSTRUCTIONS (contd)
}

\author{
Use of PSEUDO-instruction LIBFRM For Preparing a User LIBRARY Format Tape
}

The "library format" pseudo-instruction, LIBFRM, is used following a sub-program to indicate that the program is to be punched in a special library format. All the library tapes are of this format. The user can crete h1s own library tape by following the instructions for preparing a library tape. The linking loader w1ll selectively load these programs. A LIBFRM pseudo-instruction may have any number of arguments but all the tags must have been referred to by an INTERNAL pseudo-instruction or must contain $\$$.

Example:

LIBRARY FORMAT EXAMPLE

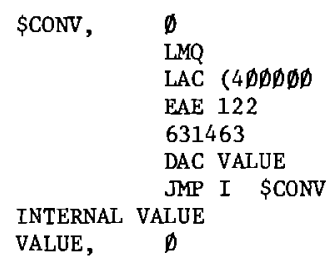

LIBFRM \$CONV, VALUE

PAUSE

In this example, a library format entry for $\$$ CONV and VALUE will be punched at the front of the library tape. If either \$CONV or VALUE or both are called for in the main program (EXTERNAL VALUE must have been used), the linking loader would load

the entire sub-program in the example into memory.

\section{Preparing a Library Tape}

A library format tape should be created as follows:

1. Terminate all the sub-program symbolic listings with a LIBFRM pseudo-instruction.

2. Select the proper ordering of the sub-program symbolic tapes so that sub-routines that are called by other library sub-routines come last.

3. Assemble the programs in reverse order to that desired. (Assembler output tape comes out of punch backwards.)

4. If a readable title is desired, assemble a dummy title maker as the first tape of the last program assembled.

5. The AC switches should be used to perform these functions:

\begin{tabular}{|c|c|c|}
\hline$\underline{\mathrm{ACS}}$ & POSITION & FUNCTION \\
\hline$\emptyset$ & $\begin{array}{l}\text { up : } \\
\text { down : }\end{array}$ & $\begin{array}{l}\text { Look at switches } 1-4 \\
\text { Ignore switches } 1-4\end{array}$ \\
\hline 1 & $\begin{array}{l}\text { up: } \\
\text { down : }\end{array}$ & $\begin{array}{l}\text { Suppress punching } \\
\text { Punch }\end{array}$ \\
\hline 2 & $\begin{array}{l}\text { up: } \\
\text { down: }\end{array}$ & $\begin{array}{l}\text { Suppress DDT symbols } \\
\text { Punch DDT symbols }\end{array}$ \\
\hline 3 & $\begin{array}{l}\text { up: } \\
\text { down: }\end{array}$ & $\begin{array}{l}\text { Take this t1tle on this tape } \\
\text { Don't take title on this tape }\end{array}$ \\
\hline 4 & $\begin{array}{l}\text { up : } \\
\text { down : }\end{array}$ & $\begin{array}{l}\text { Restore assembler } \\
\text { Don't restore assembler }\end{array}$ \\
\hline 13 & $\begin{array}{l}\text { up: } \\
\text { down : }\end{array}$ & $\begin{array}{l}\text { Suppress readable title on } \\
\text { library format tape } \\
\text { Punch readable title on } \\
\text { library format tape. }\end{array}$ \\
\hline 14 & $\begin{array}{l}\text { up: } \\
\text { down: }\end{array}$ & $\begin{array}{l}\text { Type spaces for tabs } \\
\text { Type automatic tab }\end{array}$ \\
\hline 15 & down: & $\begin{array}{l}\text { Restore symbol table to permanent } \\
\text { symbols (after symbol printouts } \\
\text { if requested). Starting the next } \\
\text { assembly with conTINUE has the same } \\
\text { effect. } \\
\text { No action taken }\end{array}$ \\
\hline 16 & $\begin{array}{l}\text { up: } \\
\text { down: }\end{array}$ & $\begin{array}{l}\text { Symbol printout, numerical order } \\
\text { No action taken }\end{array}$ \\
\hline 17 & $\begin{array}{l}\text { up: } \\
\text { down: }\end{array}$ & $\begin{array}{l}\text { Symbol printout, alphabetical order } \\
\text { No action taken }\end{array}$ \\
\hline
\end{tabular}

On library tapes, it is desirable to suppress the readable titles of the individual programs so as to shorten the tape. It is also desirable to suppress symbol punching for DDT so as to save space in DDT's symbol table.

NOTE: DDT symbols for "internal tags" are punched regardless of the condition of the AC switches. 
APPENDIX E

AUTOMATIC PRIORITY INTERRUPT INSTRUCTIONS AND EXAMPLES

API CHANNEL FLAG ASSIGNMENTS

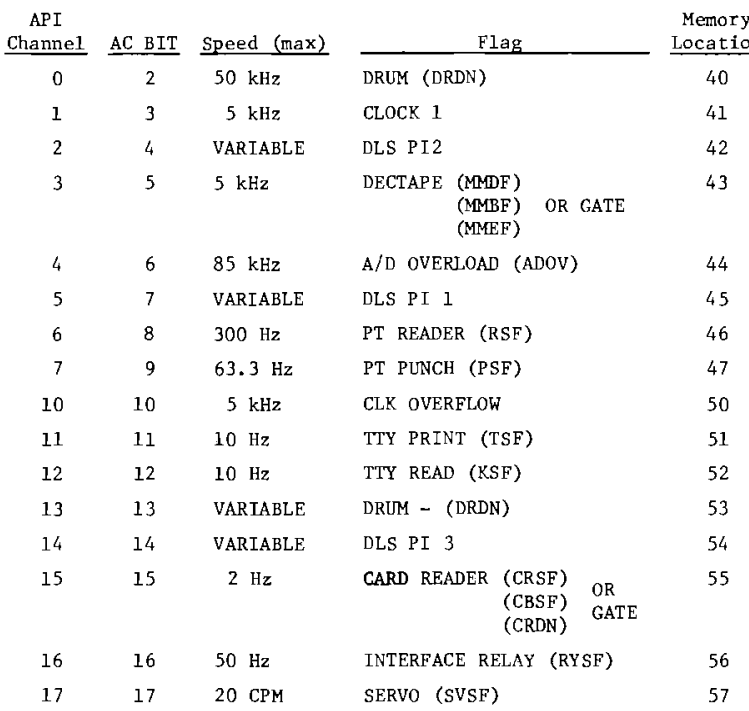

EXAMPLE 1

From the table above CLOCK 1 and CLK OVERFLOW (API channels 1 and 10 ) are initialized by micro programming AC BIT 3 and 10 .

\begin{tabular}{|rrr|rrr|rrr|rrr|rrr|rrr|}
\hline 0 & 1 & 2 & 3 & 4 & 5 & 6 & 7 & 8 & 9 & 10 & 11 & 12 & 13 & 14 & 15 & 16 & 17 \\
\hline 0 & 0 & 0 & 1 & 0 & 0 & 0 & 0 & 0 & 0 & 1 & 0 & 0 & 0 & 0 & 0 & 0 & 0
\end{tabular}

The corresponding octal number is 040200 . The programming sequence required to initialize API channels 1 and 10 is:

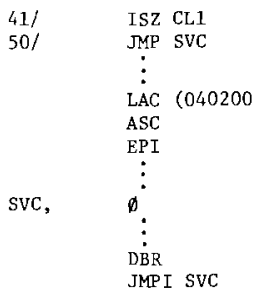

Every $1 / 5000$ second a data break executes the instruction in location 41. Which in this example is ISZ CLl. (A clock service routine usually reinitializes CLl to a negative number) Every $1 / 5000$ cond the value of $\mathrm{CLl}$ is incremented uncil its value passes thru 777777 to At this point API channel 10 transfers control thru 777778 to 0 . At this point API channel 10 transfers control to location 50 (which is us it a jus to a service routine). If a service routine is used it should first store the contents of $A C, M Q, P C$, and link before proceeding with incerrupt calculations (such as, integration or time delay). After interrupt calculation are performed the value of CLl should be reinitialized to a nega tive number and the values of the $\mathrm{AC}, \mathrm{MQ}, \mathrm{PC}$ and link restored.

The SIMPLI subroutines ICL $\$$ and RCL $\$$ can be used to automatIcally perform the above operations. (However, ICL\$ and RCL $\$$ require more machine time than generally required with assembly language.

Example 2 illustrates in detail how a API could be used for periodic conversion of $\mathrm{A} / \mathrm{D}$ channel 14 .
EXAMPLE 2. PERIODIC A/D CONVERSION OF CHANNEL 14 USINF THE API AND $5 \mathrm{kHz}$ CLOCK

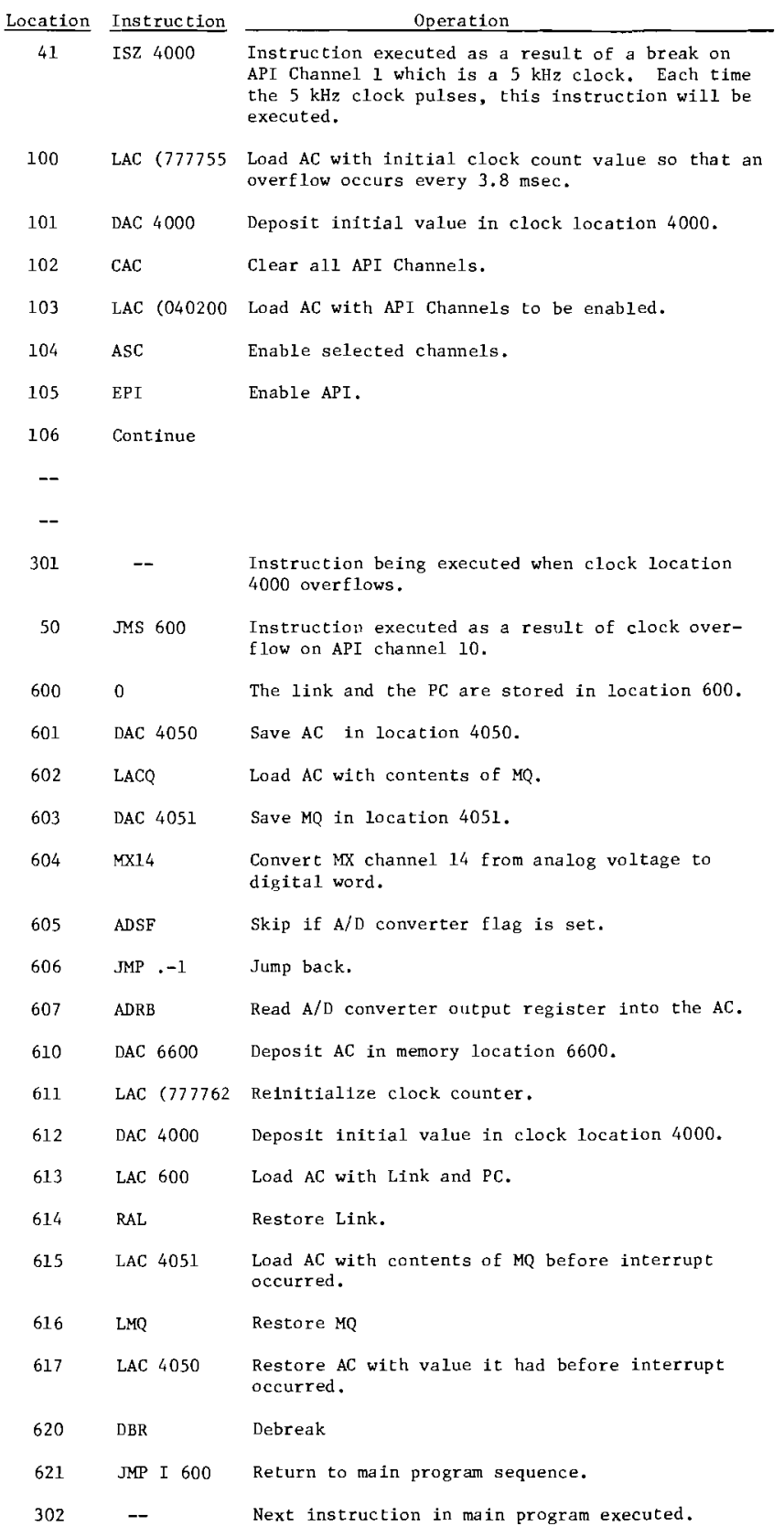


APPENDIX F

SIMPL-I SYSTEM PROGRAM INDEX

BLOCK

$\underline{\text { TITLE }}$

APPLICATION

DECTAPE INPUT/OUTPUT

D. T. (HIGH)READER

D. T. (RIM, FF) WRITER

D.T. (CORE)WRITER

D.T. (DT COPY)FAST COPIER

D.T. SELECTIVE COPIER

D.T. OCTAL PRINT

DECSYS BOOTSTRAP

ZERO BLOCK FINDER

DECTOG

READ A PROGRAM INTO MEMORY

TRANSFER RIM \& FF TO MAG TAPE

TRANSFER FROM CORE TO MAG TAPE

COPY MAG TAPES

COPY SELECTED BLOCKS TO NEW MAG TAPE

PRINT CONTENT OF MAG TAPE IN OCTAL

USED TO LOAD DECSYS-7 FORTRAN II PROBLEMS

LISTS ALL BLOCKS OF LOCATIONS CONTAINING

ZERO

DECTAPE FORMAT GENERATOR

\section{PAPER TAPE INPUT/OUTPUT}

LOW R.I. PUNCH

HIGH R.I. PUNCH

LOW RIM PUNCH

HIGH RIM PUNCH

TAPE (PAPER) REPRODUCER

F.F. OCTAL PRINT

LIBDUB

SYMBOLIC TAPE LISTER
PUNCH READ IN MODE PROGRAMS FROM CORE

PUNCH RIM MODE PROGRAM FROM CORE

REPRODUCE PAPER TAPE

USED FOR DEBUGGING FUNNY FORMAT SYSTEM

PROGRAMS (i.e., THE ASSEMBLER)

DUPLICATE HYBRID RELOCATABLE LIBRARY FORMAT

TAPE

LIST SYMBOLIC TAPE WITH PAGE NO.

\section{CARD INPUT/OUTPUT}

LIST DECK

PUNCH DECK

EDITOR

SYMBOLIC TAPE EDITOR
TYPE CONTENTS OF DECK OF CARDS PUNCH PAPER TAPE OF CONTENTS ON CARDS

\section{$\underline{\text { ASSEMBLERS }}$}

ABSOLUTE HYBRID

RELOCATABLE HYBRID ASSEMBLER EXTENDED ASSEMBLER (DEC)

\section{DEBUGGING PROGRAMS}

HYBRID DDT BASIC DDT (OPERATING INSTRUCTIONS IN DIGITAL 7-4-S)

MEMORY DUMPS

OCTAL MEMORY DUMP (PRINTER) ABSOLUTE MEMORY DUMP (PRINTER)

\section{ANALOG SETUP AND CHECKOUT}

READ POTS

PUNCH STEADY STATE CHECK TAPE

STEADY STATE CHECK

POT COEFFICIENT EDITOR
LIST POTS FROM ANALOG COMPUTER PUNCHES OUTPUT PAPER TAPE OF ANALOG COMPUTING ELEMENTS

READS PREVIOUSLY PUNCHED PAPER TAPE AND COMPARES WITH PRESENT VALUES

OPEN BLOCKS 
APPENDIX G

SIMPL-1 SYSTEMS PROGRAM OPERATING INSTRUCTIONS

DECTAPE INPUT/OUTPUT

BLOCK 1 - TO READ A PROGRAM INTO MEMORY

1. Select DECtape Unit Set AC-2 thru AC-5 to octal address of Unit

2. Select Program Block Number Set $A C-6$ thru AC-17 with (Block No.)

Selected program should be read into core memory

If not, set address switches to 7640 and depress START - the HIGH DSD reader should be read from DECtape by the LOW DSD reader some programs will destroy both readers, if this happens, use the read-in format paper tape* - this should not happen of ten

PROGRAM WILL ENTER MEMORY AND MAY BE EXECUTED

*Load read-in format tape into reader, address 17600 , and press READ-IN.

BLOCK 2 - DSD DECTAPE WRITER (RIM,FF) (TRANSFER (RIM,FE) TO MAG TAPE

1. Call writer into memory thru the DECtape reader

2. Select DECtape Unit Set AC- 2 thru AC-5 to octal address of Unit

3. Designate Block Number (where you wish your program to start)

4. Load RIM or FUNNY FORMAT tape in reader Set transport write-lock $S W$ to write

5. Set AC-0 to 1ndicate format of paper tape Set to one to indicate RIM or ZERO to indicate FUNNY FORMAT

6. If $\mathrm{AC}-0$ is one you may demand a PAUSE after readin by setting $\mathrm{AC}-1$ to $\mathrm{ONE}$

7. Depress CONTINUE

Program will be loaded and computer will HALT with the next open block number in the accumulator

BLOCK 4 - DSD DECTAPE "CORE" WRITER (TRANSFER FROM CORE TO MAG $\underline{\text { TAPE) }}$

1. Load program into memory (any method of loading)

2. Load writer (any method of loading)

3. Select DECtape Unit

Set $\mathrm{AC}-2$ thru $\mathrm{AC}-5$ to octal address of Unit

4. Designate Block Number (where you wish your

progran to start)

5. Depress CONTINUE

6. Select program starting address Set $\mathrm{AC}-5$ thru $\mathrm{AC}-17$

7. Demand PAUSE on loading if desired Set $\mathrm{AC}-0$ to ONE

8. Depress CONTINUE

9. Set first core location this block

10. Depress Continue

11. Set last core location this block

12. If this is last block set ACO to ONE

13. Depress CONTINUE

14. If ACO was ZERO program will halt with ZERO in the accumulator CONTINUE from Step 9

If ACO was ONE program will halt with the next open block in the accumulator

LOAD COMPLETE
BLOCK 5 - "DTCOPY" (DSD DECTAPE) (COPY MAG TAPES)

1. Select DECtape Unit (AC Switches 2-5)

2. Select Block Number (AC Switches 6-17)

3. Select HIGH or LOW CSE DECtape Reader (Address Switches)

4. Depress START

5. Load tape to be copied on Unit 1 (write-1ock)

6. Load blank tape on Unit 2 (write)

7. Depress CONTINUE

BLOCK 6 - DECTAPE SELECTIVE COPY (COPIES SELECTED BLOCKS TO NEW MAG TAPE)

This is a simple minded procedure which copies information from a DECtape on Unit one to a DECtape on Unit Two. Information is transferred through memory one block at a time with no comparison or sum-checking. The check-sum read from Unit one is written (right or wrong on Unit Two

USE :

1. Read selective copy routine into memory

2. Put tape to be copied on Unit One (write-lock)

3. Put blank tape on Unit Two (write)

4. Select first block to be copied (AC Switches 6-17)

5. Depress CONTINUE

6. Select last block to be copied (AC Switches 6-17)

7. Depress ConTINUE

8. Program halts at 1ocation 22 with the last block duplicated in the accumulator

9. To copy another series of blocks return to step 4

BLOCK 7 - DECTAPE OCTAL PRINT

A debugging routine which prints the octal contents of a DECtape

USE:

1. Read DECtape octal print routine into memory

2. Select DECtape Unit

Set AC Switches 2-5 to desired Unit

3. Select starting block Set AC Switches 6-17 to first block to be printed

4. Depress ConTINUE

5. Routine will print contents of successive blocks until the end of the tape is reached or halted by the operator

6. Routine may be caused to ha1t at the end of the block being printed by setting ACO to ONE

7. The routine may be restarted at Step 2 by setting the Address switches to 23 and depressing START or if it was halted by setting ACO it may be RESTARTED at Step 2 by depressing CONTINUE

BLOCK 20 - ZERO BLOCK FINDER

This routine maps the location of all zEROS from location 100 to the end of menory

The program starts automatically when loaded.

No operator intervention is necessary

BLOCK 100 - DECTOG

(See DECUs furnished program 2-A (white notebooks) for information) 


\section{PAPER TAPE INPUT/OUTPUT}

BLOCK 10 - DECSYS BOOTSTRAP

1. Read program from mag tape

2. See DECSYS-7 Operating Manual (Digital-7-5-5)

BLOCK 12 - LOW READ IN PUNCH

1. Read in program from mag tape

2. Set lower address in address switches

3. Press continuE

4. Set high address in address switches

6. See DECUS furnished program 1-R (white notebook)

See DECUS furnished program 1-R wh
for further information on program

BLOCK 13 - HIGH READ IN PUNCH

1. Same operating instructions as for Low Read In Punch (Block 12)

2. See DECUS furnished program 1-R (white notebook) for further information about program

BLOCK 14 - LOW RIM PUNCH

1. Same operating instructions as for Low Read In Punch (Block 12)

2. See DECUS furnished program 1-0 (white notebook) for further information about program

BLOCK 15 - HIGH RIM PUNCH

1. Same operating instructions as for Low Read In Punch (Block 12)

2. See DECUS furnished program 1-0 (white notebook) for further information about program

BLOCK - 16 - TAPE REPRODUCER

1. Read in program from mag tape

2. Put paper tape in reader

3. Press CONTINUE

BLOCK 17 - FF OCTAL PRINT

1. Read in program from mag tape

2. Place paper tape in reader

3. Press CONTINUE

BLOCK 24 - LIBDUH

1. Read in program from mag tape

2. Put original 1ibrary tape in reader

3. Press CONTINUE (this duplicates readable title)

4. Press CONTINUE (this reads first program into memory

and types name(s) of library program on Teletype)

5. (a) Press CONTINUE to punch what was read in durtng step 4. When computer halts go to step 4 to step 4 . When computer

(b) If deleteing a program set address switches to 100 and press START. This reads next program into memory and types program name $(s)$ on Teletype, After computer halts go to step $5 a$

(c) If changing program take old tape out of reader (be sure to mark position of tape), place new assembled tape in reader, set address switches to 100 , and press START. This reads new program into memory and types program name(s) on Teletype. After computer HaLTS go to step $5 a$.

BLOCK 42 - SYMBOLIC TAPE LISTER

1. Read in program from mag tape

2. Put paper tape in reader

3. Set AC switches to $\phi$. (If AC switches were 5, page numbers would be printed as $5-1,5-2$, etc.

4. Press CONTINUE

\section{CARD INPUT/OUTPUT}

BLOCK 115 - LIST DECK uses the KSR 35 Teletype to list the information, one card per line.

Two or more spaces on the card are converted to a tab before output. The last block of spaces on the card is ignored. A carriage return and a line feed are typed or punched after each card. If a character cannot be found in the conversion tables, a backward slash is put in its place.

Load the deck in the card reader hopper with row 9 nearest slot, face down. Press START on the reader after the program is in core and running. When all cards have been read, another deck may be loaded. Press START on the reader to begin output.

Program may be restarted at location 100 .

BLOCK 120 - PUNCH DECK uses the paper tape punch to provide the user with an ASCII tape.

Two or more spaces on the card are converted to a tab before output. The last block of spaces on the card is ignored. A carriage return and a line feed are typed or punched after each card. If a character cannot be found in the conversion tables, a backward slash is put in its place.

Load the deck in the card reader hopper with row 9 nearest slot, face down. Press start on the reader after the progran is in core and running. When all cards have been read, another deck may be loaded. Press start on the reader to begin output.

Program may be restarted at location 100 .

\section{MEMORY DUMPS}

BLOCK 21. - OCTAL MEMORY DUMP PRINTER

1. Call printer into memory thru the DECtape reader

2. The memory block used by the printer program will be The memory block used by
typed out on the Teletype

3. To use: Enter first location to be printed on ACS

4. Press CONTINUE

5. Enter LAST location on ACS

6. Press CONTINUE

7. To REPEAT go to step 3

. Printing may be suppressed at any time by putting $\mathrm{AC}-0$ up

TO PRINT ANOTHER BLOCK GO TO STEP 3

BLOCK 22 - ABSOLUTTE MEMORY DUMP PRINTER

1. Read in program from mag tape through DECtape reader

2. Set low address in $A C$ switches

3. Set low address

4. Set high address in AC switches

5. Press CONTINUE

\section{ANALOG SETUP AND CHECK OUT}

BLOCK 60 - READ POTS

1. Read in program through DECTAPE READER. Pots are listed autonatically without further operator intervention. POTED on Block 300 could also be used for this function.

BLOCK 125 - PUNCH STEADY STATE CHECK TAPE

When a steady state has been reached by'the problem either in IC or COMP modes, the Punch Steady State Check Tape in IC or COMP modes, the Punch Steady State Check Tape automatically places the 2133 in HOLD mode, scans A, F, L, automatically places the 2133 in HOLD mode, scans A, F, L,
$M$ and $R$ points and punches the tape. Notation should be $M$ and $R$ points and punches the tape. Notation should be
made on the tape to indicate which mode the 2133 was in whade on the thape to $\frac{\text { to }}{\text { the }}$ program $\frac{\text { stic }}{\text { started. }}$

BLOCK 126 - STEADY STATE CHECK

1. The Steady State Check program is called from block 126 of the DSD Master. The program will halt in location 16001. If a voltage deviation 1init other than 0.5 volts is desired, the negative BCD value should be entered in the location whose address is in the accumulator at the time of the halt.

2. Load the paper tape in the reader.

3. When the 2133 has reached steady state IN THE SAME MODE AS WHEN THE TAPE WAS MADE, press continue, or, if the user elected to change the deviation limits, "start" at location 16002 . The program will indicate "TEST COMPLETED" when finished. 
SIMPL-1 SYSTEMS PROGRAM OPERATING INSTRUCTIONS (contd)

\section{EDITOR OPERATING INSTRUCTIONS*}

\section{INPUT - TAPE OPERATIONS}

Read one page of text

Read n lines of tape

Skip one page of text

Skip n pages of text

\section{$\underline{\text { LIST }}$}

Back up and print

Get next location tag after line $\underline{n}$

Print line $\underline{n}$

Print lines $\underline{n}$ through $\underline{m}$

Print uncommented the entire buffer

Print line $\mathrm{n}$ uncommented

Print lines $\underline{n}$ through $\underline{m}$ uncommented

Write the entire buffer

Write $\underline{n}$ pages. Equivalent to $\mathrm{K}, \mathrm{n}(\mathrm{R}, \mathrm{W}, \mathrm{K})$.

Rubout typed immediately after carriage return will

cause 1 isting of the next line. Otherwise, "rub-

out" will erase last character typed.

"Line Feed"

Print next line (if first character typed in command mode.) Otherwise, delete all typed input on this line when in append mode.

\section{PROGRAM MODIFICATIONS IN TEXT MODE}

Append

Change line $\underline{n}$

Change lines $n$ through $m$

Insert text before line $n$

Erase last character. (When rubout is first

character after a carriage return, the editor

mode is changed from text to command mode.)

\section{PROGRAM MODIFICATIONS IN COMMAND MODE}

Delete line $\underline{\mathrm{n}}$

Delete lines $\underline{\mathrm{n}}$ through $\mathrm{m}$

Kill the text buffer

External insert. Insert $n$ lines of text from paper tape after line $\mathrm{m}$

Individual character correction on line $n$. (First

"rubout" stops printing. Remaining rubouts erases characters one at a time.)

Print first line (if first character typed). Otherwise delete all typed input on this line.

\section{OUTPUT - TAPE OPERATIONS}

Punch n lines of tape feed

Punch and next page. Equivalent to $P, S, K, R$

Punch, duplicate, and read. Equivalent to $P, S, m-1(T), R$

Punch and kill. Equivalent to $P, S, K$

Punch the contents of the buffer

Punch line $\underline{n}$

Punch lines $\underline{\text { n }}$ through $\mathrm{m}$

Punch form feed (FIODEC:stop code)

Duplicate $n$ pages of tape. Equivalent to $K, n(R, P, S, K)$

\section{SPECIAL FUNCTIONS}

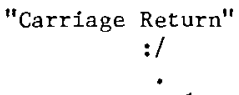

Complete specified action

Total line indicator

Current address indicator

Down - produces normal operation

Up - stops printing or punching

*If the D.T. (HIGH) READER is in core the editor is called from mag tape by:

1. Setting tape unit number in $A C S w .0-5$

2. Setting block number (26) in AC Sw. 5-17

3. Setting address switches to 17640 or 7640

4. Pressing START

5. To restart editor without destroying contents of buffer address 22 and press START 
SIMPL-1 SYSTEMS PROGRAM OPERATING INSTRUCTIONS (contd)

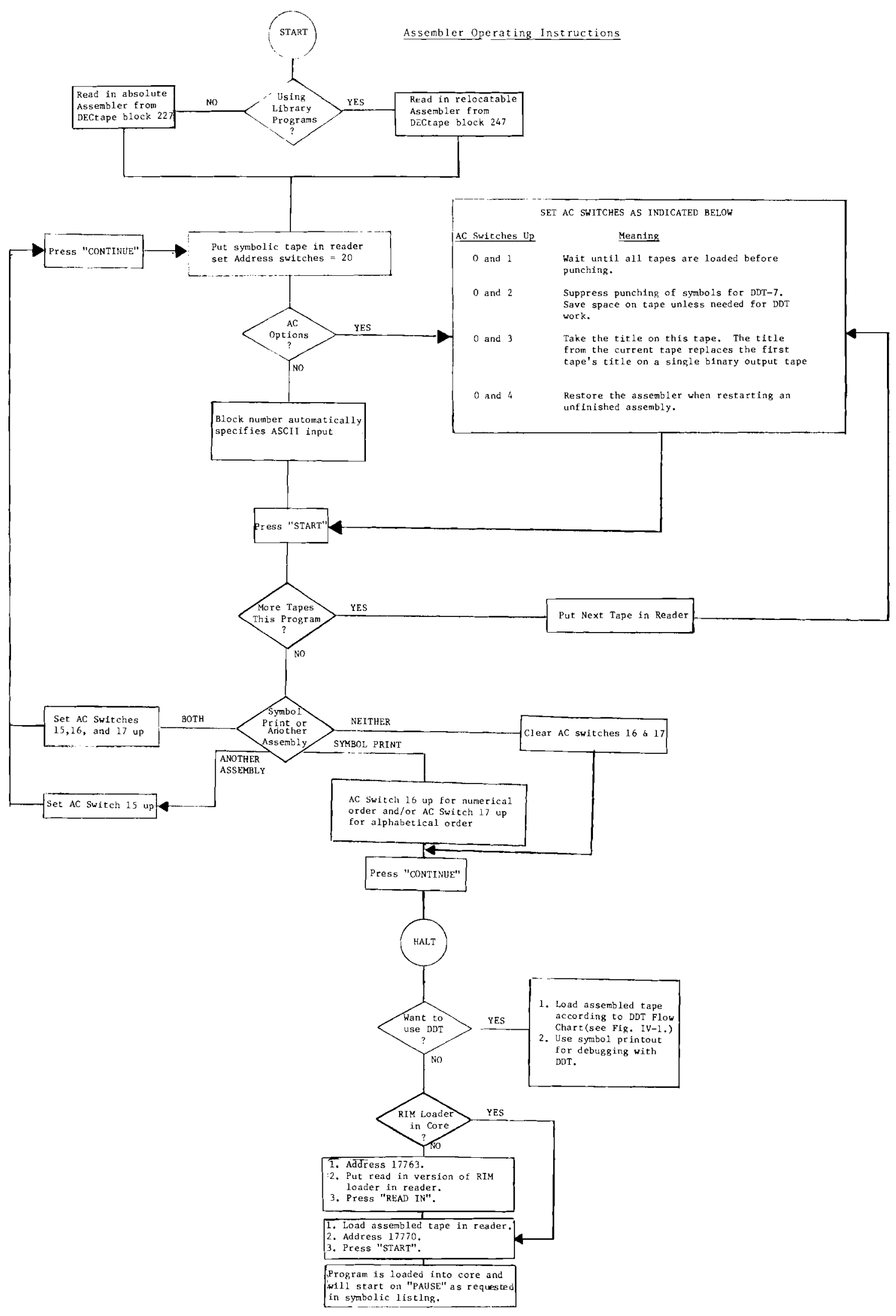


SIMPL-1 SYSTEMS PROGRAM OPERATING INSTRUCTIONS (contd)

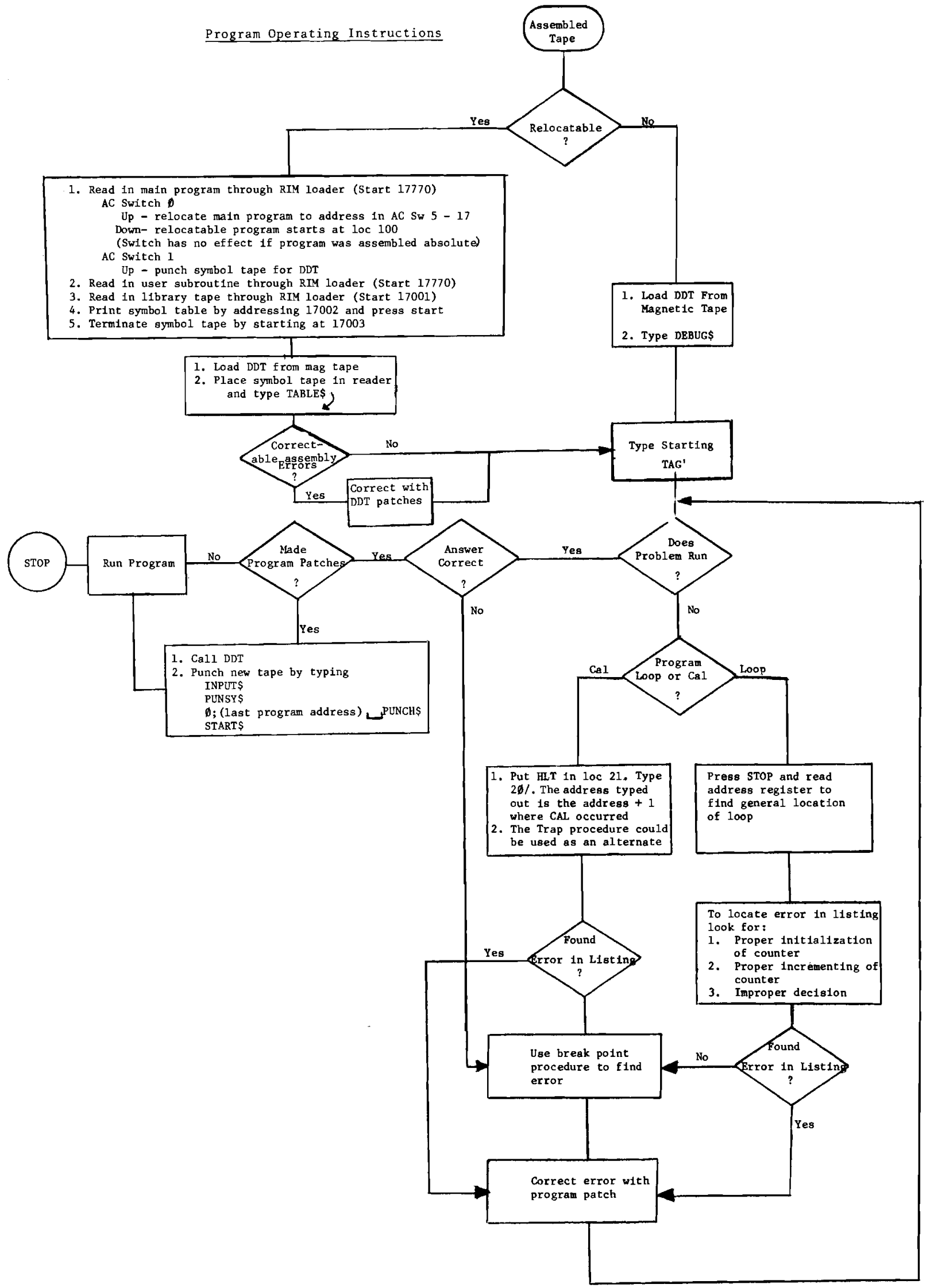




\section{SYMBOL AND FORMAT ERRORS}

The appearance of the diagnostic is:

ERROR

OCTAL ADDRESS

SYMBOLIC ADDRESS

The error legend is:

\begin{tabular}{|c|c|}
\hline Error & $\underline{\text { Meaning }}$ \\
\hline IFC & $\begin{array}{l}\text { Illegal format in symbolic address tag. The tag } \\
\text { is ignored, (See page } 17 . \text { ) } \star \star\end{array}$ \\
\hline IF I & $\begin{array}{l}\text { An expression using CHAR or FLEX was formed } \\
\text { improperly. (See page } 38 \text {.) }\end{array}$ \\
\hline IFL & Illegal format in a PUNDEF list. (See page 26.) \\
\hline IFP & $\begin{array}{l}\text { Illegal format in a parameter assignment. The } \\
\text { assignment is ignored. (See page 8.) }\end{array}$ \\
\hline IFS & $\begin{array}{l}\text { START or PAUSE used incorrectly. Assembly con- } \\
\text { tinues as if START or PAUSE had been used with } \\
\text { no expression following. (See page 17.) }\end{array}$ \\
\hline ILF & $\begin{array}{l}\text { Illegal format in a paeudo-instruction such as BAR. } \\
\text { The pseudo-instruction is ignored. }\end{array}$ \\
\hline INS & $\begin{array}{l}\text { An illegal format in a PUNDEF list } \rightarrow \text { two commas } \\
\text { appeared in a row or a digit appeared. }\end{array}$ \\
\hline LIT & $\begin{array}{l}\text { An illegal character was found in a PUNDEF list. } \\
\text { The character is taken as a terminator. }\end{array}$ \\
\hline MDT & $\begin{array}{l}\text { The value of the complex symbolic address assign- } \\
\text { ment (tag) and the location counter disagree. The } \\
\text { symbolic address tag is redefined if possible. } \\
\text { (See page } 17 . \text { ) }\end{array}$ \\
\hline SCE & $\begin{array}{l}\text { Storage capacity of the symbol table was exceeded. } \\
\text { No recovery is possible. }\end{array}$ \\
\hline TUA & $\begin{array}{l}\text { Too many undefined symbols appeared in a symbolic } \\
\text { address assignment (tag). Location counter remains } \\
\text { unchanged. (See page 17.) }\end{array}$ \\
\hline UBR & $\begin{array}{l}\text { An undefined symbol appeared in a BAR pseudo-instruc- } \\
\text { tion. The setting of BAR remains unchanged. }\end{array}$ \\
\hline
\end{tabular}

\section{SYMBOL DEFINATION ERRORS}

The appearance of the diagnostic is:
ERROR
PREV IOUS VALUE
SYMBOL
NEW VALUE

The error legend is:

Error
MDT
RDA $\begin{aligned} & \text { A previniously defined symbol was redefined with a conma. } \\ & \text { (See page 17.) }\end{aligned}$
An attempt was made to redefine a permanent symbol with
a comma. The symbol was not redefined.
A permanent symbol was redefined. (See page 8.)
CHARACTER AND SYMBOL ERRORS

The appearance of the diagnostic is:

$$
\text { ERROR OCTAL ADDRESS SYMBOLIC ADDRESS CAUSE }
$$

The error legend is:
Error

ICH

ILP

UPA

UPN

UST
A character not part of the Assembler's source language was used. The character is ignored. (See page 7.)

A character read from tape did not have an odd number of holes across the line. Place the correct character (if possible) in bits 12-i7 of the ACS and press CONTINUE.

A An undefined symbol appeared in an absolute address assignment $(/)$. The current address indicator remains unchanged.

An undefined symbol appeared in a parameter assignment. The assignment is ignored. (See page 8.)

An undefined symbol appeared in a PUNCH pseudo-instruction. The symbol is ignored.

Ar undefined symbol appeared in a START or PAUSE instruction. The symbol is ignored and the START or PAUSE taken alone. (See page 17.)

*With the exception of SCE (Storage capacity exceeded) and ILP (11legal parity),

assembly continues automatically after error message has been printed.

$\star \star$ Page references refer to the PDP -7 Symbolic Assembler Programming Manual (DIGITAL-7-3-S) 


\section{ABSOLUTE VERSUS RELOCATABLE ASSEMBLY}

The advantages for using a relocatable assembler are:

1. Subroutines (both user and library) need only be assembled once.

2. Main programs may be modified and changed without destroying the 1 inkage between the main program and previously written subprograms.

3. Core memory is more effectively utilized because only the ones needed are $10 a d e d$ into memory.

4. Unused portions of core can be eliminated by program packing.

A relocatable assembly is specified if the first non-comment program entry is not an absolute address assignment. An absolute assembly is specified if the first non-comment program entry contains an absolute address assignment (i.e. 642/). For example in the 1 isting

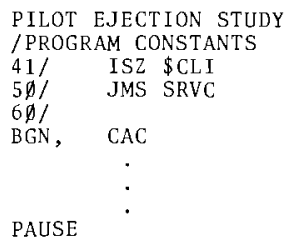

would be assembled absolute because the first non-comment entry (41/ ISZ \$CLI) contains an absolute address assignment (41/). The starting address BGN is stored in 1ocation 60 . [The 10cations between 40 and 57 are reserved at API channels and are not generally used for other purposes (see item 10 of Appendix B).]

The following listing would be assembled relocatable:

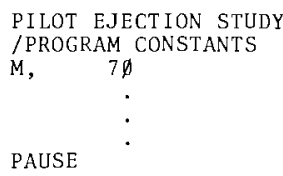

As indicated in the program operating instruction of Appendix D-8, the program would be loaded at memory location 100 if AC switch $\emptyset$ is down, or the starting location is taken from AC switches 5-17 if AC switch $\emptyset$ is up.

The following technique is recommended for referencing absolute addresses when using the relocatable assembler:

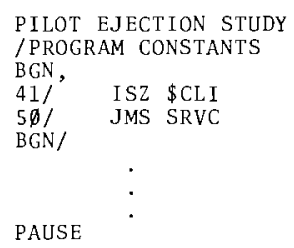

Absolute memory locations can also be placed between the end of a main program and prior to beginning an API clock service routine. For example.

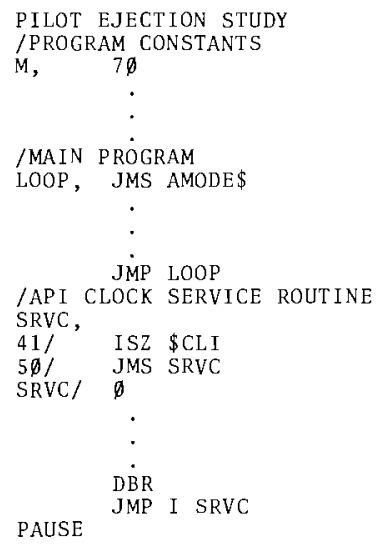

Programing errors relating to absolute or relocatable assembly can be avoided by noting that programs starting relocatable must end relocatable. Conversely, programs beginning absolute must end absolute. 
SIMPL-1 SYSTEMS PROGRAM OPERATING INSTRUCTIONS (contd) DDT OPERATING INSTRUCTIONS

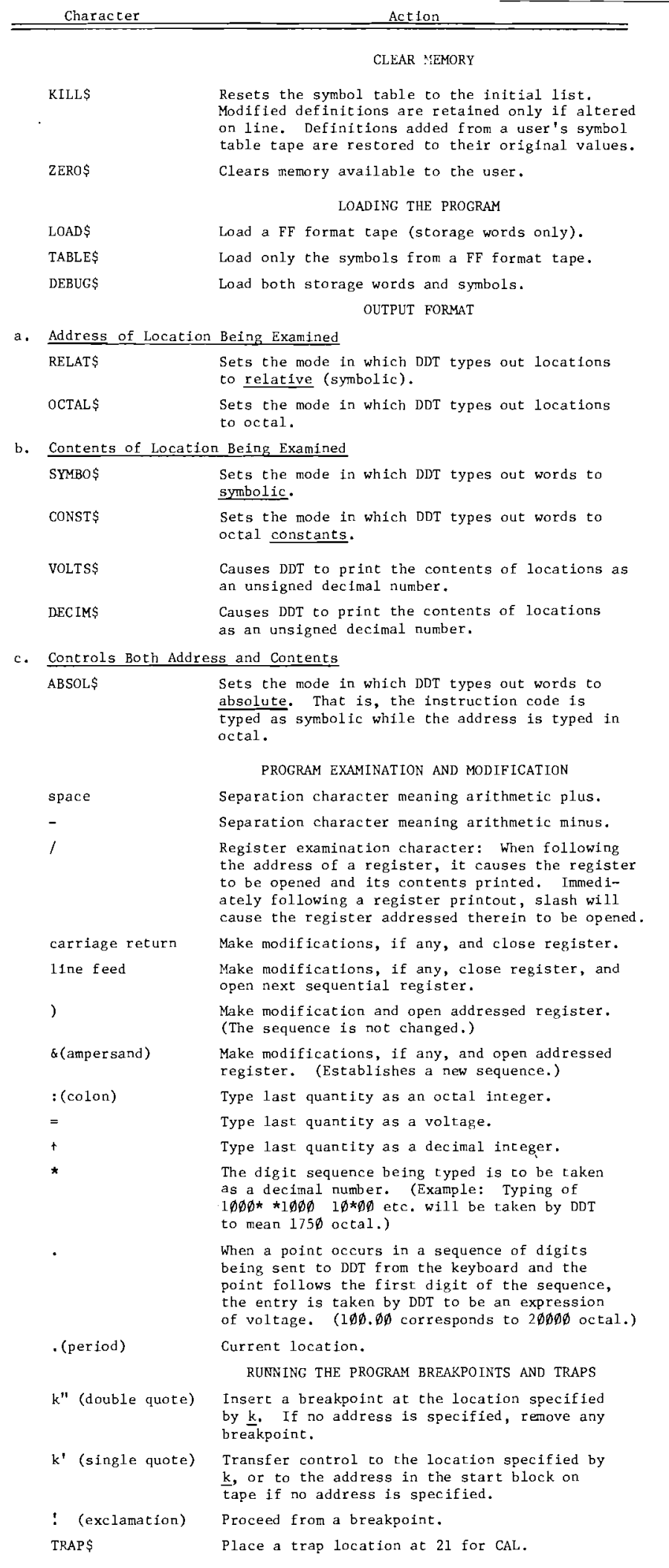

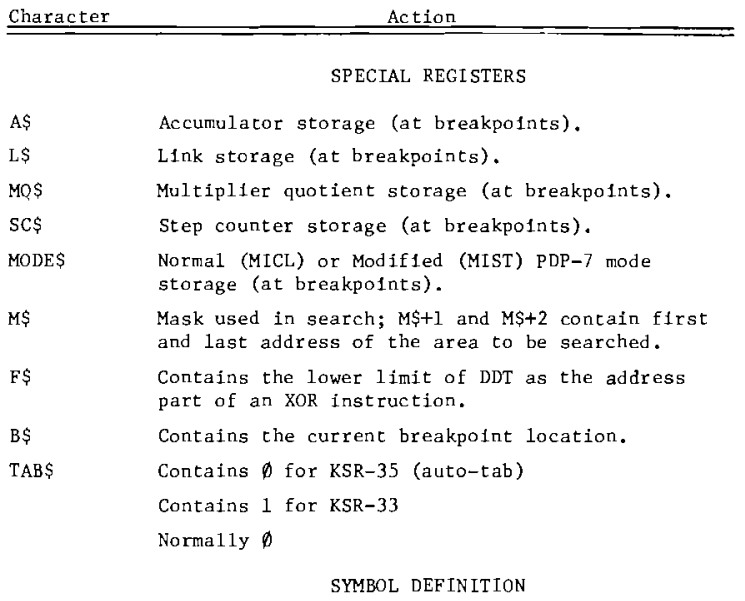

$k$, Define the symbol $\underline{k}$ as the tag of the currently (...) Define the enclosed symbol as the value preceding the $($.

SEARCHING

N WORDS Search for all occurrences masked with M\$ of Search for all occut
the expression $\mathrm{N}$.

$N$ NOT\$ Search for all words not equal to the expression $N$ after masking with MS.

N ADDRE\$ Search for all words masked with MS with the same effective address as $\mathrm{N}$.

\section{MISCELLANEOUS OPERATIONS}

k/f Execute the expression $\underline{k}$ as an instruction.

QS Last quantity typed out by DDT.

BAUDOS Causes DDT to read the symbols on a funny format Causes DDT to read the symbols on a funny for
paper tape (assembler output, DDT output, or paper tape (assembler output, DDT output, or
linking loader output) as 6 bit baudot trim codes. linking loader output) as 6 bit baudot trim codes.
This instruction should be used for all programs This instruction should be used for all programs
assembled prior to February 1,1968 or for any other programs not assembled on the SIMPL1 Hybrid Assembler.

ASCIIS Causes DDT to read the symbols on a funny format paper tape (assembler output, DDT output, or 11 nk 1 ng paper tape (assembler output, DDT output, or linking
loader output) as 6 bit ASCII trim codes. This will loader output) as 6 bit ASCII trim codes. This will be the normal operating mode of DDT and should only
be necessary after a tape of the type described in be necessary after a tape of the type described
BAUDOS has been loaded. The ASCII symbol mode BAUDOS has been loaded. The ASCII symbol mode
applies to all tapes assembled on the BNW - SAS applies to all tapes assembled on the BNW - SAS Hybrid DDT output tapes, and linking loader symbol punch tapes.

PUNCHING OPERATIONS

INPUTS Punch the input block.

N PUNCHȘ Punch the contents of $N$.

N; M Punchș Punch $N$ to $M$, inclusive.

PUNSYş Causes DDT to punch on paper tape the entire nonpermanent symbol cable.

START\$ Punch a start block. SPEGIAL CHARACTERS

"RUBOUT" Deletes last typing sequence. For example, suppose the instruction in location JOE is JMS HMPY $2 \$$ and it's desired to modify the instruction to JMP STRT. it's desired to modify th However, in the process of making the modification an error was made (i.e., an $S$ was typed for a $P$.) The Teletype output would appear as shown below (underlined expressions are typed by user and
expressions not underlined are type by DDT.)

$\begin{array}{lll}\frac{\text { JOE} /}{\check{I}} & \text { JMS HMPY2S } & \frac{\text { JMS }}{\uparrow} \text { ?JMP STRT } \\ \text { JMS STRT } & \text { "RUBOUT" }\end{array}$

If the D.T. (HIGH) READER is in core, DDT

2. Setting block number (44) in AC SW 5-17

2. Setting block number (44) in AC SW $5-17$
3. Setting address switches to 17640 or 7640

3. Setting addres

5. To restart DDT address 16000 and press starc 
APPENDIX H

\section{TYPICAL PROGRAMMING AND OPERATING ERRORS}

\section{PROGRAMMING ERRORS}

1. Are dollar signs (\$) used on all library functions?

2. Are al1 tags correctly spelled? (i.e., have you said TMP when it should be TEMP?)

3. Make sure spaces are not substituted for tabs. If bugs in program cannot be found check to see if spaces were substituted for a tab by re-listing the source paper tape program.

4. Do JMP instructions contain.$+ N$ rather than . $+\mathrm{N}$ or . $\mathrm{N}$ ?

5. Have you used the correct calling sequence for library subroutines?

6. Have you confused decimal, octal, and voltage numbering systems?

7. Have you used literals properly? i.e., LAC 36 means load the accumulator with contents of location 36. In contrast LAC ( 36 means load the accumulator with the octa1 number 36 .

8. Have you used slashes for comments?

9. Have you confused absolute and relocatable assembler addresses identification?

10. When making DDT decimal integer patches use * rather than ' as the decimal indicator. (In DDT the use of (') starts the program running.)

11. Have pseudo-instruction names been used as tags?

\section{OPERATING ERRORS}

1. ADC Channe1s show overload and 1imit. DAC Channe1s do not show overload. Be sure to check ranges of variables trunked to analog to insure that most significant bits are not lost.

2. Is the write lock switch on the DECtapes in the center position?

3. Are tape unit switches addressed to the right units?

4. Have assembled tapes been loaded in the proper manner? (Remember assembler output tapes are loaded upside down and backwards.)

5. Have you used memory locations 40-57? If so examine API indicator lights to see if spurious API pulses have clobbered your program. 
APPENOIX I

NUMBERING SYSTEMS ANO THEIR APPLICATIONS

OCTAL

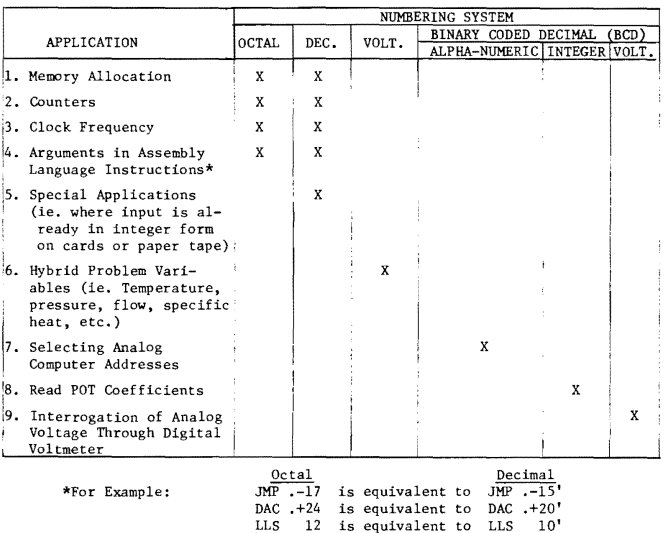

BINARY

Every PDP-7 digital computer operation is performed in the binary numbering system. Other numbering systems (such as BCD) are needed for equipment compat selector system must be coded in alpha-decimal to $B C D$. However, all operatio
performed by the digital computer utilizes the binary numbering system.

The binary numbering system only consists of two numbers ( 1 and 1$)$. The value of a binary number is a f
number 11091 may be written as:

$$
\begin{aligned}
11 \emptyset \emptyset 1_{2} & =1 \times(2)^{4}+1 \times(2)^{3}+0 \times(2)^{2}+0 \times(2)^{1}+1 \times(2)^{0} \\
& =16+8+0+0+1 \\
& =2510
\end{aligned}
$$

The figure below gives a formalized conversion method along with the bit value of a standar

BIT
POSition

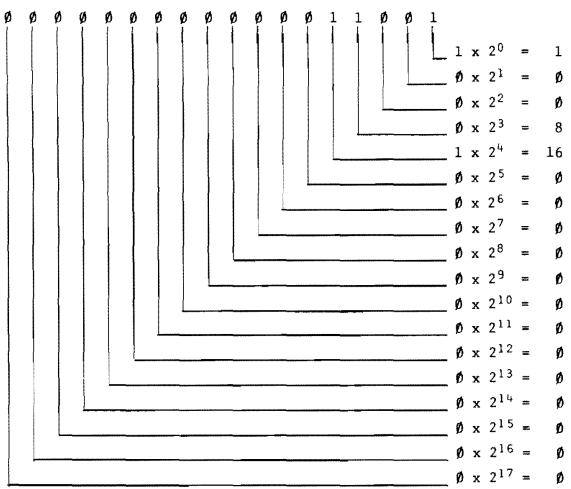

Decimal Equivalent $=25$

The procedure of converting from decimal to binary is:

REMAINDER

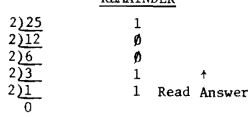

Even though the digital computer performs all operations in binary arithmetic, it is sometimes more convenient to convert a binary number through an octal number to obtain the decimal equivalent. The conversion from binary to octal can be done by segenting the binary anber in groups of thre before making the visual conversion. Three binary places can be used to represent any number between 0 and 7 as shown below:

$$
\begin{aligned}
& \begin{array}{ccc} 
& \text { OCTAL } & \text { DECIMA } \\
000 & 0 & 0 \\
001 & 1 & 1 \\
010 & 2 & 2 \\
011 & 3 & 3 \\
100 & 4 & 4 \\
101 & 5 & 5 \\
110 & 6 & 6 \\
111 & 7 & 7
\end{array} \\
& \text { The binary number } 11001 \text { can be grouped as: } \\
& \text { D11 } \begin{aligned}
\text { Di } & \text { = Binary Number } \\
3 \quad 1 & =\text { Octal Number }
\end{aligned} \\
& \text { or } 011011_{2}=31_{8}
\end{aligned}
$$

The procedure for converting from octal to decimal is:

$$
\begin{aligned}
31_{8} & =3 \times(8)^{1}+1 \times(8)^{\circ} \\
& =24+1 \\
& =2510
\end{aligned}
$$

To convert from decimal to octal the following procedure is used.

$$
\begin{aligned}
& \begin{array}{l}
\frac{8) 25}{8 \frac{3}{0}} \\
\quad \frac{1}{3}{ }^{\text {REMAINDER }} \\
\text { Read Answer }
\end{array} \\
& \text { or } \quad 2_{10}=31_{8}
\end{aligned}
$$

\section{DECIMAL (INTEGERT)}

The decimal numbering system is understood by almost everyone. (For example: $25_{10}=2 \times(10)^{1}+5 \times(10)^{0}$ ) However, extreme care must be exercised in using SIMPLl software to avoid confusion caused by the integer decimal system.

Decima1 integers should not be used to represent problem variables such as temperature, pressure, specific heat, concentration, etc. The hybrid subroutines have been conditioned to recognize problem variables as "digital voltages."

Decimal integers may be used for those applications specified in the RECOMMENDED NLMBERING, SYSTEM APPLICATIONS.

\section{BINARY VOLTAGES}

The term binary voltage (or just voltage) is used to denote the special numbering system used by SIMPL1 arithmetic routines. The equation relating the decimal equivalent $(\mathrm{N})$ to a "binary voltage" (v) is:

$$
N=\frac{\mathrm{V} * 8192}{100}
$$

In essence, the eighteen bit digital word is conditioned to recognize algebraic sign and seventeen bits of numerical information. The smallest representable voltage is +0.012 and the largest is \pm 1599.99 . In other words, the resolution is 0.012 volts.

The reason for adopting a new numbering system is the necessity for relating analog voltages to digital integers. The largest decimal number representable on an analog computer is 100.0 volts,

\begin{tabular}{|c|c|}
\hline ALPHA & DECIMAL \\
\hline A & 0 \\
\hline $\mathrm{F}$ & 1 \\
\hline G & 2 \\
\hline $\mathrm{k}$ & 3 \\
\hline L & 4 \\
\hline M & 5 \\
\hline $\mathrm{P}$ & 6 \\
\hline $\mathrm{R}$ & 7 \\
\hline T & 8 \\
\hline $\mathrm{U}$ & 9 \\
\hline
\end{tabular}
and the accuracy of most analog computing elements is .01 volts. on the other hand, the largest seventeen bit decimal integer is $2^{18}-1$ or 262,143 . The smallest integer is 1 (decimal fractions are not allowed in the integer decimal system.) To make the numbering systems of both machines compatible, the integer representation of the digital's seventeen bit word was rescaled to cover the range of \pm 1599.99 to \pm .012. However, numerical transfer to and from each computer must be less than \pm 99.99 .
ALPHA-DECIMAL TO BINARY CODED DECIMAL(BCD)

The analog compurer address select system is coded for a special alpha-decimal to BCD equivalence as shown in Table I.

TABLE I. Alpha-Decimal to BCD Conversion

The format and an example for addressing POT 126 is shown in Table II.

TABLE II. Accumulator values for $8421 \mathrm{BCD}$ Used for Analog

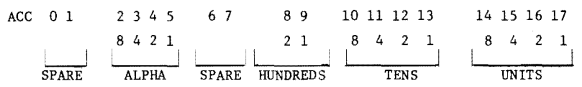
ADDRESSING EXAMPLE

$\begin{array}{cccccl}\mathrm{XX} 0110 & \mathrm{XX} & 01 & 0010 & 0110 & \text { BCD ADDRESS } \\ \mathrm{P} & & 1 & 2 & 6 & \text { ALPHA-DECIML ADDRESS }\end{array}$ BCD INTES,ER

The integer binary coded decimal numbering system must be used for reading POT coefficients from the analog computer or setting POT from the digital computer.

The BCD integer format is show in Table III.

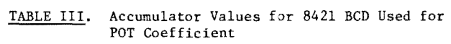

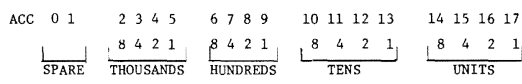

$\widehat{\text { SPARE }} \overline{\text { THOUSANDS }}$ HUNDREDS

The integer BCD to binary conversion subroutine (IDBCS) and integer binary to $\mathrm{BCD}$ subroutine ( $\mathrm{BDC}$ ) are included in the hybrid software to perform the necessary PoT coefficient conversions.

\section{BCD VOLTAGE}

The digital output of the analog computers digital voltmeter is coded according to the format shown in Table IV.

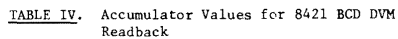

$$
\begin{aligned}
& \text { ACC } \quad 0 \quad 1 \quad 2345 \quad 6789 \quad 10111213 \quad 14151617
\end{aligned}
$$

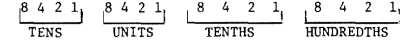

$$
\begin{aligned}
& \text { OR HUNDREDS TENS UNITS TENTHS } \\
& \begin{array}{l}
\text { OR HUNDREDS } \\
\text { DECIMAL (=XXX. } x \text { THENS ONE }
\end{array} \\
& - \text { SIGN } \begin{array}{r}
(\text { MINUS }=\text { ONE) } \\
\text { (PUUS }=\text { ZERO) }
\end{array}
\end{aligned}
$$

The digital voltmeter can be used to interrogate any analog voltage without using a separate ADC channel. When this is done the analog BCD voltage presented to the digital computer's accumulator must be converted to a digital "binary voltage" before it can be used for a computation. This conversion is performed by the voltage $B C D$ to voltage binary (VDBCS) conversion subroutine. 
APPEMDIX J
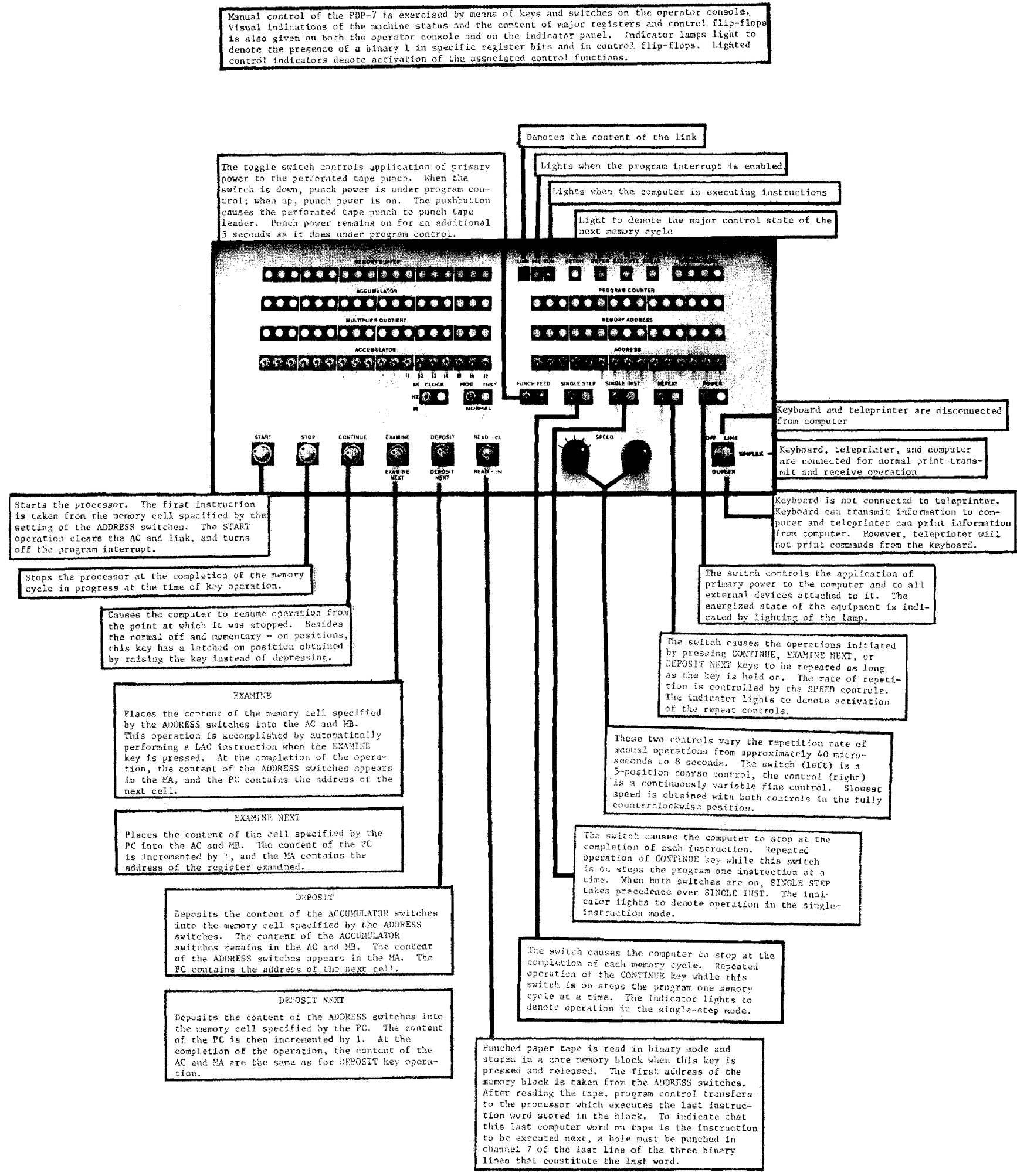

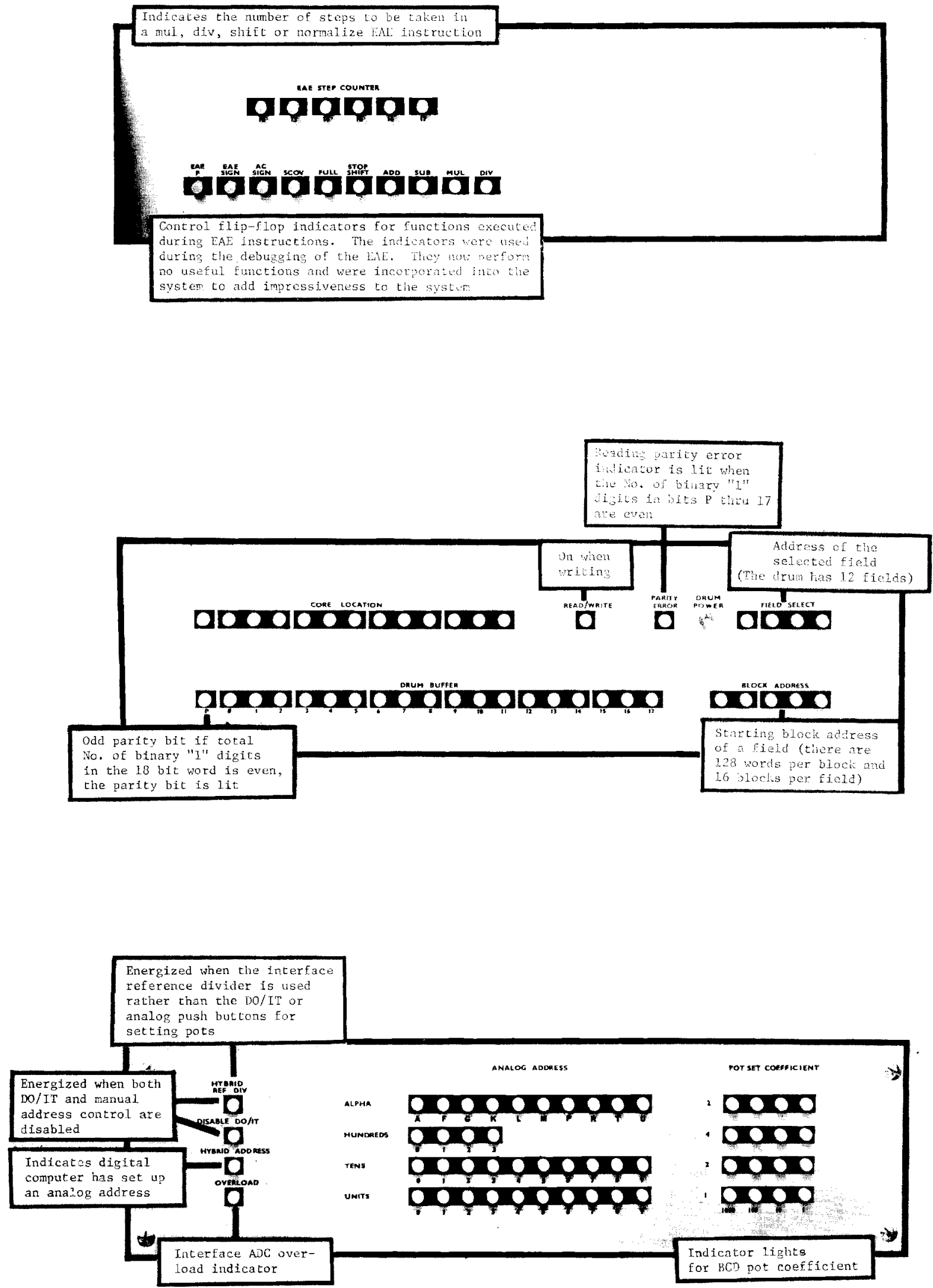

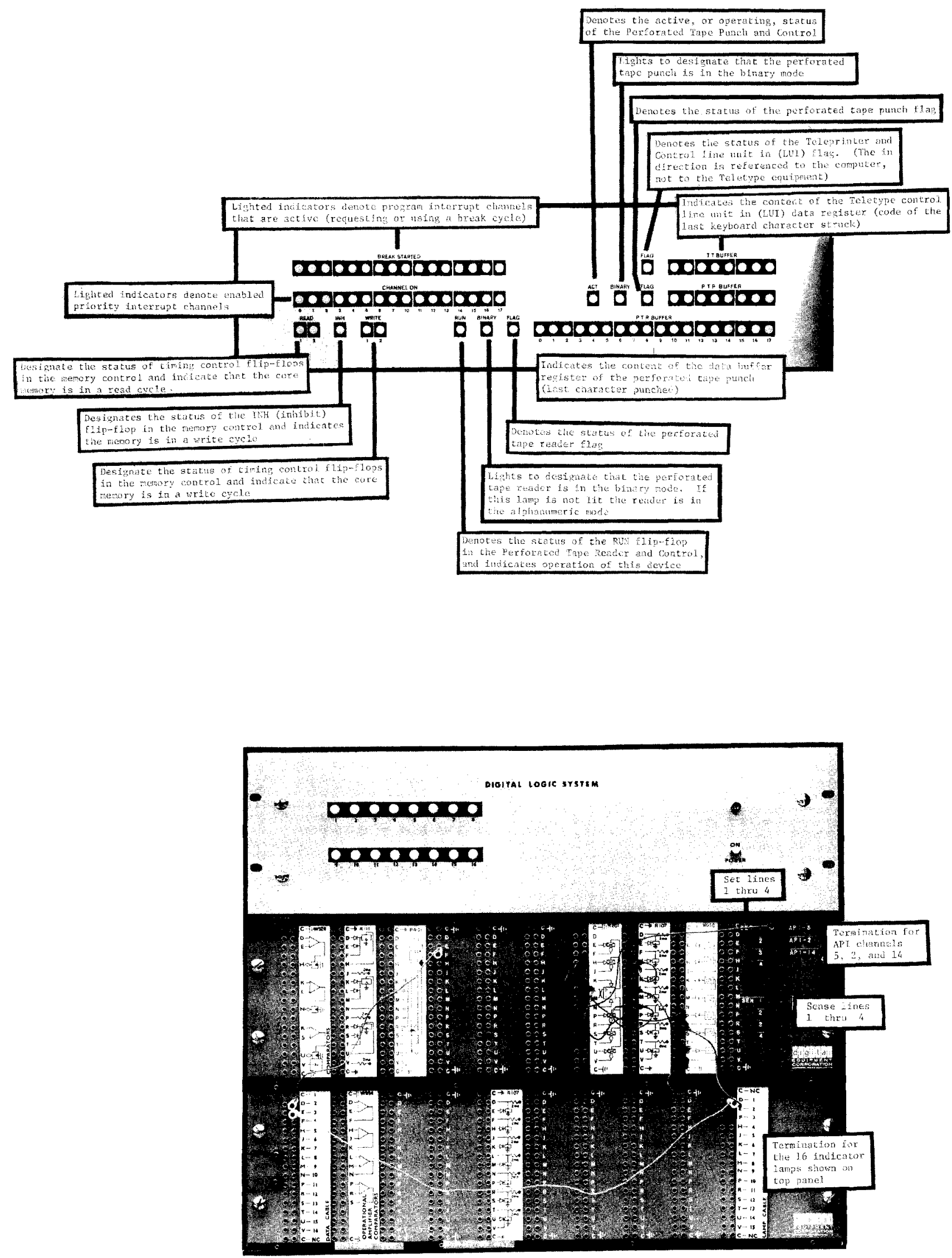


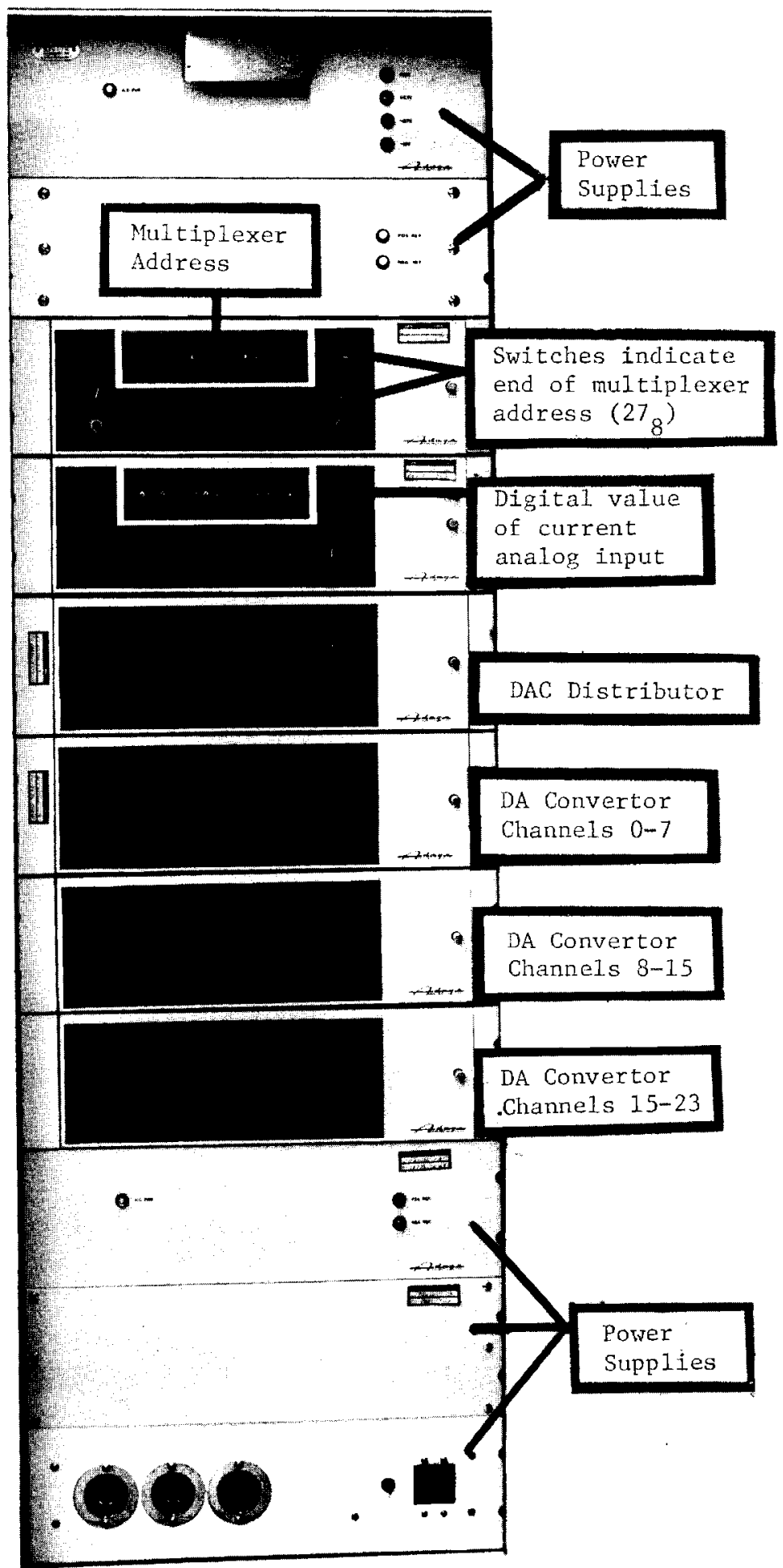


APPENDIX K

EXAMPLE MULTIPLY AND DIVIDE PROGRAMS WITHOUT USING SIMPL-I FUNCTIONS

To use the multiply (both signed and unsigned) instructions, the basic calling sequence is to load the accumulator with one number before the instruction and to have the other number in the location following the multiply instruction. An example follows:

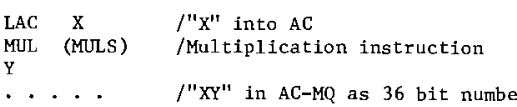

.... $/$ "XY" in $\mathrm{AC}-\mathrm{MQ}$ as 36 bit number

In both the signed and the unsigned multiplication " $\mathrm{Y}$ " (see example above) must be the absolute value of the number to be multiplied. The sign of "Y" should be in the link; therefore, if " " is a constant the link can be set or cleared preceeding the multiplication to indicate the proper sign of "Y". With this type of multiplication " $\mathrm{Y}$ " can be of

CLL (STL) $\begin{aligned} & \text { /CLL if "Y" is positive; STL if "Y" is } \\ & \text { /negative }\end{aligned}$
LAC X
MUL (MULS)
Y
/. . .

any magnitude up to $777777_{8}$ and still be considered positive.

If " " is a variable it can be loaded into the location

following the multiply instruction and its sign can be placed into the link with the following instructions:

$\begin{array}{lll}\text { LAC } & \mathrm{Y} & \begin{array}{l}\text { /Positive or negative variable } \\ \text { /SSM }\end{array} \\ \text { DAC } & .+3 & \\ \text { LAC } & \mathrm{X} & \\ \text { MUL } & \text { (MULS) } & \\ \emptyset & & \\ . . & \text {. . } & \text { /XY in AC-MQ as } 36 \text { bit number }\end{array}$

The instruction GSM places the sign bit of the variable into the link and takes the absolute value of the number. Once again the link could be set or cleared in place of the GSM instruction and " $Y$ " would be considered as an 18 bit number.

When using the MUL instruction the variable " " in the examples above will be considered as an 18 bit positive number. If the instruction MUS is used, " $\mathrm{X}$ " will be a 17 bit number with bit $\emptyset$ as its sign. The multiplication will take this sign and the value of the link into consideration to produce the correct sign on the product.

Al1 of the examples above are valid where " $\mathrm{X}$ " and " $\mathrm{Y}$ " are both octal integers or where one of them is an octal integer and the other a scaled number such as a voltage. When multiplying two scaled numbers together it is necessary to take the scaling into account because the multiplication effectively squares the scale factor. Because of this, it is necessary to divide the product by the scale factor to provide proper scaling. When voltages are being used this scale factor is $2^{13} / 100$.

Most generally, when one is multiplying two voltages, it is convenient to perform the multiplication in an analog fashton where the product would be divided by 100 . Therefore, the analog type multiplication of two voltages is easily performed by dividing the product by $100\left(2^{13} / 100\right)$ which is merely $2^{13}$. Since the product is normally found in the $\mathrm{AC}-\mathrm{MQ}$ as a 36 bit number it is necessary to divide by $2^{13}$ and then multiply by $2^{18}$ to get the answer into the AC. This can be accomplished by merely multiplying by $2^{5}$. An example of this follows:

$\begin{array}{ll}\text { LAC } & \text { Y } \\ \text { GSM } & \\ \text { DAC } & +3 \\ \text { LAC } & \mathrm{X} \\ \text { MULS } & \\ 0 & \\ \text { LLS } & 5 \\ \text {. . . . }\end{array}$

/Shift left 5 bits - (multiply by $2^{5}$ ) $/ \mathrm{XY} / 100$ in $\mathrm{AC}$
The answer, XY/100, can not be larger than 1599.99 volts for this type of multiplication.

If increased speed is desired in a multiplication and it is known that the variable " $\mathrm{X}$ " is less than an 18 bit number, the multiply instruction can be modified slightly. Instead of using MULS once can use MULS- $C$ where $C$ is the number of bits difference between 18 and those contained in the number " $\mathrm{X}$ ". To multiply by a fifteen bit number once could use MrLS- 03 since $18-15=3$. The reason this is possible is that multiplication is performed through a shift-add sequence; if a bit is zero no addition is necessary. It must be recognized, therefore, that the result in this type of a multiplication is actually the product times $2^{\mathrm{C}}$ in the AC-MQ. An example follows:

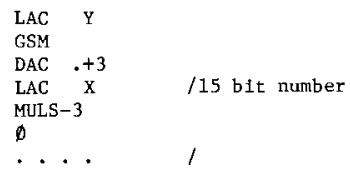

Advantage can be taken of this property of multiplication in multiplying two voltages. In a previous examp1e, the result in the $A C-M Q$ was shifted five places right to give the properly scaled answer in the AC; the instructions could be changed as follows:

$\begin{array}{ll}\text { LAC } \quad \text { Y } & \text { /Voltage variable } \\ \text { GSM } & \\ \text { DAC } .+3 & \text { /Voltage variable } \\ \text { LAC X } & \\ \text { MULS-1 } & \\ \text { LLS } 4 & / \text { XY/100 in AC } \\ . . . & \end{array}$

The MULS instruction always places bit of the accumulator into the EAE sign bit register and then takes the absolute value of the number which always makes bit a zero. Hence, as shown in the above example, it is possible to ignore this bit in the multiplication and then reduce the shift count in the next instruction. This saves approximately 0.2 micro-seconds for each multiplication.

The HMPYIS and HMPY2\$ subroutines for the multiplication of two voltages use the type of sequence shown in the above example. For a general multiplication this is the fastest sequence presently available. More speed could be obtained, however, by limiting the slze of the numbers. Also, these multiplication subroutines do take time for checking for overflow in the result of the multiplication and locking the result at either + or -1599.99 volts. If it is felt in a particular case that maximum speed must be obtained and it is unnecessary to check for overflow then a fast multiplication subroutine follows:

/Calling Sequence

$$
\begin{array}{lll}
\text { /LAC } & \mathrm{X} & / \mathrm{X} \text { input } \\
\text { /JMS } & \mathrm{MPY} & \\
\text { /LAC } & \mathrm{Y} & / \mathrm{Y} \text { input }
\end{array}
$$

MPY,

$$
\text { GSM }
$$$$
\mathrm{DAC} .+4
$$$$
\text { XCT I MPY }
$$

ISZ MPY

MULS-1

$\emptyset$

LLS 4

JMP I MPY

The HMPY $2 \$$ subroutine averages 35-40 micro-seconds. By sacrificing checking for overflow within the subroutine this average $t$ ime is changed to 27.5 - $33.75 \mathrm{micro-seconds,} \mathrm{or} \mathrm{a} \mathrm{savings} \mathrm{of} 6.25-7.5 \mathrm{micro-}$ seconds. In cases where overflow is a concern it could be checked at the return point of the calling sequence. 


\section{EXAMPLE MULTIPLY AND DIVIDE PROGRAMS WITHOUT USING SIMPL-1 FUNCTIONS (contd)}

\section{DIVIDE WITH DIV or DIVS}

The divide instructions cause the 36 bit number in the AC-MQ to be divided by the 18 bit number following the instruction. The quotient is in the $M Q$ and the remainder is in the AC. An example follows :

$$
\begin{array}{ll}
\text { DIV } & \text { (DIVS) } \\
\text { /Divide unsigned or signed } \\
\text { / } \\
\text { /... }
\end{array}
$$

(In many special cases it is useful to have a 36 bit number for the numerator, but for the examples shown here, this number will be limited to 18 bits.)

With either the signed or unsigned division the value " $Y$ " in the above example must be the absolute value of the variable or constant. The sign of " $\mathrm{Y}$ " should be in the link. If " $\mathrm{Y}$ " is a constant the link can be set or cleared before the divide instruction to indicate the proper sign of "Y".

$\begin{array}{lll}\text { CLL } & \text { (STL) } & \text { /CLI for positive "Y"; STL for negative " } \mathrm{Y}^{\prime} \\ \text { LAC } & \mathrm{X} & \text { /Numerator } \\ \text { LRSS } 18^{\prime} & \text { /Load in MQ } \\ \text { DIV (DIVS) } & \text { /Absolute value } \\ Y & \text { /Quotient in MQ; remainder in AC } \\ . . . & \end{array}$

If " $Y$ " is a variable its absolute value can be loaded in the register following the divide instruction and its sign can be stored in the link.

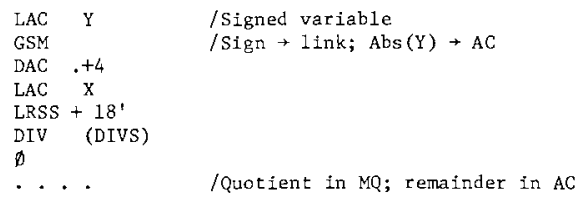

of course, the link could be set or cleared in place of the GSM instruction and " $\mathrm{Y}$ " would be considered as an 18 bit number with its sign specified in the link.

When using the unsigned divide instruction (DIV) the numerator " $\mathrm{X}$ " will be considered as an 18 bit positive number. If the instruction DIVS is used " $\mathrm{X}^{\mathrm{t}}$ will be a 17 bit number with bit as its sign. The division will take this sign and the value of the link into consideration to produce the correct sign on the quotient.

A11 of the above examples are valid where " " is either an octal integer or a scaled number, such as a voltage, as long as the denominator is only an octal integer. When dividing two scaled numbers it is necessary to take the scaling into account because the division effectively cancels the scale factor. The answer must be multiplied by the scale factor which in the case of voltages is $2^{13} / 100$.

Most generally, when one is dividing two voltages it is convenient to perform the division in an analog fashion where the answer is $100 \mathrm{X} / \mathrm{Y}$. Therefore, the analog type division of two voltages is easily performed by multiplying the quotient by $100\left(2^{13} / 100\right)$ which is merely $2^{13}$. Instead of multiplying the quotient by $2^{13}$ the same result can be accomplished by multiplying the numerator by $2^{13}$. An example of this follows:

$\begin{array}{ll}\text { LAC } \quad & \\ \text { GSM } & \\ \text { DAC }+5 & \\ \text { LAC } X & \\ \text { LRSS }+18^{\circ} & \text { /Load in MQ } \\ \text { LLSS }+13^{\prime} & \text { /Multiply by } 2^{13} \\ \text { DIV (DIVS) } & \\ \not & \end{array}$

Obviously, one could say that instead of dividing by $2^{18}$ and multiplying by $2^{13}$ it would be better to divide by $2^{5}$, thereby accomplishing the same thing with one instruction. This is valid except that it is necessary to take into accourt the final 13 bits in the MQ, making certain that they are all zeros in the case of a positive " $\mathrm{X}$ " and all ones in the case of a negative " $\mathrm{X}$ ". This is accomplished with the shift instructions in the above example because the sign bit is carried into the lower bits of the MQ. However, in an unsigned division the same thing could just as well be accomplished with the following:

$$
\begin{array}{ll}
\text { LAC } & \mathrm{Y} \\
\text { GSM } & \\
\text { DAC }+5 & \\
\text { LAC } \mathrm{X} & \\
\text { CLQ } & \text { / } \rightarrow \text { MQ } \\
\text { LRSS }+5 & \text { /Divide by } 2^{5} \\
\text { DIV } & \\
. . . & / 100 \mathrm{X} / \mathrm{Y} \rightarrow \mathrm{MQ}
\end{array}
$$

This has an advantage in that the instructions CLQ and LRSS +5 can be combined into one instruction through micro programing.

$$
\begin{aligned}
& \text { LAC Y } \\
& \text { GSM } \\
& \text { DAC }+5 \\
& \text { LAC X X } \\
& \text { CLQ! LRSS + } 5 \\
& \text { DIV } \\
& \emptyset \\
& . . . .
\end{aligned}
$$

$1100 \mathrm{X} / \mathrm{Y}+\mathrm{MO}$

Obviously, the quotient $(100 \mathrm{X} / \mathrm{Y})$ can not exceed 1599.99 volts. If it does the link is set as an indication of overflow.

It has been found that if instead of DIVS one uses DIVS-1, the answer is still correct except that it is divided by two. It is possible to take advantage of this when dividing two voltages. In a previous example the properly scaled quotient was located in the MQ by dividing the numerator by $2^{5}$ before the division. Using the DIVS-1 instruction instead of the DIVs instruction a voltage division can be performed as follows:

$\begin{array}{ll}\text { LAC } \quad \mathrm{Y} & \\ \text { GSM } & \\ \text { DAC }+5 & \\ \text { LAC } \mathrm{X} & \\ \text { LRSS }+18^{\circ} & \text { /Load in MQ } \\ \text { LLSS }+14^{\circ} & \text { /Multiply } Y \text { by } 2^{14} \\ \text { DIVS-1 } & \\ . . . & / 100 \mathrm{~K} / \mathrm{Y}+\mathrm{MQ}\end{array}$

One might notice that in the fifth instruction in this example, the numerator is shifted one place further than in the previous example showing division of two voltages. This actually adds about 0.1 microseconds to the program, but the DIVS-1 instruction actually makes a savings of about 0.35 micro-seconds over DIVS. This provides a total savings of about 0.25 micro-seconds in the use of this program.

If one were concerned about time required to perform divisions the following subroutine could be used in place of HDIVS. The calling sequence is altered in that the denominator is loaded into the accumulator first and no attempt is made to detect overflow. This makes tho average time about 42 micro-seconds instead of the 47 micro-seconis for the HDIV\$ subroutine. /CALLING SEQUENCE

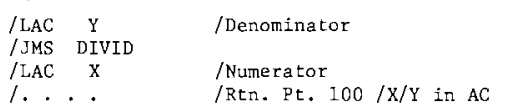

DIVID,
/Rtn. Pt. $100 / \mathrm{X} / \mathrm{Y}$ in $\mathrm{AC}$

$$
\begin{aligned}
& \emptyset \\
& \text { CSM } \\
& \text { DAC . +6 } \\
& \text { XCT I DIVID } \\
& \text { ISZ DIVID } \\
& \text { LRSS I } 8^{\prime} \\
& \text { LLSS } 14^{\prime} \\
& \text { DIVS-1 } \\
& \emptyset \\
& \text { LACQ I DIVID } \\
& \text { JMP I DIVID }
\end{aligned}
$$


K-3

NOTES 
APPENDIX L

DDT DEBUGGING EXAMPLE

This example denonstrates the use of DDT debugging methods and the use of SET 1ines for
ing analog IDACAS ntregrator for

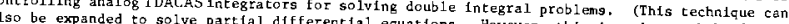
n1que for solving that class of problens.) THE PROBLEY Find the volume enclosed by the paraboloid:

$$
\text { where } \quad \begin{aligned}
& x^{2}+Y^{2}=A^{2} z^{2} \\
& A^{2}=0.1 \\
& 2 \text { ranges from } 0 \text { to } 1
\end{aligned}
$$

PROBLEM ANALYSIS

This is not a practical example because an analytical solution exists.

$$
\text { 1.e. } V=\frac{\pi A^{2} z^{3}}{3}=\frac{\pi A^{2}}{3}=0.10472 \quad(\text { for } z=1)
$$

However, the problem may be solved by an fterative hybrid technique as shoum in Figure 1.

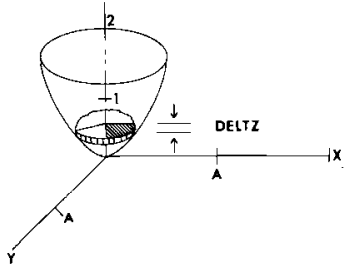

Figure 1

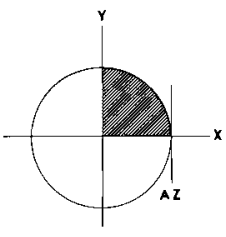

Figure 2
The paraboloid is segmented into a number (i.e., 100) of slabs. (The constant NOITER in the program 1isting is set to 100.) The volune of each slab 19 evaluated by multiplying the

$$
\begin{aligned}
v & =\sum_{\text {DELTZ }=1}^{\text {NOITER }} \text { (DELTZ) (AREA) } \\
& =\text { DELTZ } \sum_{1}^{\text {MITER }} \text { (AREA) } \\
z & =1=(\text { MOITER) (DELTZ) }
\end{aligned}
$$

The slab area show in Figure 2 is evaluated by solving the equation

$$
\mathrm{A}_{\mathrm{z}}=\int_{0}^{\mathrm{A} z} \mathrm{ydx}
$$

(NOTE: The limit of integration is vartable for each slab)

Multiplication of Ay by 4 gives the total area. The volume is then found by

$$
\mathrm{V}=4 \text { (DELIZ) } \sum_{1}^{\text {NOITER }} \int_{0}^{A Z} \sqrt{\mathrm{A}^{2} z^{2}-\mathrm{x}^{2}} \mathrm{dx}
$$

AMPLITUDE SCAL.TNG

Scaling factors of 100 for $\mathrm{A}^{2}, 100$ for $\mathrm{z}^{2}$, and 1000 for JeLtz seem reasonsble because

$$
100 \mathrm{~A}^{2}=10
$$$$
1002^{2}+\text { ranges from } 0 \text { to } 100
$$

1000 DELTZ $=10$

\section{SIMULATION DIAGRAMS}

Figures 3 and 4 show analog and digital logic dlagrams for solving the problem. Figure 5 contains the listing (nistakes are circled), assembler error nessages, end the are those typed by the user. The symbols $t$ and 0 are Teletype line feed and carriage teturn characters also typed by the user. Column 3 explains the debugging procedure.

ACCURACY

Since accuracy considerations of analog and hybrid computers are "shadowed" by false impressions, it is worthwh1le explaining the $3 \%$ answer obtatned in this problem. distorted accuracy opinions 3\% accuracy 15 all one can expect vith an analog computer.)

The solution of this problem by fterative techniques required 100 open ended integrations. To achieve the overal1 accuracy of 38 each integration must be aceurate to
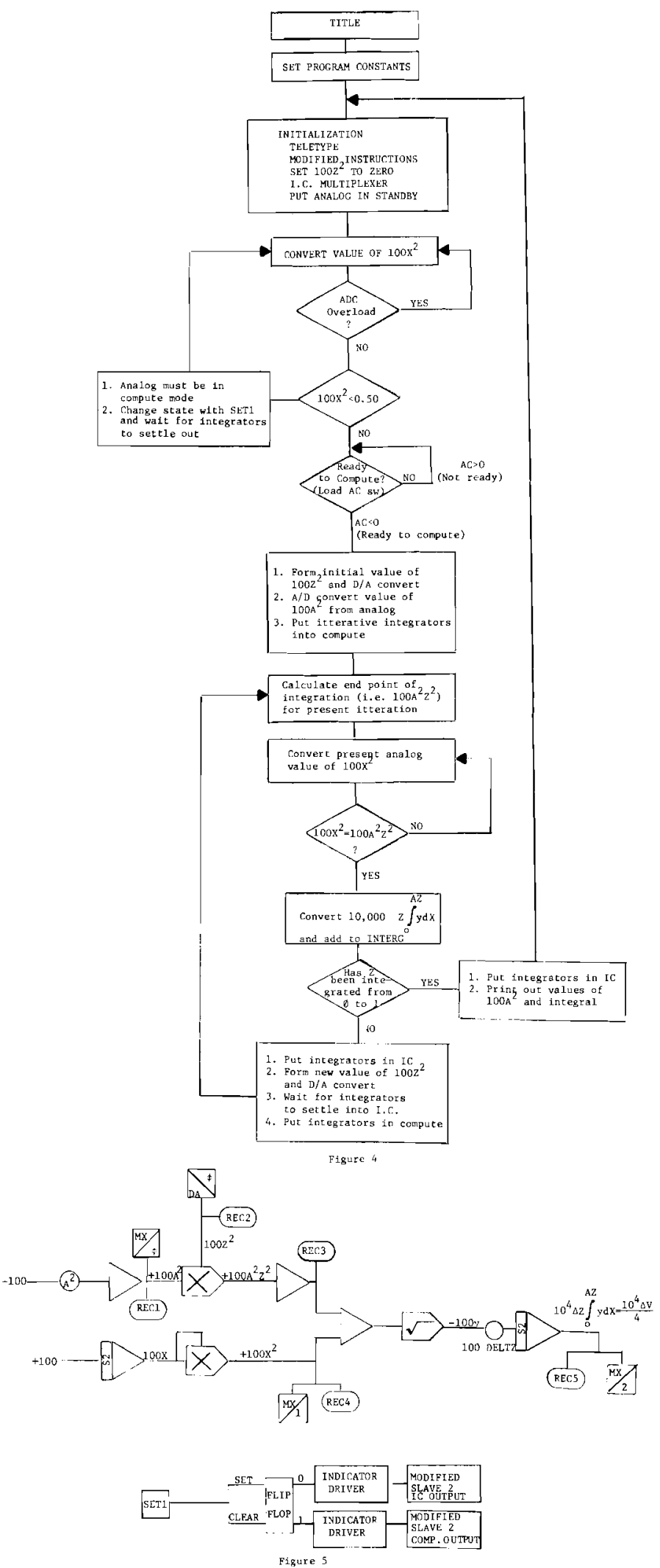

LOGIC FOR CONTROL OF IDICAS INTEGRATORS 


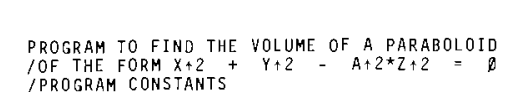

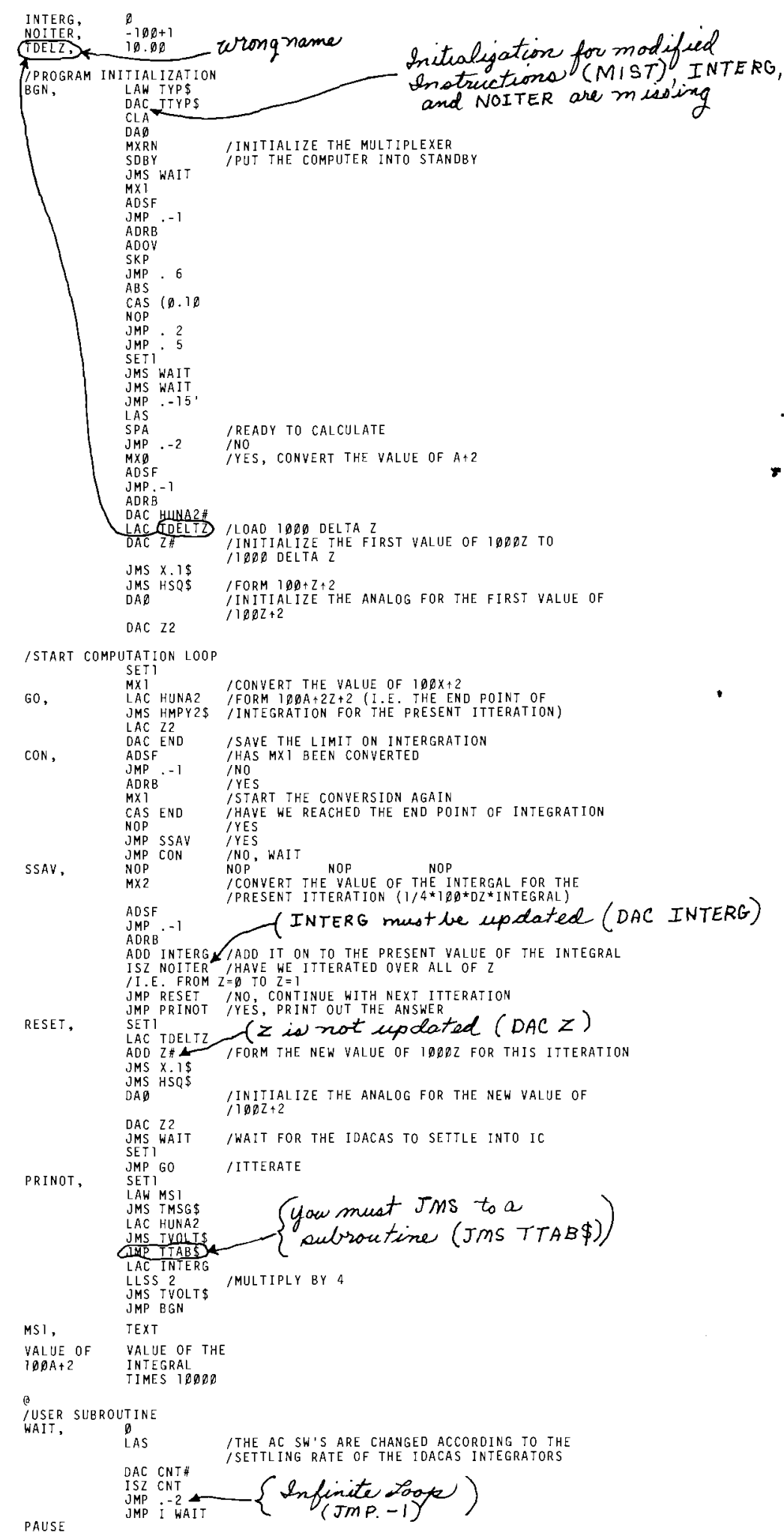

ASSEMBLER ERROR MESSAGES

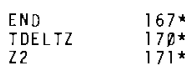

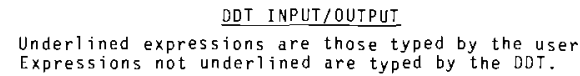

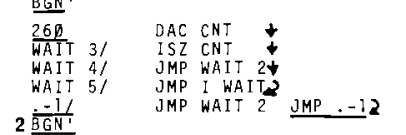

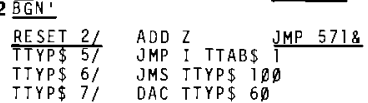

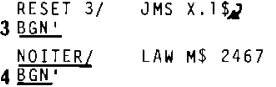

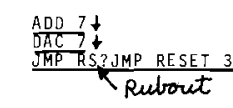

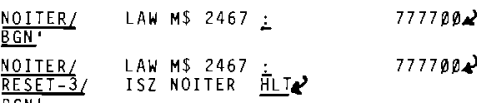

SETI 先

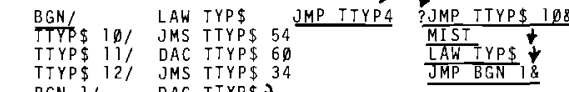

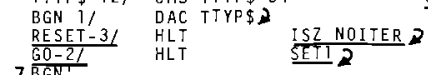

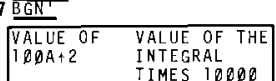

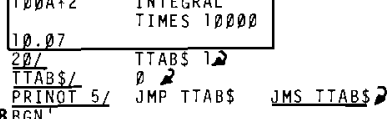

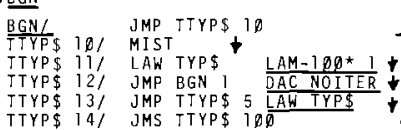

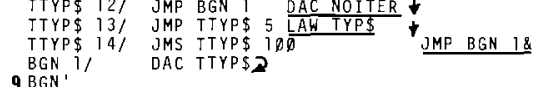

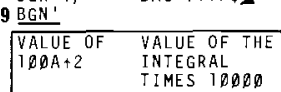

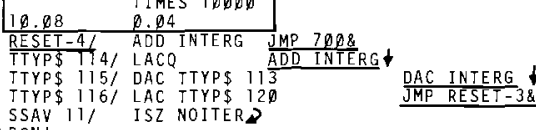

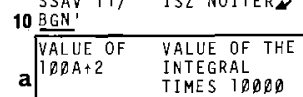

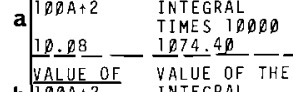

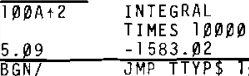

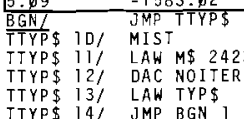

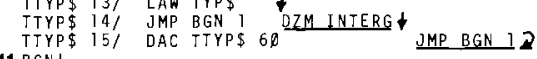

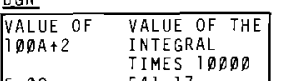

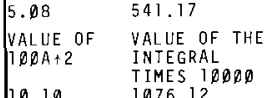

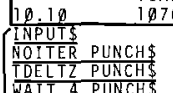

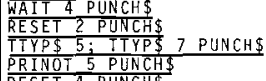

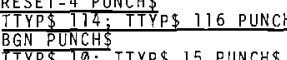

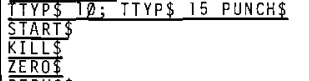

\begin{tabular}{lll} 
& 6 & 13467 \\
\hline OADS & 693 & 13467
\end{tabular}

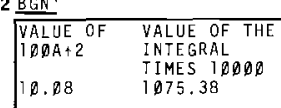

-

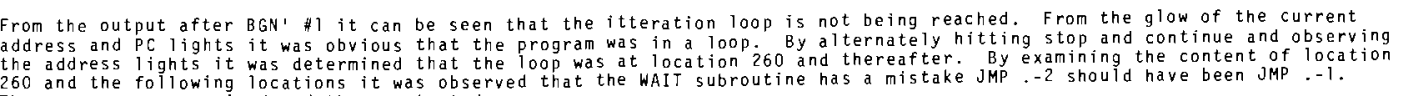

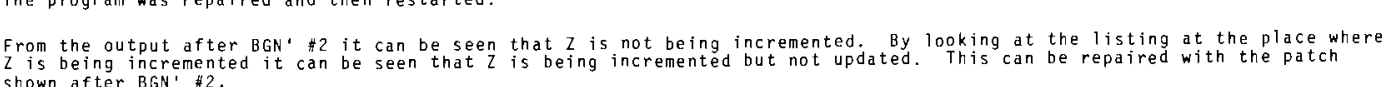

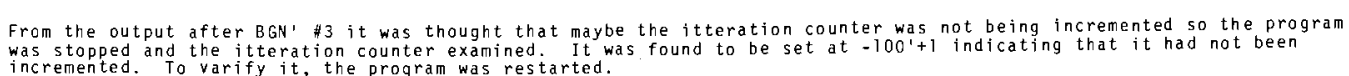

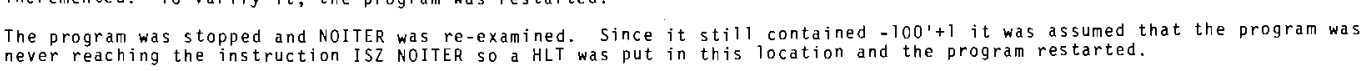

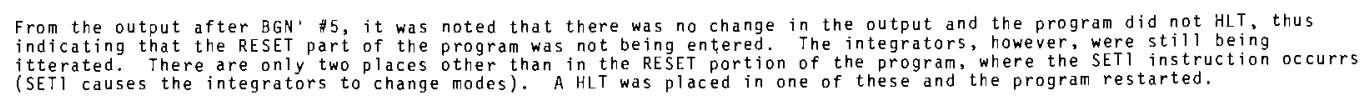

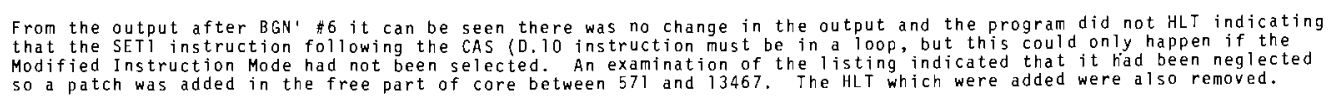

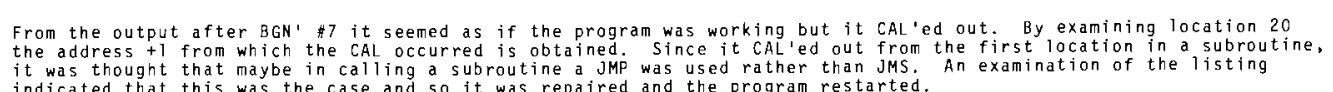

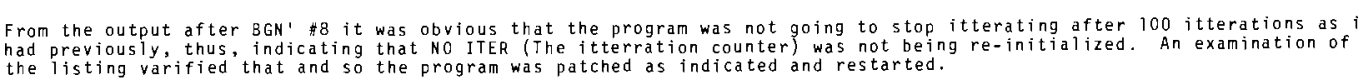

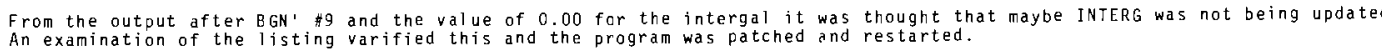

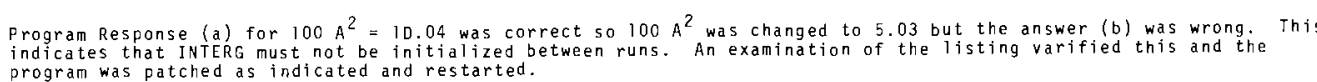

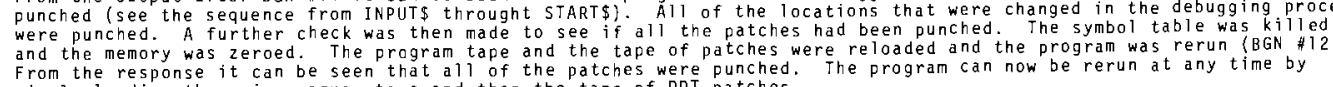


$\mathrm{A}^{2}$
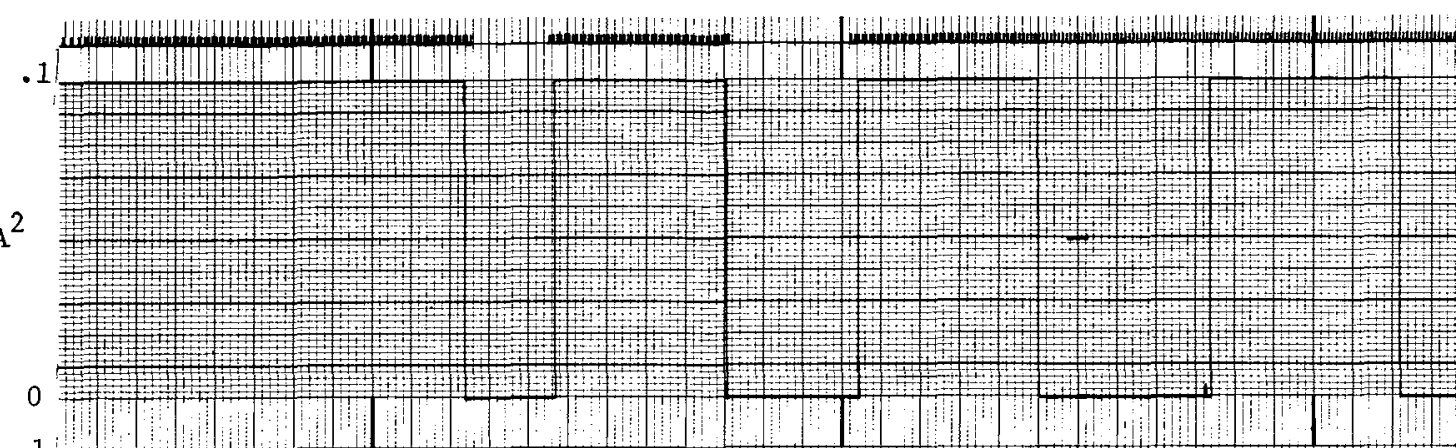

$z^{2}$ .

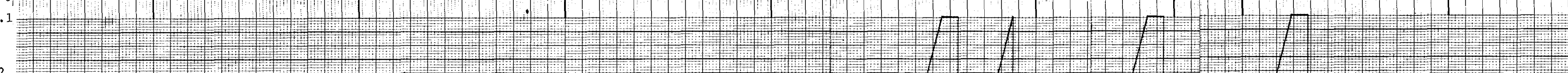

年

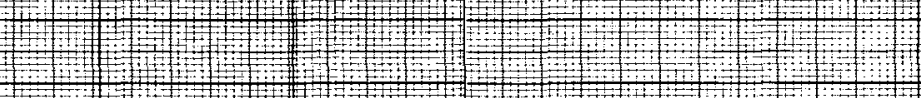

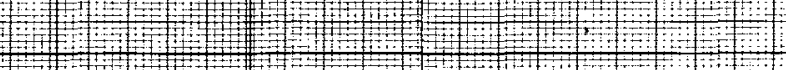

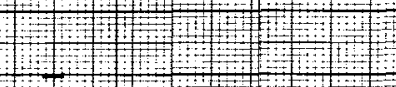

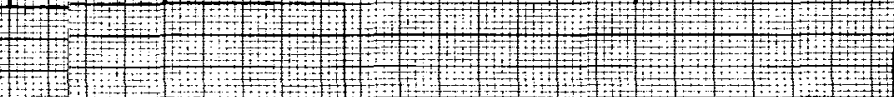

$\mathrm{A}^{2} \mathrm{z}^{2}$

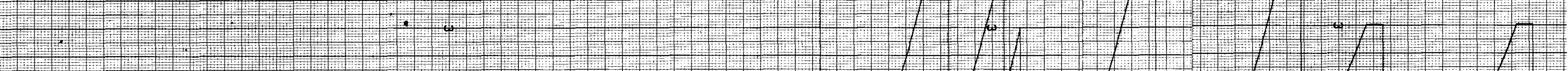

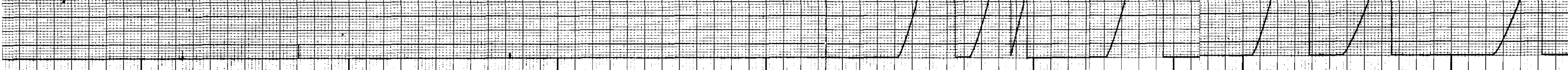

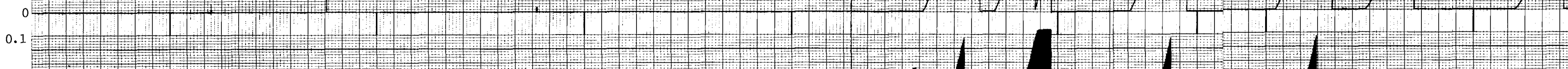

$\mathrm{x}^{2}$

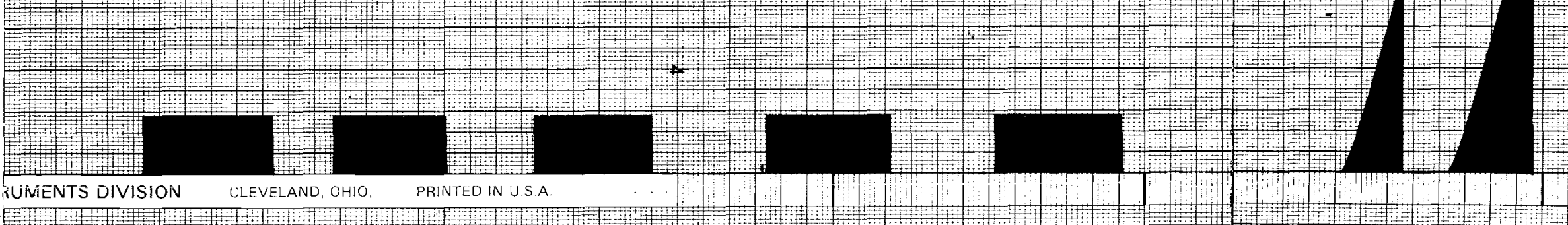

$\Delta 2 \int_{0}^{\mathrm{AZ}} \mathrm{yd}$ 
DDT DEBUGGING EXAMPLE (contd)

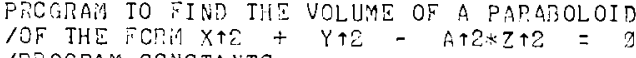

$\begin{array}{ll}\text { INTERG, } & 5 \\ \text { NOITER; } & -19 \pi^{\circ} \\ \text { TDELTZ, } & \text { 19. }\end{array}$

MPRCCFAIA INI TIALIZATION

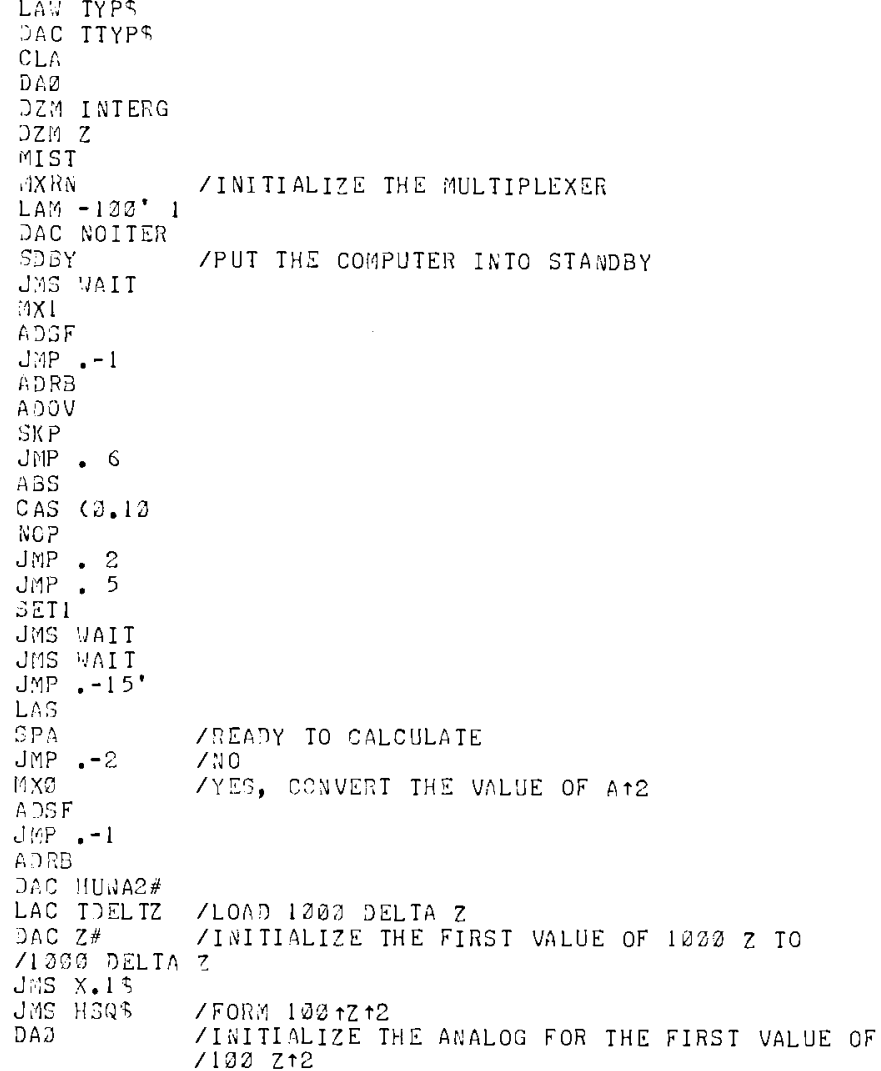

JAC 22
ISTART COMPLIATION LOOP

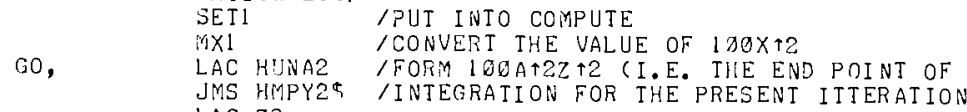

CON, JACEND SSAVE THE LIMIT ON INTERGRATION

ADSF -1 HAS MX1 BEEN CONVERTED

$\operatorname{JMPPB}{ }^{-}-$

XI

CAS END

JMP SSAV

YYES

JTAART THE CONVERSTOA AGAIN

THAVE WE REACHED THE END POINT OF INTEGPATION

YYES

SSAV, NOP CON SNOP VAIT THE VALUE OF THE INTERGAL FOR THE

NOP

NOP
NOP
NOP

ADSF

$M P P^{-1}$

AND INESG /ADS IT ON TO THE PRESENT VALUE OF THE INTEGRGL ISZ NOITER

/HAVE NE ITTERATED OVER ALL OF $Z$

JMP RESET SNOE, CONTI IUE WI TH WEXT ITTERATION

F.ESET,

SET1 $L A C$ TDELTZ

ADD Z Z /FORII THE NEN VALUE OF 1 IgOdZ FOR THIS ITTERATION

DAC 2

JMS $X_{0} \cdot 19$

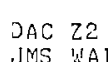

INIIIA
MOIZT2

JMS "AIT /WAIT FOR THE iDACAS TO SETTLE INTO IC

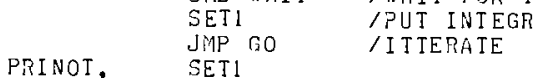

SET1

LAW
JMS TISTSS

LAC HUNAZ

JMS TTABS

LLSS 2

JMS IVOL
JMP BGN

IIS1

VALUE OF VALUE OF THE

IDTEGRTAL

IJJER SUEROUTINE

WAIT, LAS

/IHE AC SW'S ARE CHANGED ACCORDING TO THE
/SETTLING RATE OF THE IDACAS INTEGRATORS

DAC CNIF
ISZ CNI

$\underset{\text { JiAP }}{-1}$ I WAI $^{-1}$

WAIT, LAS

Lis

SAC CNTH
ISZ CNT
JMP

MULTIPLY BY 4

JMPP $\dot{I}^{-1}$ WAII
PRJSRA Y IJ FI:D THE VOLUAE OF A PARAGJLOID

$175 \%$
72

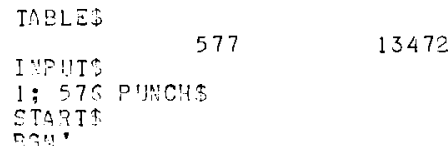

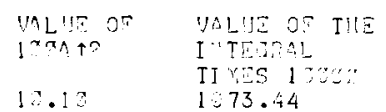

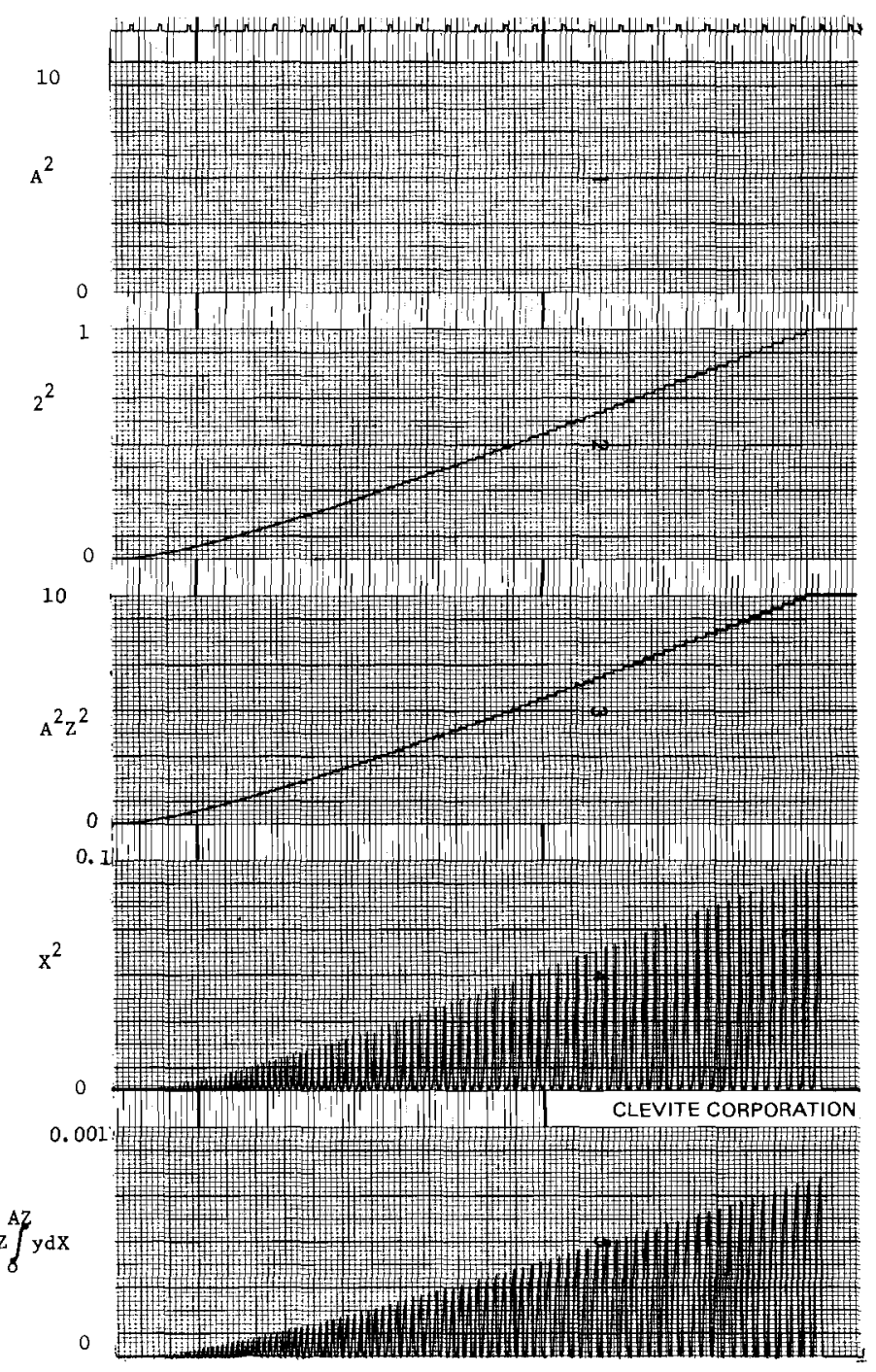

蛋

$\underset{\infty}{\infty}$ 
PILOT EJECTION STUDY

SIMPL-1 HYBRID SOLUTION FOR THE PILOT EJECTION STUD

thetement for this problem is taken from SBG-TDR-64-1. "MIDAS Progranning Guide". The purpose of this investigation ts to determine the trajectory of a pilot ejected from a fighter aircraft in order to ascertain whether he will strike the vertical stabilizer of the drag on the p1lot, causing his relative horizontal motion with respect to the aircraft,

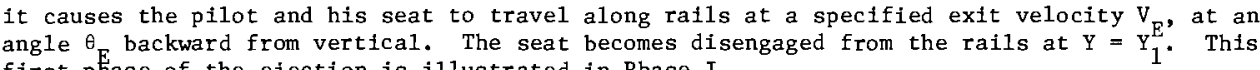

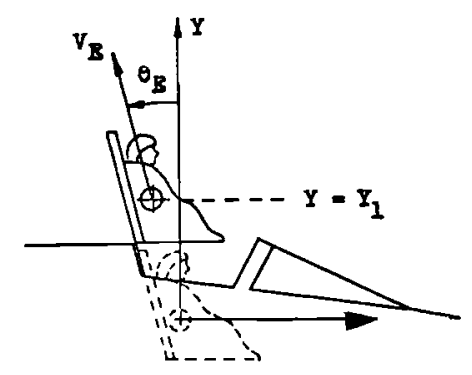

PHASE I

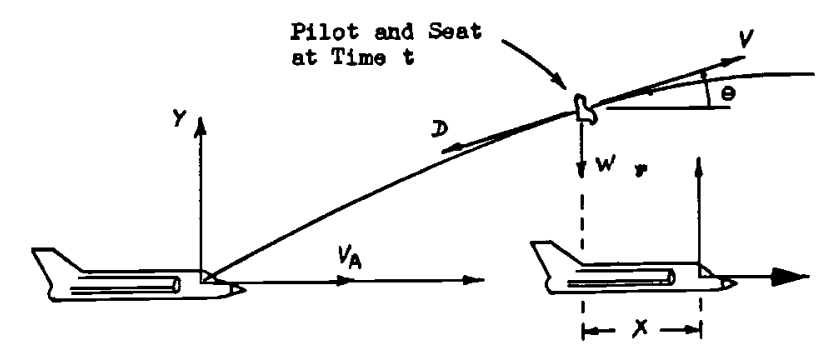

PHASE II
Once the pilot and seat combination leaves the rails, it follows a ballistic trajectory to the aircraft (which is assmed to fly level at constant speed) that is important, we can formulate of

\section{The governing equations are}

$\dot{\mathrm{x}}=\mathrm{v} \cos \theta-\mathrm{v}$

$\dot{\mathrm{y}}=\mathrm{v} \sin \theta$

$\dot{\mathrm{v}}=0$

$=-\frac{D}{m}-g \sin \theta$

$\dot{\theta}=0$

$=-(g \cos \theta) / \mathrm{V}$

$\mathrm{D}=\frac{1}{2^{2} \mathrm{C}_{\mathrm{d}} \mathrm{Sv}^{2}}$
Constants

$\mathrm{m}=7$ slugs

$\mathrm{g}=32.2 \mathrm{ft} / \mathrm{sec}^{2}$

$\leq \mathrm{Y}<\mathrm{Y}_{1} \quad \mathrm{C}_{\mathrm{d}}=1$

$0 \leq Y<Y_{1} \quad \mathrm{Y}_{1}=4 \mathrm{ft}$

$\mathrm{Y} \geq \mathrm{Y}_{1} \quad \mathrm{~V}_{\mathrm{E}}=40 \mathrm{ft} / \mathrm{sec}$

$\theta_{\mathrm{E}}=15^{\circ} \quad(=15 / 57.3$ radians $)$

$\mathrm{V}_{\mathrm{A}}=900 \mathrm{ft} / \mathrm{sec}$

$\rho=2.3769 \times 10^{-3} \mathrm{slugs} / \mathrm{ft} \mathrm{t}^{3}$
The initial values of $\mathrm{V}$ and $\theta$ (pilot's initial velocity vector at moment of leaving
cockpit rails) are given by:

$$
\begin{aligned}
& V(0)=\sqrt{\left(V_{A}-V_{E} \sin \theta_{E}\right)^{2}+\left(V_{E} \cos \theta_{E}\right)^{2}} \\
& \theta(0)=\tan ^{-1} \frac{V_{E} \cos \theta_{\mathrm{E}}}{V_{A}-V_{E} \sin \theta_{E}}
\end{aligned}
$$$$
\mathrm{X}(0)=\mathrm{Y}(0)=0
$$

A run is to be terminated when any one of these conditions occurs

$\mathrm{X} \leq-60$ feet (pilot beyond vertical stabilizer)

$\mathrm{Y} \geq 30$ feet (pilot well above $12 \mathrm{ft}$. high tail)

$t \geq 4.0$ secs.

$v, v, \theta, X$, and $Y$ vs. time

P1ot $X$ vs.

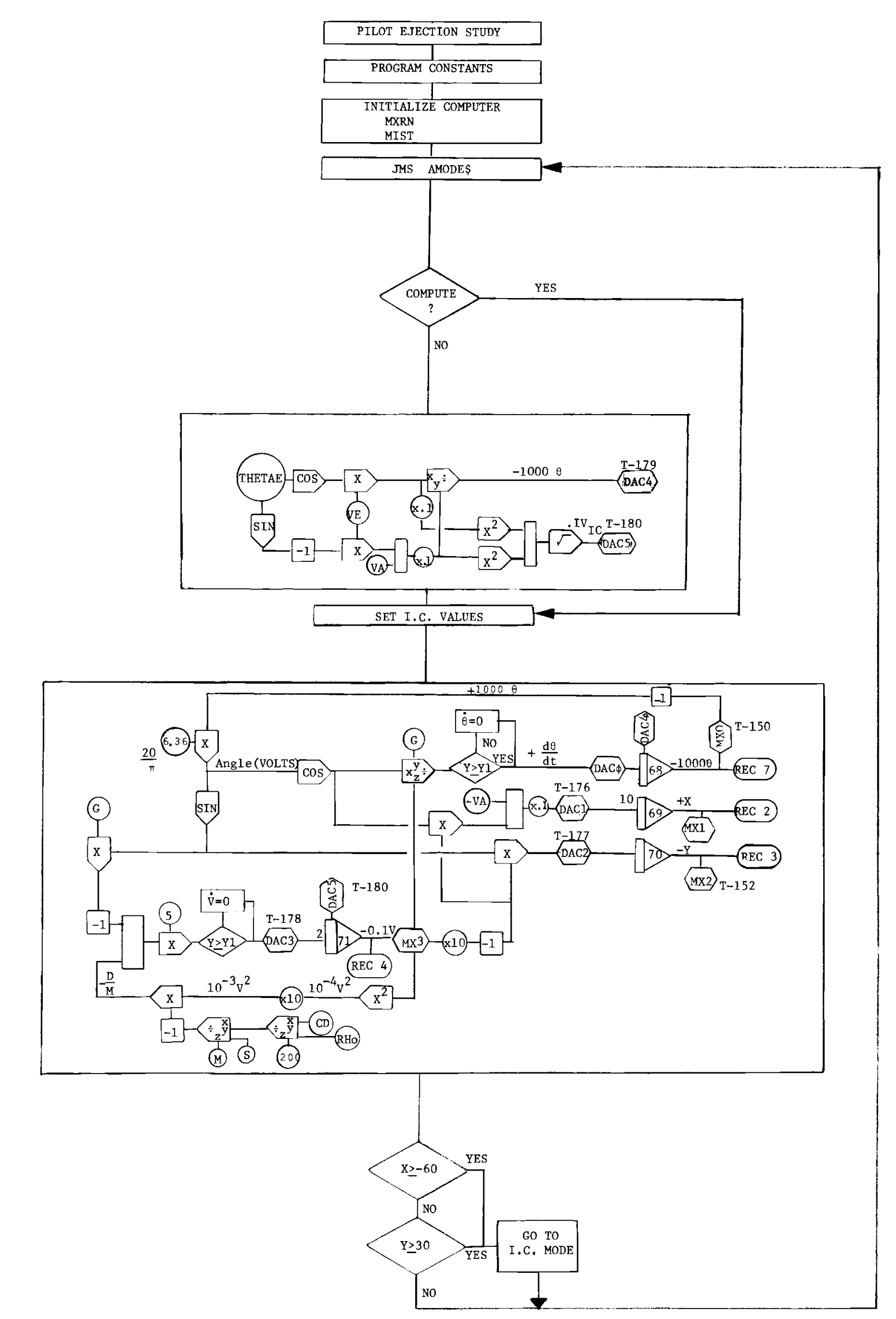




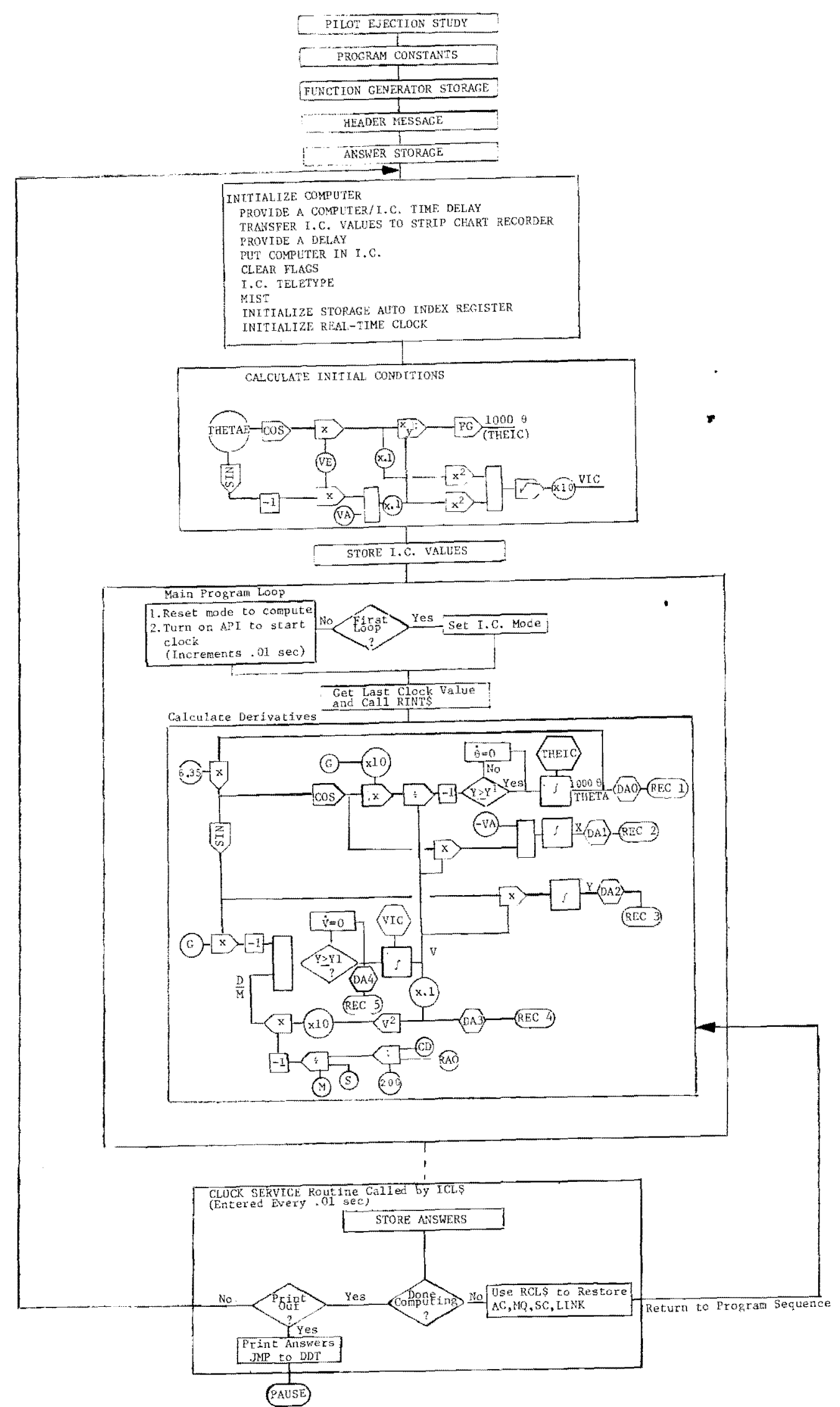

PRINTOUT FOR REAL TIME SOLUTION (A TOTAL OF 331 COMPUTING FRAMES WERE USED)

VA RHO

$90.00 \quad 237.70$

\begin{tabular}{|c|c|c|c|c|c|}
\hline $\begin{array}{l}\text { TIME } \\
(X \perp 00 \quad S E C)\end{array}$ & VDOT & v & THETA & $x$ & $Y$ \\
\hline 6) & 0.00 & 890.38 & 43.52 & 0.00 & $0 . \% ?$ \\
\hline 1 & 0.00 & 890.39 & 43.52 & -0.09 & 0.35 \\
\hline 2 & 0.00 & 890.33 & 43.52 & -8.18 & 9.71 \\
\hline 3 & 0.00 & 890.38 & 43.52 & -0.29 & 1.11 \\
\hline 4 & 0.00 & 898.38 & $43.5 ?$ & -0.40 & 1.51 \\
\hline 5 & 0.00 & $89 n .38$ & $43.5 ?$ & -0.50 & 1.87 \\
\hline 5 & 0.20 & 890.38 & 43.52 & -9.61 & 2.28 \\
\hline 7 & 0.00 & 890.30 & 43.52 & -0.71 & 2.54 \\
\hline 8 & 0.20 & 890.38 & 43.52 & $-x .92$ & 3.84 \\
\hline 8 & 0.00 & 890.38 & 43.50 & -.93 & 3.44 \\
\hline 10 & 9.00 & 890.33 & 43.52 & -1.21 & $3.0 \%$ \\
\hline 11 & -1331.77 & 883.42 & 43.33 & -1.15 & $4.2 \pi$ \\
\hline 12 & -1290.31 & 371.36 & 42.94 & -1.36 & 4.55 \\
\hline 13 & -1255.27 & 857.87 & $4 ? .60$ & -1.72 & 4.24 \\
\hline 14 & -1215.22 & 346.25 & 42.26 & -2.23 & 5.29 \\
\hline 15 & -1185.19 & 833.52 & 41.25 & -2.31 & 5.65 \\
\hline 16 & -1150.57 & 822.78 & 41.43 & $-3.5 ?$ & 5.97 \\
\hline 17 & -1120.71 & 812.52 & 41.85 & -4.37 & 6.34 \\
\hline 18 & -1388.57 & 860.35 & 47.54 & -5.39 & 3.69 \\
\hline 19 & -1951.21 & 788.97 & 40.27 & -6.36 & 5.97 \\
\hline 26 & -1031.55 & 777.88 & 39.83 & -7.59 & 7.39 \\
\hline 21 & -1003.36 & 763.32 & 39.44 & -3.79 & 7.53 \\
\hline 22 & -979.71 & 757.31 & 38.99 & $-12 \cdot 24$ & 7.91 \\
\hline 23 & -953.33 & 743.92 & 38.62 & -11.59 & 8.13 \\
\hline 24 & -938.77 & 738.93 & 38.15 & -13.24 & $8.4 \%$ \\
\hline 25 & -906.52 & 730.30 & 37.73 & -14.20 & 8.74 \\
\hline 26 & -886.19 & 720.30 & 37.27 & -16.64 & 9.72 \\
\hline 27 & -863.37 & 712.74 & 36.79 & -18.32 & 9.26 \\
\hline 28 & -344.29 & 703.70 & 36.36 & -20.36 & 9.55 \\
\hline 29 & -823.13 & 695.36 & 35.88 & -22.49 & 9.78 \\
\hline 30 & -305.51 & 687.23 & 35.45 & -24.45 & 10.04 \\
\hline 31 & -735.60 & 679.91 & 34.95 & -26.76 & 17.29 \\
\hline 32 & -769.20 & 671.67 & $34.5 !$ & -28.32 & 10.51 \\
\hline 33 & -750.54 & 664.53 & 33.98 & -31.34 & 10.76 \\
\hline 34 & -734.96 & 556.65 & 33.55 & -33.53 & 10.96 \\
\hline 35 & -717.75 & 648.96 & 33.92 & -36.16 & 11.15 \\
\hline 36 & -703.23 & 642.30 & 32.55 & -38.55 & 11.39 \\
\hline 37 & -687.46 & 634.94 & 32.82 & -41.32 & 11.01 \\
\hline 38 & -671.91 & 628.69 & 31.54 & -43.79 & 11.79 \\
\hline 39 & -658.62 & 621.63 & 31.02 & -46.71 & $12 . x$ \\
\hline 40 & -644.099 & 615.52 & 39.52 & -49.34 & 12.17 \\
\hline 41 & -631.85 & 608.75 & 29.96 & -52.39 & 12.37 \\
\hline $4 ?$ & -617.95 & $6 \times 3.68$ & 29.41 & -55.46 & 12.54 \\
\hline 43 & -606.52 & 596.51 & 28.90 & -53.29 & 12.72 \\
\hline 44 & -593.56 & 597.99 & 28.32 & -61.54 & 10.99 \\
\hline
\end{tabular}


SIMPL-1 ALL DIGITAL SOLUTION FOR THE PILOT EJECTION STUDY (contd)

\section{DISCUSSION OF SIMPL-1 (ALL DIGITAL) PILOT EJECTION STUDY}

The problem definition, flow charts, computer program and output are contained on the preceding pages.

This example is significant because it demonstrates:

1. Amplitude scaling

2. Use of function generator programs

3. The format and method of printing header messages on Teletype

4. Method of storage allocation for numeric printout

5. User subprograms and computer initialization

6. The ICL\$ (initialize real-time clock) and the corresponding programs serviced by the automatic priority interrupt

7. Pre-calculation of initial conditions

8. Auto index registers for answer storage and retrieval

9. The main computing loop and subroutine RINT\$

10. The use of the teletype library for printing out answers.

\section{SCALING}

Since this is an all digital simulation, all values should be scaled between \pm 10 (or 50 ) and \pm 1599.99 . On a large complex problem scaling information could be obtained by first writing an APACHE (5) or MIDAS (6) program. Since this is a relatively simple problem, amplitude scaling can be accomplished by inspection. From equations and constants given in the problem definition, it is obvious that $\theta, M, C_{d}, S, V^{2}, p$ and the squared terms in the $V(0)$ equation require amplitude scaling. (A scale factor for the switching variable Y1 is not needed.) For convenience the following relationships (showing scaling factors) are chosen.

$$
\begin{aligned}
& 1000 \theta \simeq 400 \\
& 10 \mathrm{M}=70 \\
& 100 \mathrm{C}_{\mathrm{d}}=100 \\
& 10 \mathrm{~S}=100 \\
& 10^{-4} \mathrm{~V}^{2}=81 \\
& 10^{5} \rho=273.69 \\
& 100 \cos \theta \simeq 100 \\
& 100 \sin \theta \simeq 4.0 \\
& \left\{0.1 \mathrm{~V}_{\mathrm{A}}-0.1 \mathrm{~V}_{\mathrm{E}} \sin \theta\right\} \simeq 81 \\
& 0.1 V_{\mathrm{E}} \cos \theta \simeq 4
\end{aligned}
$$

(scaling for this term is required for compatibility with other terms in $\mathrm{V}(0)$ calculation) 
SIMPL-1 HYBrid SOLUTION FOR THE PILOT EJeCtion STUDY (Contd)

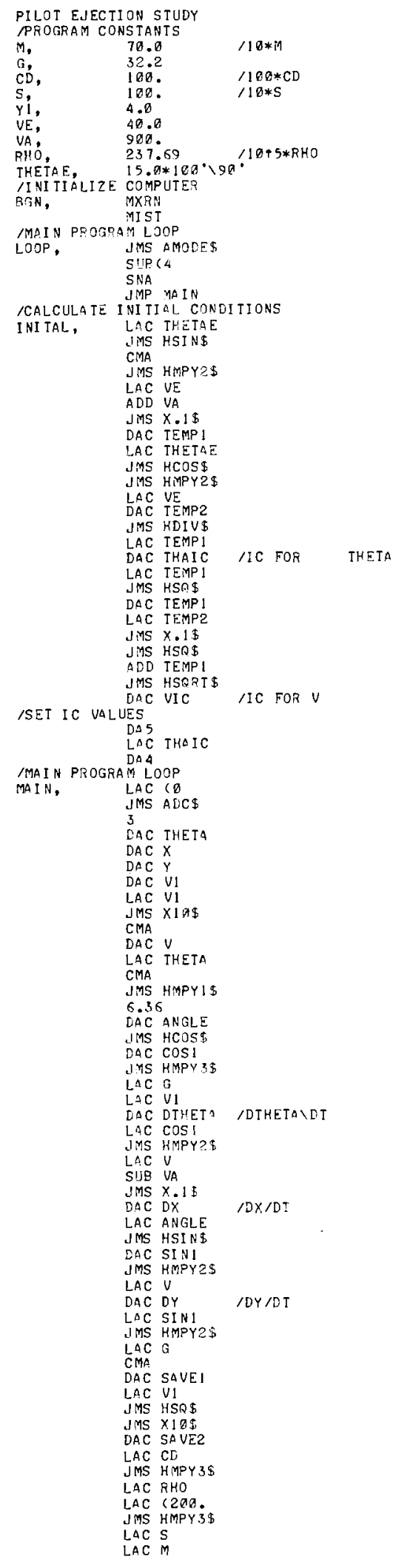

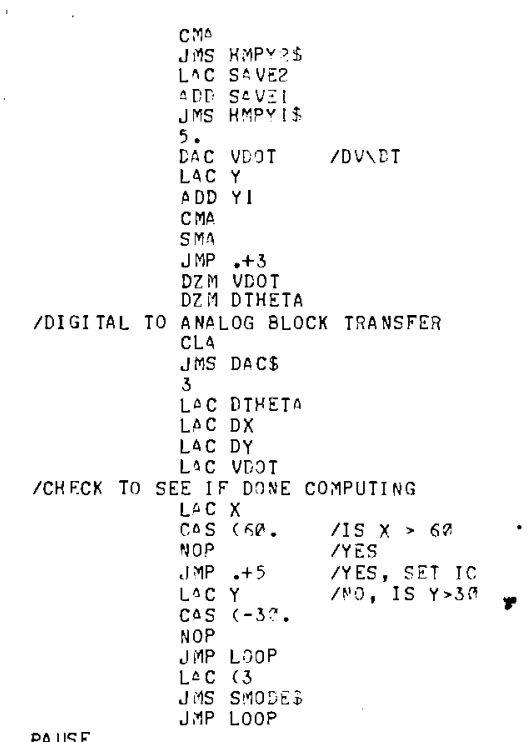

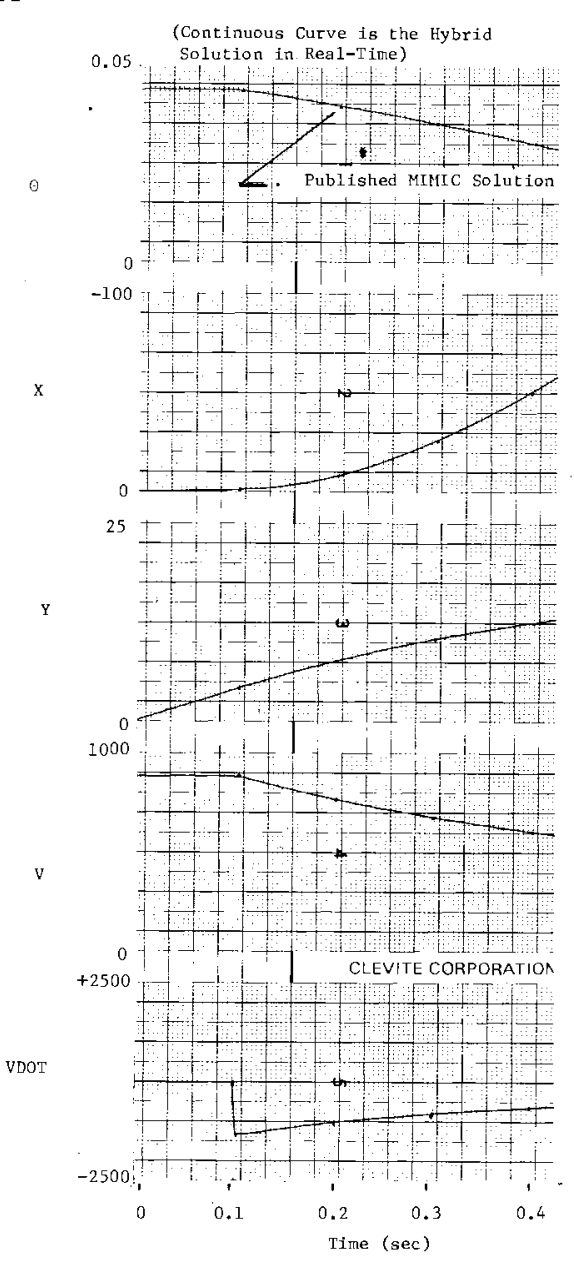

DISCUSSION OF THE SIMPL-1 (hybrid) PILOT EJECTION SIMULATION

Aotice on the illustrations to the left that every program must have:

1. A title

. A tabulation of program constants

3. A computer initialization section

4. A main program loop for

(a) mode checking

initial conditions (optiona1)

(c) analog to digital transfer

(e) digital to analog tran fe

(f) computing termination test (optional)

5. A PAUSE statement

IITLE

The titie for the 1isting is "Pilot Ejection Study.

IABULATION OF PROGRAM CONSTANTS

The slash (/) in the 1 ine "/Program Constants" is an assembler comments character. If a carriage return $(\mathcal{H})$ or tab $(\rightarrow \mid)$ preceeds the slash $(/)$, the assembler ignores all information following the slash until the next carriage return.

Notice the utility of the slash in comments for $M, C D, S$, and RHO. The /1ø*M informs the value of $M$ (which is 7). Numerical values of all program constants and variables must contain a decimal point to signify voltage scaling see Appendix C).

The 1isting for THETAE demonstrates a useful feature of the SIMPL-1 assembler for preforming mathematical operations. The sequence of operations $\left(15^{*} 19 \phi^{\prime} \backslash 9 \phi^{\prime}\right)$ are used to convert THETAE fro COMPUTER INITIALIZATION

In this example the modified instructions and random select multiplexer modes are specified.

\section{MAIN PROGRAM LOOP*}

The first function performed by the main computing loop is to check the analog computers

mode. The initial condition calculations are entered for every analog computer mode except compute. The I.C. values are transferred to the analog computer through use of assembly language digital to nalog conversion instructions.

The derivative calculation loop begins with the analog to digital block transfer calling

sequence. The derivative calculations are then accomplished by interconnecting mathematical build $\mathrm{d} / \mathrm{dt}$ and $\mathrm{dV} / \mathrm{dt}$ are set to zero is made prior to digital to analog block transfer of derivatives. The 1ast function of the probran is to petermine if $x$ and $Y$ have exceeded end of run linits. If not, the derivative calculations are repeated.

PAUSE

The last statement of any STMPL-1 program should be the pseudo-instruction PAUSE, GENERAL REMARKS ABOUT THE PROGRAM

The program as written required 760 memory locations (or $9.3 \%$ of the computers 8192 memory 1ocations) and adequately simulated the problem ten times faster than real-time.

The derivative calculation frame time is about 1100 to $1200 \mathrm{\mu sec}$. This could be reduced to about 800 usec by replacing analog to digital and digital to analog block transfers with assemb1y language 1istings, Further special improvements could be made by performing the calculation for $10^{5} \mathrm{SC}_{\mathrm{d}} /(2 \mathrm{M})$ only once in the initialization section.

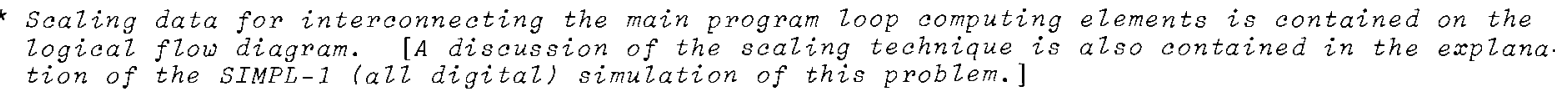


SIMPL-I ALL DIGITAL SOLUTION FOR THE PILOT EJECTION STUDY (contd)

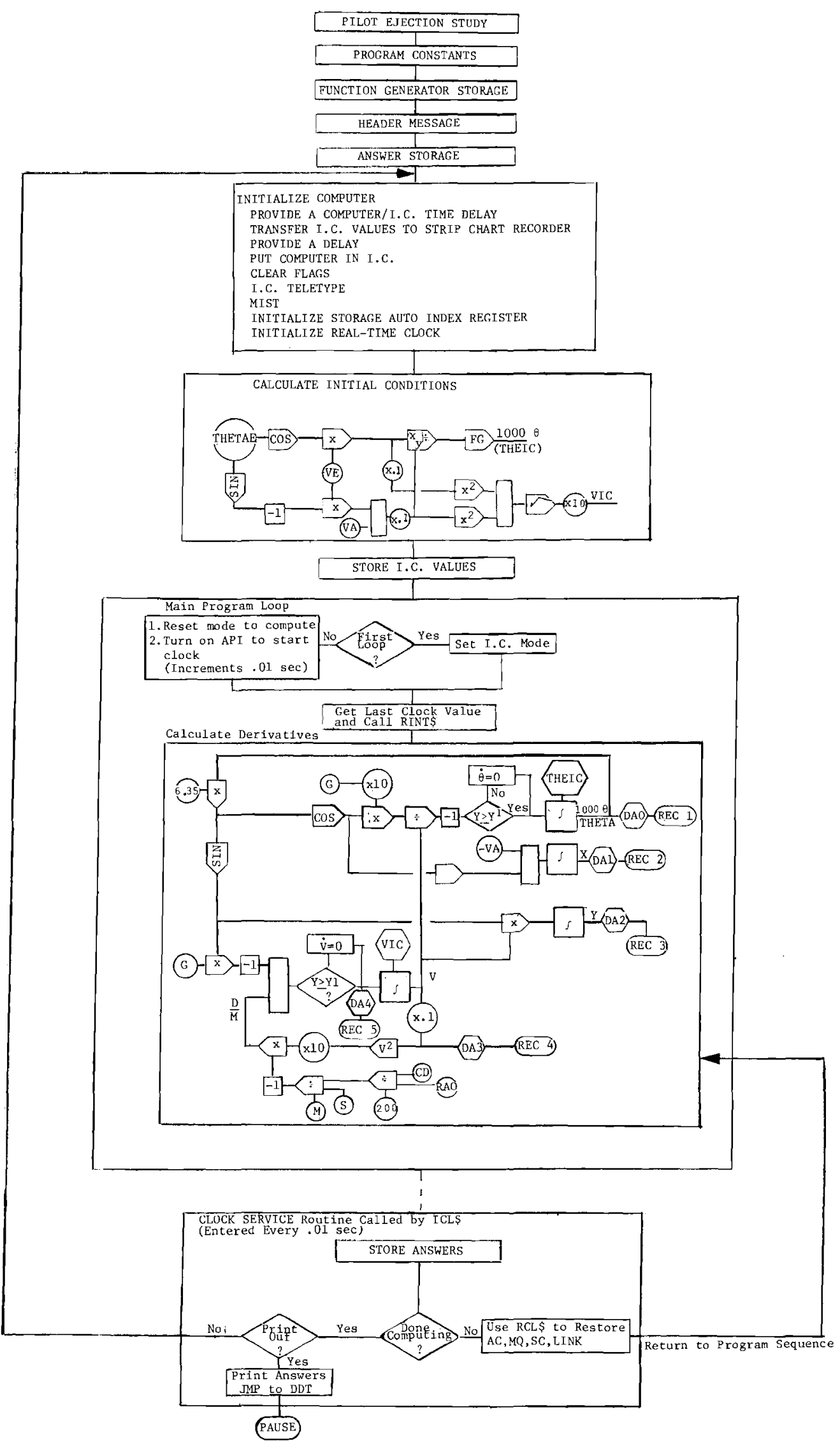


SIMPL-1 ALL DIGITAL SOLUTION FOR THE PILOT EJECTION STUDY (contd)

DISCUSSION OF SIMPL-1 (ALL DIGITAL) PILOT EJECTION STUDY

The problem definition, flow charts, computer program and output are contained on the preceding pages.

This example is significant because it demonstrates:

1. Amp1itude scaling

2. Use of function generator programs

3. The format and method of printing header messages on Teletype

4. Method of storage allocation for numeric printout

5. User subprograms and computer initialization

6. The ICL\$ (initialize real-time clock) and the corresponding programs serviced by the automatic priority interrupt

7. Pre-calculation of initial conditions

8. Auto index registers for answer storage and retrieval

9. The main computing loop and subroutine RINT\$

10. The use of the teletype library for printing out answers.

\section{SCALING}

Since this is an all digital simulation, all values should be scaled between \pm 10 (or 50) and \pm 1599.99 . On a large complex problem scaling information could be obtained by first writing an APACHE (5) or MIDAS (6) program. Since this is a relatively simple problem, amplitude scaling can be accomplished by inspection. From equations and constants given in the problem definition, it is obvious that $\theta, M, C_{d}, S, V^{2}, \rho$ and the squared terms in the $V(0)$ equation require amplitude scaling. (A scale factor for the switching variable $\mathrm{Y} 1$ is not needed.) For convenience the following relationships (showing scaling factors) are chosen.

$$
\begin{aligned}
& 1000 \theta \simeq 400 \\
& 10 \mathrm{M}=70 \\
& 100 \mathrm{C}_{\mathrm{d}}=100 \\
& 10 \mathrm{~S}=100 \\
& 10^{-4} \mathrm{~V}^{2}=81 \\
& 10^{5} \rho=273.69 \\
& 100 \cos \theta \simeq 100 \\
& 100 \sin \theta=4.0 \\
& \left\{0.1 V_{\mathrm{A}}-0.1 \mathrm{~V}_{\mathrm{E}} \sin \theta\right\} \simeq 81 \\
& 0.1 V_{\mathrm{F}} \cos \theta \simeq 4
\end{aligned}
$$


The applicability of the above scale factor can be tested as follows: Since a unity scale factor for $V$ is acceptable the scale factors selected for $\rho, C_{d}, S$, and $V^{2}$ should produce unity...but will they?

$$
\begin{aligned}
& \frac{D}{M}=\frac{\rho C_{d} S V^{2}}{2 M} \\
& {[1] \frac{D}{M} \neq \frac{1}{2} \frac{10^{5} \rho\left[\begin{array}{lll}
100 & \left.C_{d}\right]\left[\begin{array}{ll}
10 & S
\end{array}\right]\left[10^{-4} V^{2}\right]
\end{array}\right.}{\left[\begin{array}{lll}
10 & M
\end{array}\right]}}
\end{aligned}
$$

to make the equation compatible the right side must be divided by 1000 .

$$
\text { [1] } \frac{\mathrm{D}}{\mathrm{M}}=\frac{1}{2} \frac{10_{\rho}^{5}\left[\begin{array}{lll}
100 & \mathrm{C}_{\mathrm{d}}
\end{array}\right]\left[\begin{array}{ll}
10 & \mathrm{~S}
\end{array}\right]\left[10^{-4} \mathrm{~V}^{2}\right]}{[1000]\left[\begin{array}{ll}
10 & \mathrm{M}
\end{array}\right]}
$$

The value of $\mathrm{D} / \mathrm{M}$ is calculated in four steps. (See the main computing loops block diagram.) First the ratio $\left(100 \mathrm{C}_{\mathrm{d}}\right)\left(10^{5} \mathrm{p}\right) / 200$ is evaluated with a HMPY3\$ routine. The second operation involves multiplying this ratio by $10 \mathrm{~S} / 10 \mathrm{M}$ with another HMPY 3 routine to produce a scale factor of $10^{5}$. The third operation involves squaring $0.1 \mathrm{~V}$ with a HSQ $\$$. The resulting scale factor is $10^{-4} \mathrm{~V}^{2}$ because the hybrid squaring routine automatically multiplies the result by $10^{-2}$. A scale factor of $10^{-3}$ for $V^{2}$ is obtained by multiplying by 10 . The final ratio for $\mathrm{D} / \mathrm{M}$ is obtained by multiplying $-10^{5}\left[\mathrm{C}_{\mathrm{d}} \rho \mathrm{S}\right] /[2 \mathrm{M}] \mathrm{by}$ $10^{-3} V^{2}$ with a HMPY $2 \$$ routine. The resulting scale factor for $D / M$ is 1 because the HMPY $2 \$$ automatically divides by an additional factor of 100 .

The proper scale factor of 1000 for $\theta$ is obtained in the main computing loop by multiplying $G$ by 10 and taking advantage of the 100 multiplying scale factor associated with the hybrid divide routine. Note the necessity of converting $1000 \theta$ (in radians) to volts by multiplying by $0.1\left(\frac{200}{\pi}\right.$ ) before evaluating the sin and cos functions. In the initial conditions calculation the scale factor of 1000 for $\theta(0)$ is also obtained by taking advantage of the HDIV $\$$ and problem variable scale factors. The mechanism for this is left as a reader exercise.

There are two reasons for using a function generator to evaluate the initial condition for $1000 \theta$ (or THEIC). The first is because the SIMPL-1 language does not contain an inverse tangent function, although for this example the angle is small enough to assume $\theta=\tan \theta=\sin \theta$. The second reason is to illustrate the use of a function generator.

\section{FUNCTION GENERATOR}

The tabular values for the function generator are stored beginning with the tag INTAN. The regular calling sequence occurs as needed in calculating the initial condition for $1000 \theta$ (or THEIC). 
The eleven function generators tabular values in INTAN were obtained at $1^{\circ}$ intervals by converting from degrees to radians and multiplying by 1000 . The value of 2292.26 for XMULT and RX were obtained as shown in the remarks section of the FG2\$ description:

$$
\begin{aligned}
\text { XMULT } & =\frac{40000}{17.45}=2292.26 \\
\text { RX } & =12
\end{aligned}
$$

The statement following LRS 12 in the 1 isting is $10^{\prime}$. The apostrophe in $10^{\prime}$ indicates there are 10 (decimal) segments in 11 tabular values.

\section{HEADER MESSAGE}

The method for defining the printed output follows the function gerator storage information on the listing. The tag (or storage location) MSl contains the pseudo-instructions TEXT which causes Teletype printing to occur urtil the symbol a is encountered when the sequence

LAW MS1

JMS TMSG\$

is called by the PRINT subroutine. Notice that subrouting PRINT causes the voltage values of $\mathrm{VA}$ and RHO to be printed beneath the message printed by MSl.

The second header message starting at location MS2 is printed in a similir manner when the sequence

LAW MS 2

JMS TMSG $\$$

is encountered in subroutine PRINT.

\section{ANSWER STORAGE}

The tag sequence

$$
\begin{aligned}
& \text { TBL, } \\
& \text { TBL + } 691^{\prime},
\end{aligned}
$$

saves 600 storage locations for answers, since less than 50 increments of the six ariables were printed out only 300 locations needed to be saved. Th: auto index register 10 (discussed later) is used to store and retrieve the answers. 


\section{USER SUBPROGRAMS AND COMPUTER INITIALIZATION}

Since it was desired to solve the problem in repetitive operation, a delay between compute and I.C. followed by a delay between I.C. and compute was incorporated to dress up the output when plotted on the strip chart recorder. The subroutine DELAY also serves as an example of incorporating a user subroutine within a main program. The subroutine is called by the instruction:

JMS DELAY

subroutine DELAY is composed of the following sequence

$\begin{array}{ll}\text { DELAY, } & \emptyset \\ & \text { LAW } \\ \text { DAC CNT\# } \\ \text { ISZ CNT } \\ \text { JMP } & 2 \\ \text { JMP I DELAY } & \text { NOP } \\ \text { JMP } & -4\end{array}$

The first location in the subroutines calling instruction sequence is used to store the contents of the program counter when entering the subroutine. For this reason it is customarily coded with a zero $(\emptyset)$.

The function of the DELAY is to count from $760000_{8}$ up to 777777 (and then zero) and then return to the main program. The instruction JMP I DELAY provides the mechanism for returning to the main program sequence.

The indirect bit (I) of the JMP I DELAY instruction is used for retrieving the address stored by the program counter in location DELAY when originally entering the subroutine. In other words, the JMP I DELAY instructions looks in storage location DELAY for the address of the next executable statement.

The coding sequence between $B G N 1$ and $B G N$ provides the delay, conversion of I.C. problem variables, and software transfer of the computer to I.C. The coding sequence between the tag BGN and the instruction JMS ICL\$ is used for normal computer initialization which include clearing all flags, initializing the Teletype typing subroutine, selecting the modified instruction set, setting important counters to zero and initializing auto index register 10 for answer storage.

The use of the ICL $\$$ subroutine calling sequence starting with ICL\$ to (but not including) DZM flag\# is used for initializing the real-time clock, selecting the time scale, determining frequency of interrupts, and specifying the subroutines to be serviced during priority interrupts. For this example the time scale is unity and subroutine SERVC is entered every 0.01 sec for answer storage. (Note that since subroutine SERVC is called by the automatic 
interrupt system that the subroutines first storage location does not contain a zero ( $\emptyset$ ) as do subroutines normally called by a main program. Also, note that the last instruction of the subroutine is a JMP RCL\$.)

PRE-CALCULATION OF INITIAL CONDITIONS

Since the computer's mode has been previously set to I.C., the initial conditions are calculated by following the rules of amplitude scaling and building block interconnection as determined by the governing equations. The reader should note that scaling factors for input problem variables properly combine with arithmatic subprogram scale factors to produce the proper output variable scale factor. This is especially true in calculating $1000 \theta$ (or THEIC). However, an addition scale factor of 10 is required in evaluating VIC.

\section{AUTO INDEX REGISTERS FOR ANSWER STORAGE}

The use of auto index registers was mentioned in the sections on Message Printing and Computer Initialization.

Storage locations 10 through 14 are reserved as auto index registers. These locations contain special hardware that increments the contents of the location before executing an instruction. This is why auto index register 10 was initialized with the address of TBL-1.

The first need for using auto index register 10 occurs in storing initial conditions for later retrieval during printout. The instruction sequence

$\begin{array}{lll}\text { DZM I } 10 & \text { (Stores a zero for TIME in 1oca TBL) } \\ \text { DZM I } 10 & \text { (Stores a zero for VDOT in loc TBL+1) } \\ \text { LAC VIC } & \text { (Stores VIC in 1oc TBL+2) } \\ \text { DAC I 10 } & \\ \text { LAC THEIC } & \text { (Stores THEIC in 1oc TBL+3) } \\ \text { DAC I } 10 & \text { (Stores a zero for X in } 10 \mathrm{TBL}+4) \\ \text { DZM I } 10 & \text { (Stores a zero for } \mathrm{Y} \text { in } 10 \mathrm{C} \text { TBL+5) } \\ \text { DZM I } 10 & \end{array}$

The auto index register is not used again until subroutine SERVC is serviced by the automatic priority interrupt system (operating under software control of the ICL $\$$ subroutine). Further utilization of the auto index register is similar to that given above. However, the reader should note that a counter (COUNT1) is used to determine the number of times the PRINT subroutine was entered.

A test to see if computing should terminate immediately preceeds the last instruction of the PRINT subroutine. It is especially important to note that the last instruction of an ICL\$ called service subroutine must be a JMP RCL $\$$. 


\section{THE MAIN COMPUTING LOOP AND SUBROUTINE RINT\$}

The first item of business in the main computing loop is to check the computer mode by calling subroutine MODE. (After the first loop the mode is changed from I.C. to compute.) The second item is to store the last clock value in location NP with the sequence

$$
\begin{array}{ll} 
& \text { JMS } \\
\text { LCCO }, & \emptyset \\
& \text { DAC NP }
\end{array}
$$

This is followed by the calling sequence for subroutine RINT\$. (Note that before evaluating derivatives RINT\$ is called to provide numerical definition of integrator initial conditions.) The derivatives are evaluated by applying amplitude scaling rules and interconnecting mathematical building blocks as shown on the flow diagram.

The integration routine RINT\$ can either operate synchronous or asynchronous with time. Since the CNP $\$$ routine is indirectly serviced by ICL $\$$, the integration in this example is asynchronous with time. Or in other words, fixed time steps are not used. As many time steps (or computing frames) are taken as time permits. By placing a counter in the main loop, 331 steps were evaluated during real-time and only 29 for $x 10$ real-time operation.

(These numbers compare favorably with those evaluated by the equations given in comment 3 of the ICL $\$$ description in Appendix B.)

The test to determine if $Y \geq Y 1$ is done once and is followed by digita1to-analog conversion instructions (a strip chart recorder, oscilloscope, or $X-Y$ plotter is used to display the results). The value of VDOT is divided by 20 before $\mathrm{D} / \mathrm{A}$ conversion by the sequence

LAC VDOT

JMS X.1

LRSS 1

DA4

The two instructions preceding JMP LOOP set a software flag for transferring the computer mode to compute (see subroutine MODE).

\section{TELETYPE PRINTING OF ANSWERS}

The Teletype provides a temporary means of printing out numerical answers (until a line printer is available). However, even with a line printer available, numerical printout is not as desirable as graphical plots already available with the strip chart recorder, oscilloscope, or X-Y plotter.

The first instruction (DPI) of subroutine PRINT disables the priority interrupt. Then if any or all $\mathrm{AC}$ switches are set a numerical printout will 
be obtained when either $x=-60$. or $y=30$. The heading as explained in the Header Message Section is the first item printed. The numerical information stored starting in location $\mathrm{TBL}$ is retrieved by first re-initializing auto index register 10 to $\mathrm{TBL}-1$ with the sequence

LAC $(\mathrm{TBL}-1$

DAC 10

The output is then retrieved by means of an inner and outer nested computing loop. The outer loop initializes COUNT1 to determine when printing is done. The inner loop initializes a second counter for - 5 before printing TIME, VDOT, V; THETA, X, and Y. Each time a 1 ine is printed, the Teletype is given a carriage return before determining if printing is done. If printing has been completed, the Teletype is given a form-feed (to output last page of paper), and control is transferred to DDT for parameter modification or new programming instructions. Note the last instruction of the program is the pseudo instruction PAUSE.

\section{GENERAL REMARKS ABOUT THE PROGRAM}

The program as written required 1840 memory locations (or 23.5\% of the 8192 memory locations) and adequately simulated the problem in real time.* The main loop frame time was approximately $1200 \mathrm{usec}$ (excluding printing). Approximately $200 \mathrm{usec}$ could have been saved by performing the calculation $10^{5} \mathrm{SC}_{\mathrm{d}} \mathrm{p} / 2 \mathrm{M}$ only once in the initialization section and by using an assembly language program for the functions performed by the ICL\$ program.

Memory requirements could have been reduced $7.6 \%$ by using all analog readout methods (such as, $X-Y$ plotter, strip chart recorder, or oscilloscope).

A memory requirements comparison between the SIMPL-1 (a11 digital) and FORTRAN problem solving method is difficult because of our inability to discriminate between systems programs and FORTRAN. However, the FORTRAN assembled 1 isting requires approximately 3.3 times that required by SIMPL-1 (a11 digital) and 6 times that required by SIMPL-1 (hybrid).

\footnotetext{
* The program processed the main loop 331 times during real time operation and 29 times during times ten operation. (Accuracy for times ten operation was not acceptable. The program could theoretically be speeded up by a factor of $\frac{331}{44}$ (or 7.5) and still preserve acceptable accuracy.)
} 
SIMPL-1 ALL OIGITAL SOLUTION FOR THE PILOT EJECTION STUOY (contd)

FORTRAN AND ASSEMALED LISTINGS FOR PILOT EJECTION STUDY

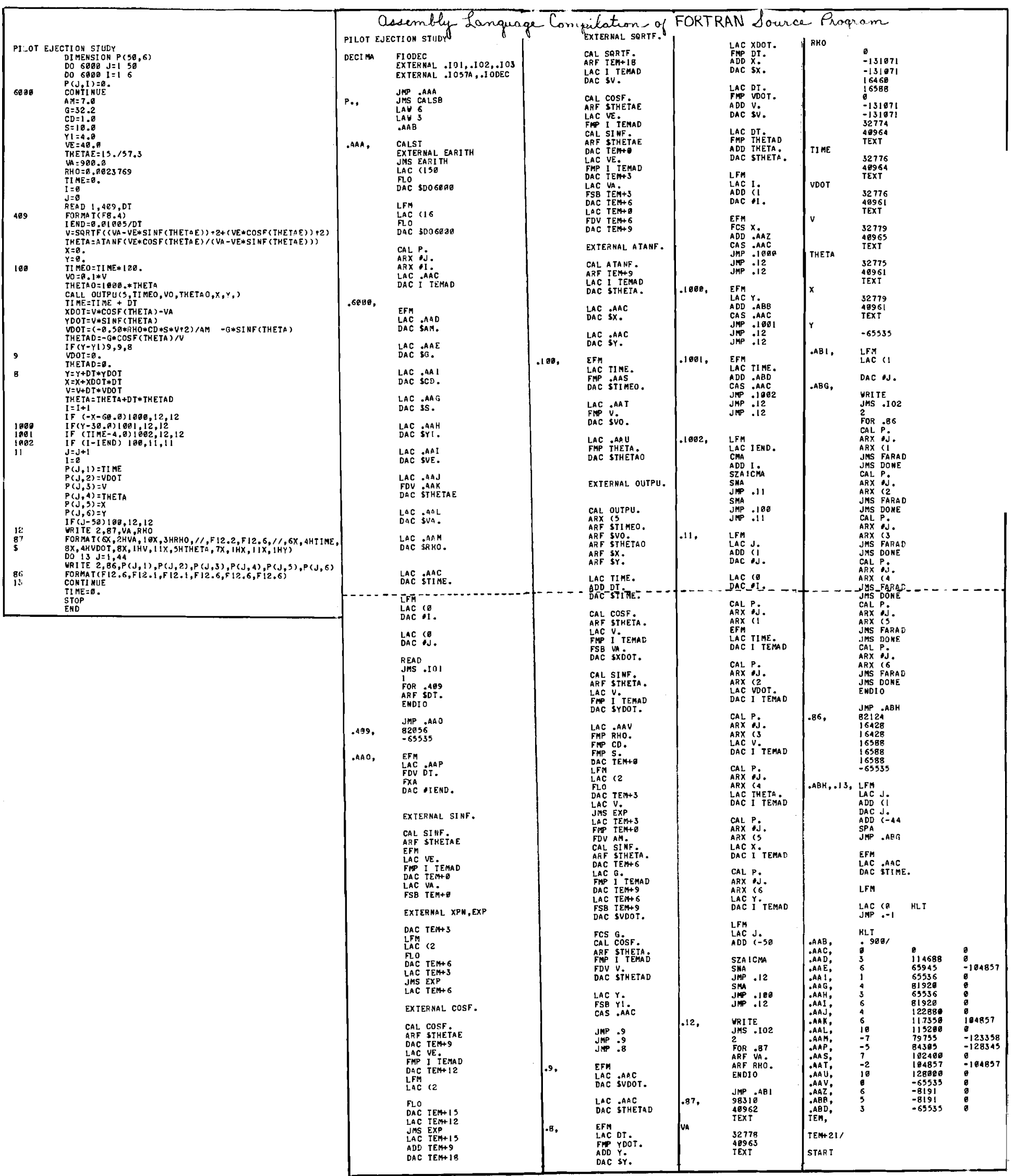


APPENDIX 0

PROCESS CONTROL EXAMPLE ILLUSTRATING ASSEMBLY LANGUAGE PROGRAMMING

OF API CLOCK SERVICE ROUTINE

This example demonstrates che time delay progran with an assembly language clock servtce routine. The ICLS and RCL\$ clock 1 nitiallza tion and clock release programs are not used. (The time required (T)

THE PROBLEM

Assune that it is desired to maintain the vater level at $5 \mathrm{ft} . \pm 0.2 \mathrm{ft}$. In the tank shown $3^{\text {in }}$ the dragram uhȩn the

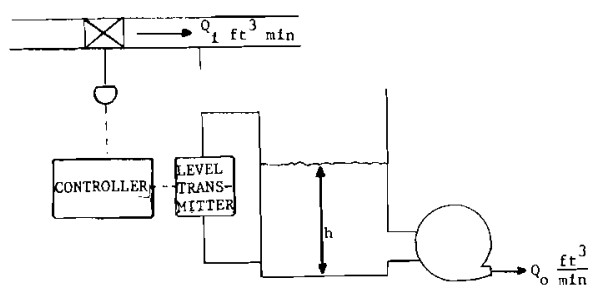

Th1s may be done with a device capable of measuring the level of the tank, a controller, and a valve. A stgnal from the level transmitter is fed to a controller. The controller cona control signal which operates the valve controlling the inle flow.

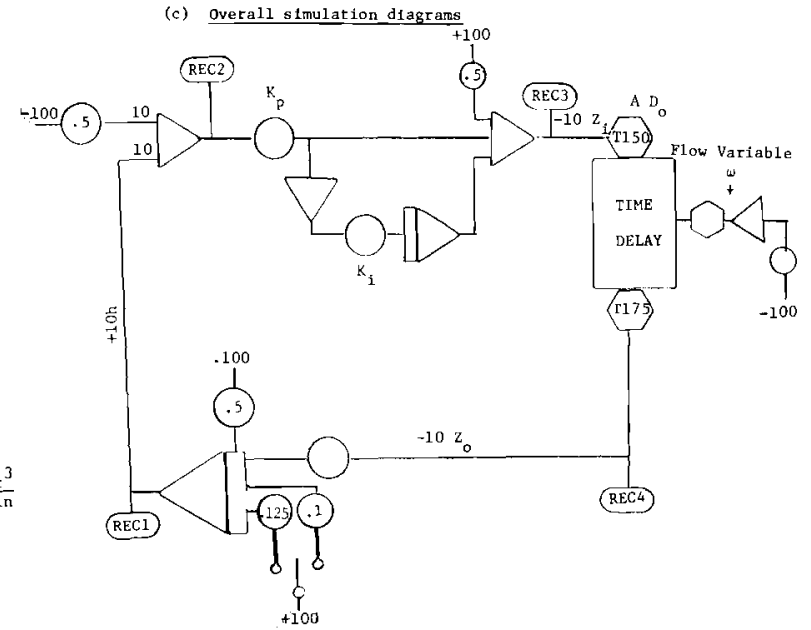

The t1me required for the control system and valve to react to a change in level is usually insigntf tcant. However, for the purpose of this example, suppose that there is a one minute delay for a control systen to someuhet unrealistic, but for th example it allous the opporcunicy of ustrg a time delay.)

To summartze, the equations for this system are shown in the following block diagram.

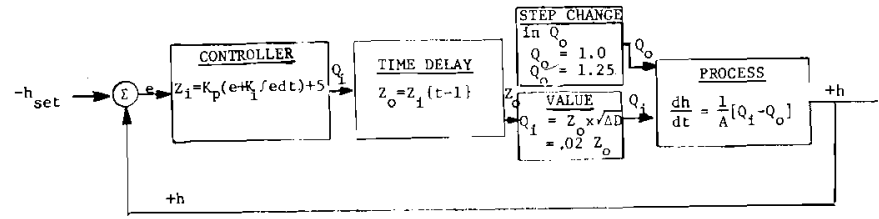

(d) D1gita1 time delay

Before the digital time delay ts used the number of points (N) to be stored and the number of icera-

The value of $\mathrm{N}$ is estimated as explained in Appendix II $\mathrm{N}=\left(\mathrm{R}_{\text {MAX }}\right) \frac{\left.\mathrm{T}^{\mathrm{MAX}}\right)}{\mathrm{ERROR}}$

$=\frac{1}{A}\left(10 Q_{1}-10 Q_{0}\right)\left(\frac{1}{0.25}\right)$

$=1(2.5)\left(\frac{1}{0.25}\right)$

$\approx \underline{\underline{10}}$

$\mathrm{N}_{\mathrm{I}}=\left(\frac{\mathrm{R}_{\text {MAX }}}{\mathrm{ERROR}}\right)\left(\frac{\mathrm{W}_{\text {MIN }}}{W_{\text {MAX }}}\right)+2$

$=(10)\left(\frac{80}{100}\right)+2$

$=10$

Drav the scaled computer diagram for th1s system. Conduct experiments to determine acceptable values of controller gatn (Kp) and the reset race ( $K_{I}$ ) to maintain the tank level at $5 \mathrm{ft} .3 \pm 0.2 \mathrm{ft}$. when the

where,

$$
\begin{aligned}
& A=\text { tank cross sectional area }=1 \mathrm{ft}^{2} \\
& h=\text { level of water 1n tank ( } h_{\text {setpoint }}=5 \mathrm{ft} \text {.) } \\
& q_{f}=\text { inlet flow rate (to be controlled) } \\
& Q_{0}=\text { outlet flow rate (to be varted from } 1.0 \text { to } \\
& \mathrm{e}=\mathrm{h}-\mathrm{h}_{\text {setpotnt }} \\
& =\mathbf{h}-5.0 \\
& \mathrm{~K}_{\mathrm{p}}=\text { gain of proportional controller } \\
& K_{I}=\text { reset rate, repeats per min. }
\end{aligned}
$$

\section{PROBLEM ANALYSIS}

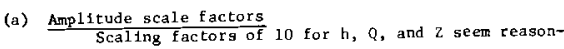
Scaling facto
able because

$$
\begin{aligned}
& 10 h=50 \\
& 10 Q_{0}=10 \text { to } 12.5 \\
& 10 Z_{0}=50
\end{aligned}
$$

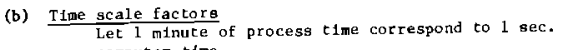

$$
\begin{aligned}
& \text { When the } 5000 \mathrm{cycle} / \mathrm{sec} \text { clock is used } \\
& \begin{aligned}
\text { NCLOCK } & =\frac{5000 \frac{\text { PLSES }}{\text { SEC }}}{N_{I} \frac{\text { ITERTIONS }}{\text { SEC }}} \\
& =\frac{5000}{100} \\
& =500 \frac{\text { PULSES }}{\text { ITERATION }} \text { (Real-Time) } \\
& =50 \text { for ten times real-time }
\end{aligned}
\end{aligned}
$$

(e) Dtgital program \& coding

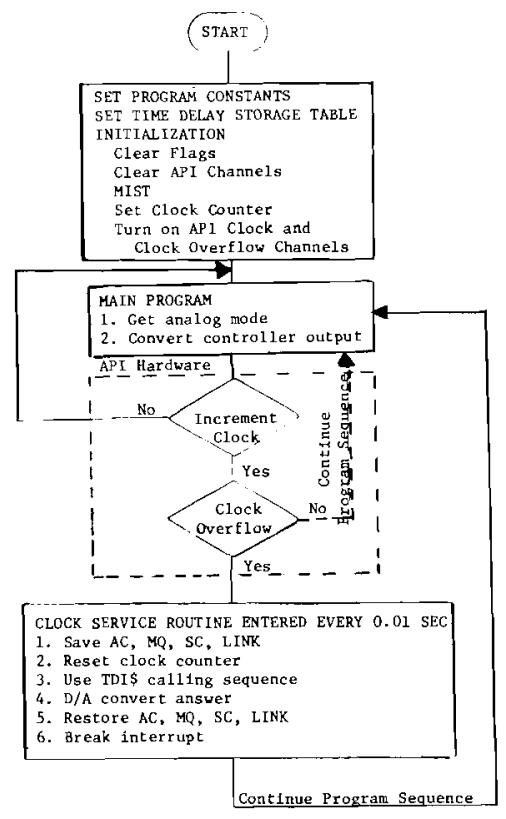




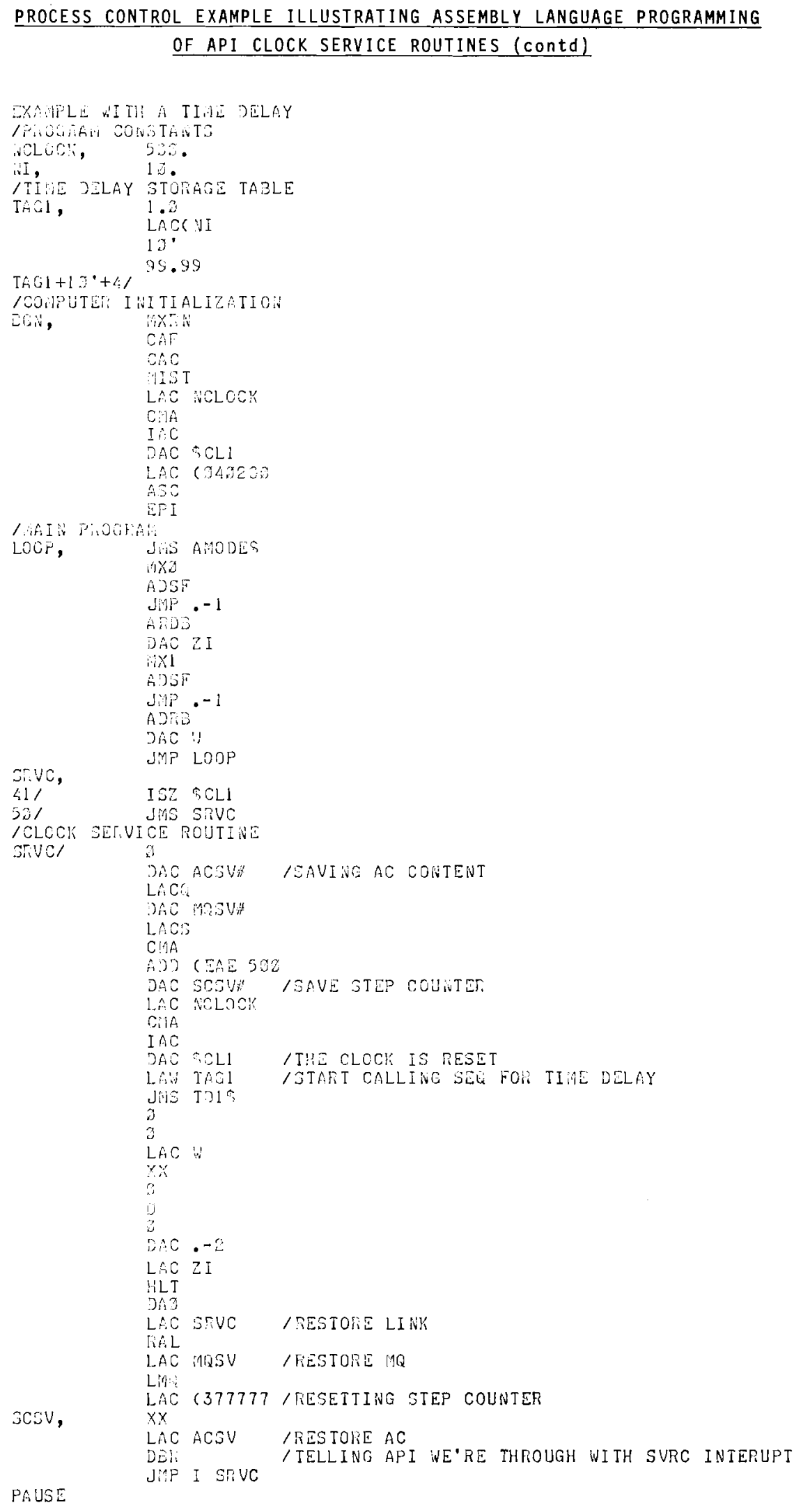


PROCESS CONTROL EXAMPLE ILLUSTRATING ASSEMBLY LANGUAGE PROGRAMMING

OF API CLOCK SERVICE ROUTINES (contd)

The program listing illustration

The program listing illustrates:

1. Program initialization to include:

a. The method of specifying a relocatable assembly and then referring to an absolute address between the main program and clock service rout ine

b. API channels

c. The method for setting API clock counter

2. The main program which includes:

a. Checking the analog mode

b. Assembly language programming of analog to digital conversions (instead of using ADC $\$$ )

3. The API clock service routine which includes:

a. The methods for storing the contents of the LINK, AC, MQ, and step counter after an API interrupt

b. The time delay calling sequence

c. The methods for restoring the contents of the LINK, AC, MQ, and step counter

d. The method of returning to the main program after an interrupt

PROGRAM IN ITIALI ZATION

The first program entry in the listing (other than the title or program comments) specifies a relocatable assembly if it is not an absolute address assignment such as $100 / 5192 /$ etc. For example,

$866 /$

NCLOCK , $\quad 5 \emptyset \emptyset$

would have specified an absolute assembly starting in location 866 (i.e. NCLOCK $=866)$.

Absolute addresses for the API clock and clock overflow channels were used between the main program and subroutine SRVC. The instruction sequence

$\begin{array}{lll}\text { SRVC, } & & \\ 41 / & \text { ISZ } & \text { \$CL1 } \\ 5 \emptyset / & \text { VMS } & \text { SRVS } \\ \text { SRVC } / & \emptyset & \end{array}$

is used to allow subroutine SRVC to be assembled relocatable. If the tag [SRVC,] were eliminated previous to $41 /$ then the starting location of the subroutine SRVC would be location 51. This would create three problems. First, if subroutine SRVC required more space than available between location 
51 and the programs first relocatable address (NCLOCK), the assembler would mask the overlapping portion of the program. The second problem would occur because the linking loader would not load the program unless a large enough relocation constant was specified. Finally, if the program were loaded, the locations between 40 and 57 are reserved as API channel locations. Spurious API signals could cause interrupts which in turn would cause instructions in those locations to be interrupted and their corresponding instructions executed. This in essence would clobber the program and necessitate reloading.

The method for initializing the clock counter is given by the sequence:

$\begin{array}{ll}\text { LAC } & \text { NCLOCK } \\ \text { CMA } & \\ \text { IAC } & \\ \text { DAC } & \$ C L 1\end{array}$

The IAC instruction is required since the incrementing instruction (ISZ \$CL1) stored in location 41 is a two's compliment instruction. As mentioned in Example 1 of Appendix F, control is transferred to the clock overflow channel (loc 51) when the clock overflows from $777777_{8}$ to $\emptyset$. Since the ISZ instruction stored in location 41 operates in two's compliment arithmetic, the number stored in \$CLl must therefore be the negative two's compliment of the synchronizing counter NCLOCK. The IAC instruction following the CMA instructions provides conversion between one's and two's compliment arithmetic. (It should be noted that the same clock initializing sequence is contained in the API clock service routine.)

The multiplexer and API are initialized by the sequence

$$
\begin{aligned}
& \text { MXRN } \\
& \text { CAF } \\
& \text { CAC }
\end{aligned}
$$

\section{MAIN PROGRAM}

Since the time delay subroutine needs the analog computer mode, mode checking is done in the main program with a JMS AMODE $\$$. The computing frame time for this problem is reduced by A/D converting with assembly language rather than with subroutine ADC\$. The remaining MAIN PROGRAM instructions through the instruction JMP LOOP demonstrate assembly language conversion and storage for the delayed quantity $Z I$ and the variable delay quantity $W$. (Note that the multiplexer was initialized with an MXRN in the program initialization section.) 


\section{API CLOCK SERVICE ROUTINE}

As previously mentioned, the program calls for a relocatable assembly. However, the fixed API channel locations 41 and 50 must be referenced. This is done immediately after the main program and before the clock service rou-

tine with the sequence

$\begin{array}{lll}\text { SRVC, } & & \\ 41 / & \text { ISZ } & \text { \$CL } 1 \\ 5 \emptyset / & \text { JMS } & \text { SRVC }\end{array}$

The first order of business for the clock service routine is to save the contents of the LINK, AC, MQ, and the EAE step counter. These registers must be saved since the computer may be in the middle of a "main loop" computation when the clock overflow occurs. The API does not allow the "main loop" program to finish computations before a control is transferred to the service routine. All registers must be restored before control is returned to the main loop. The instruction in location $5 \emptyset$ (JMS SRVC) provides the mechanism for saving the contents of the LINK in bit and program counter in bits 5-17 of memory location SRVC/. The instructions for saving the contents of the AC and MQ are straight forward. However, the instruction sequence for saving and recalling the contents of the EAE step counter involves special microprogramming technique for generating a dummy MORM instruction which restores the SC register. The instruction LACS loads the accumulator with the contents of the step counter. To restore the step counter, a dummy normalization instruction must be created through microprogramming and then executed following the instruction LAC (377777. The following example will illustrate the technique:

If the step counter contained a shift count of 44 it would appear in negative two's compliment arithmetic as

$$
011100
$$

The contents of the accumulator after the instructions

LACS

CMA

are

$\begin{array}{llllll}111 & 111 & 111 & 111 & 100 & 011\end{array}$


The contents of the AC after the instruction*

\section{ADD $\quad$ EAE $5 \emptyset \emptyset$}

is the instruction NORM but with the step count modified to equal the original content of the SC. This modified NORM is stored in location SCSV. (The last two octal numbers in the NORM instruction are the step count.)

The sequence for resetting the \$CLl and the time delay calling sequence follows the instructions for saving the important registers. Immediately following the time delay calling sequence are the instructions for digital to analog conversion and restoring the LINK, MQ, step counter, and AC.

The LINK is restored by merely rotating the contents of location SRVC one position to the right. The instructions for restoring the MQ is equally straight forward. To understand how the step counter is restored one must recall that the NORM (or a modified NORM) instruction shifts the contents of the $\mathrm{AC}$ and MQ until AC bit $\emptyset$ is different than bit 1 or the step count is exceeded. The instruction

\section{LAC $\quad(377777$}

is used to load AC bits $\emptyset$ and 1 with different numbers so that when the modified normalization instruction is executed no shifting occurs. The step count from the NORM instruction is then incremented, complimented, and deposited in the step counter by computer hardware.

Control is returned to the main program by first disabling the automatic priority interrupt with the instruction DBR and then resetting the program counters address with the JMP I SRVC instruction.

* The 5 in the instruction EAE $5 \emptyset \emptyset$ is the micro code for a long right shift. The computer performs the operation $A D D$ (EAE $5 \emptyset \emptyset$ as folzows:

$+$\begin{tabular}{llllll}
111 & 111 & 111 & 111 & 100 & 011 \\
110 & 100 & 000 & 101 & 000 & 000 \\
\hline 110 & 100 & 000 & 100 & 100 & 011 \\
end around carry & & -1 \\
\hline 110 & 100 & 000 & 100 & 100 & 100
\end{tabular}

Contents of $A C$ (Binary code for EAE $5 \emptyset \emptyset$ 
. 
No. of

Copies

OFFSITE

1 Adage, Inc. 1079 Commonwealth Ave. Boston, Mass 02215

L. H. Teitelbaum

1

AEC Chicago Patent Group

G. H. Lee

M Shaw, Director, RDT

Asst Dir for Nuclear Safety

Components $\mathrm{Br}$, RDT:PE

Asst Dir for Reactor Engrg

Core Design $\mathrm{Br}$, RDT:RE (2)

Fuel Fabrication $\mathrm{Br}$, RDT : RE

Fuel Handling $\mathrm{Br}$, RDT: RE

Reactor Vessels Br, RDT:RE

Asst Dir for Reactor Tech

Fuels \& Materia1s Br, RDT:RT

Reactor Physics Br, RDT: RT

AEC Idaho Operations office

Nuclear Technology Division

C. W. Bills, Director

G. Farris

AEC Office of the Controller

Data Processing Evaluation

and Control Branch

Washington, D.C. 20545

John Kresky

James Wagner

AEC Division of Reactor Development and Technology

Analysis \& Evaluation Br, RDT:NS

Environmental \& Sanitary Engrg Br, RDT:NS

Research \& Development Br, RDT:NS

Asst Dir for Plant Engrg, RDT

Applications \& Facilities Br, RDT:PE

Instrumentation \& Control Br, RDT:PE

Systems Engineering $\mathrm{Br}$, RDT: $\mathrm{PE}$

Asst Dir for Program Analysis, RDT

Asst Dir for Project Mgmt, RDT

Liquid Metals Projects Br, RDT:PM

FFTF Project Manager, RDT:PM (3)

Control Mechanisms Br, RDT:RE

Chemistry \& Chemical Separations Br, TDT:RT (2)

Special Technology $\mathrm{Br}$, RDT: RT

AEC Division of Technical Information Extension

AEC Oak Ridge National Laboratory

AEC San Francisco Operations Office

Director, Reactor Division 
No. of

Copies

4

AEC Site Representatives

Argonne National Laboratory

Atomics International

Atomic Power Development Assoc.

General Electric Company

$4 \quad$ Applied Dynamics

P.0. Box 1488

Ann Arbor, Michigan 48106

J. D. Kennedy

R. Smith

R. Morgon

D. Moran

2 Argonne National Laboratory

R. A. Jaross, LMFBR Program Office

F. Morehouse

2

Atomics International

L. E. Glasgow

Liquid Metal Engrg Center

R. W. Dickinson

1

Atomic Power Development Assoc.

B. V. D. Farris

A. G. Hosler

2 Babcock \& Wilcox Co.

Atomic Energy Division

S. H. Esleeck

Boiler Division

T. P. Farre11

1 Boeing Airplane Division

Renton, Washinton

D. Taylor

2

Contro1 Data Corporation

8100-34 Ave. South

Minneapolis, Minn 55420

J. K. Munson

F. J. Sansom

1 Combustion Engineering 1000 MWe Follow-On Study

W. P. Staker, Project Manager

3

Digital Equipment Corporation Maynard, Mass

J. A. Jones

W. Mackenzie

Decus Library 


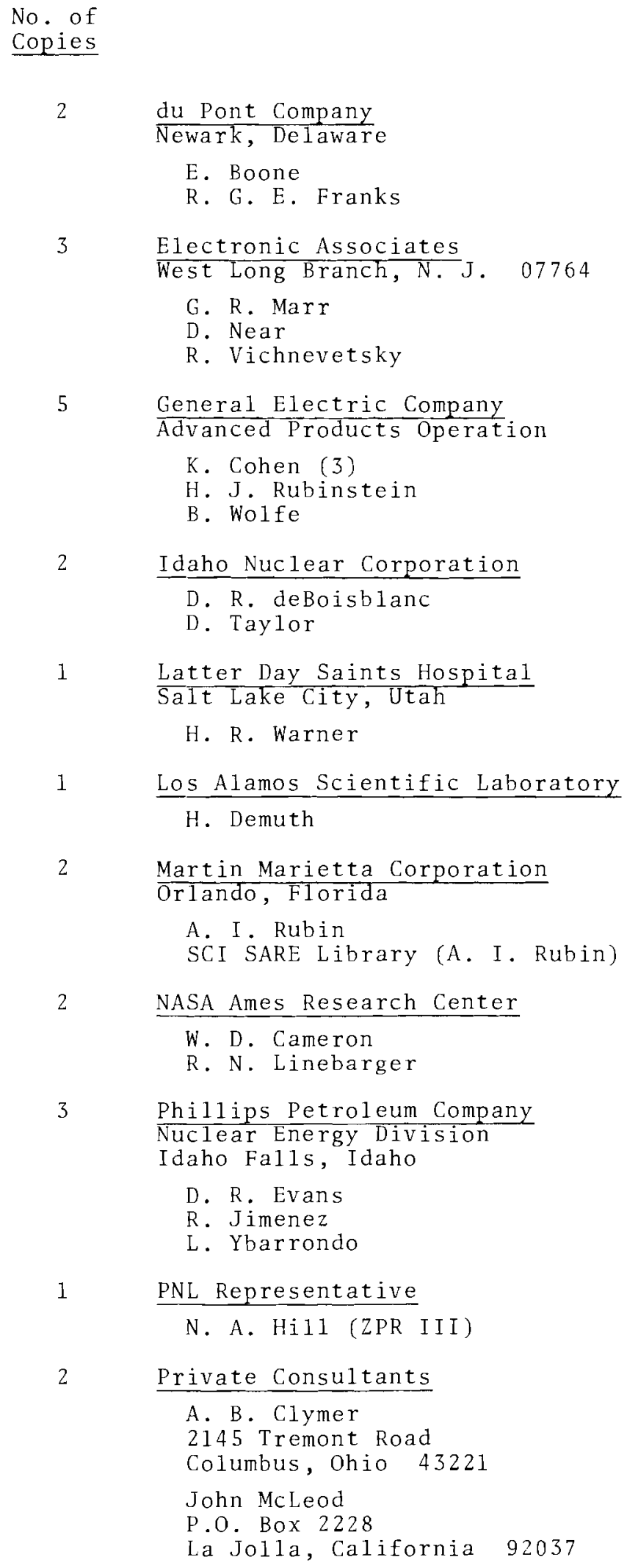

1 PNL Representative

N. A. Hill (ZPR III)

$2 \quad$ Private Consultants

A. B. Clymer

2145 Tremont Road

Columbus, Ohio 43221

John McLeod

P.O. Box 2228

La Jolla, California 92037 
No. of

Copies

2

$$
\frac{\text { Purdue University }}{\text { Lafayette, Indiana }}
$$

B. Kohr

T. J. Williams

1 Scientific Data Systems

1649-17th Street

Santa Monica, California

B. Gold

1

Stanford University

Nuclear Division

Division of Mechanical Engrg

Stanford, California 94305

R. Sher

1

United Aircraft

Hartford, Connecticut

R. Be11urado

1 University of Arizona

Tucson, Arizona

G. Korn

5 University of Idaho

Moscow, Idaho

L. L. Edwards

R. Furgason

G. G. Hespe1t

M. L. Jackson

Tony Rigas

1

University of British Columbia Vancouver, B.C.

Avrom Soudack

1

Washington State University

Pullman, Washinton

Harriet Rigas

5

Westinghouse Electric Corporation

Atomic Power Division

Advanced Reactor Systems

M. Heck

J. C. R. Ke $11 y$

1 Wright Patterson Air Force Base Dayton, Ohio

L. M. Warshawsky 
No. of

Copies

ONSITE-HANFORD

1

AEC Chicago Patent Group

R. K. Sharp (Richland)

4

AEC RDT Site Representatives - PNL

P. G. Holsted (2)

L. R. Lucas

A. D. Toth

10 AEC Richland Operations office

FFTF Project office (2)

D. W. Gossard

H. A. House

F. J. Hughes

J. H. Krema

M. J. Plahuta

C. L. Robinson

D. G. Williams

Technical Information Library

3

Atlantic Richfield Hanford Company

W. L. Godfrey

P. J. Smith

R. A. Watrous

4

Battelle Memorial Institute (Columbus)

M. Tixton (1)

4

Computer Science Corporation

W. Hamilton

J. Orton

J. Peterson

J. Ward

1

Donald W. Douglas Laboratory

R. B. Goranson

6 Douglas United Nuclear

C. L. Goss

P. D. Gross

W. Nechodom

C. F. Poor

R. K. Robinson

Dun File

1

$$
\frac{\text { University of Washington }}{\text { Center for Graduate Study }}
$$

G. Garlick 
No. of Copies

D. T. Aase

G. E. Akre

F. W. Albaugh

W. G. Albert

R. T. Allemann

$\mathrm{J}$. K. Anderson

S. O. Arneson

R. L. Armstrong

F. J. Arrotta

E. R. Astley

J. M. Atwood

R. J. Ausere

R. J. Bashor

J. M. Batch

T. Bauman

T. M. Beet 1 e

R. D. Benham (5)

C. A. Bennett

R. A. Bennett

A. G. Blasewitz

J. R. Boldt

C. L. Boyd

D. C. Boyd

C. L. Brown

W. L. Bunch

R. A. Burnett

K. M. Busness

C. P. Cabel1

A. C. Callen

C. M. Cantre11

J. R. Carrel1

N. E. Carter

W. E. Cawley

W. L. Chase

E. J. Cheyney

T. T. Claudson

E. D. Clayton

P. D. Cohn

C. R. Cole

D. L. Condotta

R. R. Cone

J. H. Cox

V. L. Crow

G. M. Dalen

J. M. Davidson

F. G. Dawson

M. H. Deardorff

D. R. de Halas

J. L. Deichman

V. A. Deliso

D. E. Deonigi

P. J. Dionne

G. E. Driver

R. V. Dulin

$\mathrm{J}$. A. Eaglin

J. F. Erben

M. D. Erickson

E. A. Eschbach

L. M. Finch

C. D. Flowers
H. P. Foote

J. C. Fox

R. C. Free

E. E. Garrett

L. H. Gerhardstein (5)

S. M. Gil1

A. L. Gunby

V. W. Gustafson

J. P. Hale

H. Hardy

D. W. Hartmann

R. A. Harvey

B. R. Hayward

J. W. He $1 \mathrm{~m}$

R. J. Hennig

G. M. Hesson

P. L. Hofmann

R. L. Hooper

G. Jansen, Jr.

R. T. Jaske

B. M. Johnson

H. G. Johnson

E. M. Johnston

J. N. Judy

$\mathrm{J}$. H. Kinginger

C. N. Knudson

J. R. Kosorok

D. D. Lanning

H. D. Lenkersdorfer

W. F. Lenzke (5)

R. C. Liikala

C. W. Lindenmeier

H. E. Little

W. W. Little

J. D. Lodge

R. E. Mahan

W. B. McDonald

W. R. Markillie

M. H. Meuser

R. A. Moen

C. A. Munro

C. R. Nash

D. M. Nero

W. L. Nicholson

C. Oster

H. M. Parker

R. S. Paul

M. G. Patrick

J. A. Perry

R. E. Peterson

A. M. Platt

W. A. Reardon

R. L. Reynolds

R. L. Richardson

W. D. Richmond

W. E. Roake

D. L. Rohde

R. B. Rothrock

J. T. Russell

W. Sale 
Battelle-Northwest (contd)

G. A. Sawyer

J. D. Schaffer

L. C. Schmidt

F. H. Shadel

D. W. Shannon

D. E. Simpson

M. O. Slater

C. R. F. Smith

N. B. Smith

W. G. Spear

R. J. Squires

D. D. Stepnewski

G. H. Strong

C. D. Swanson

G. R. Taylor

C. R. Tipton, Jr.

G. G. Thieme

J. C. Tobin

K. G. Toyoda

F. W. Van Wormer

B. B. Vinson

M. A. Voge 1

G. L. Waldkoetter

R. A. Wa1ker

P. C. Wa 1 kup

D. M. Walley

J. H. Westsik

L. A. Whinery

R. D. Widrig

T. W. Withers

N. G. Wittenbrock

M. R. Wood

F. W. Woodfield

D. C. Worlton

G. A. Worth

FFTF File (2)

Technical Information Files (5)

Technical Publications (2)

Extra (100) 
-

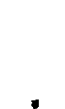

' 